Supplemental Materials

Table for Compound 1: $\left[\mathbf{M g}_{2}(\mathrm{OMes})(\boldsymbol{\mu} \text {-OMes })_{2}(\mathbf{O H})(\text { thf })_{2}\right]_{2} \cdot \mathbf{2 T H F}$ 
Table 1. Crystal data and structure refinement for compound 1.

Identification code

Empirical formula

Formula weight

Crystal system

Space group

Unit cell dimensions

Volume

Z

Density (calculated)

Absorption coefficient

Crystal size

$\mathrm{F}(000)$ wt007

C78 H116 Mg4 O14

1374.95

Monoclinic

$\mathrm{C} 2 / \mathrm{c}$

$\mathrm{a}=24.5045(6) \AA$

$\alpha=90^{\circ}$.

$\mathrm{b}=13.9953(4) \AA$

$\beta=109.8670(10)^{\circ}$.

$\mathrm{c}=24.3557(6) \AA$

$\gamma=90^{\circ}$.

7855.6(4) $\AA^{3}$

4

$1.163 \mathrm{~g} / \mathrm{cm}^{3}$

$0.106 \mathrm{~mm}^{-1}$

$0.50 \times 0.40 \times 0.34 \mathrm{~mm}^{3}$

2976 


\section{Data Collection:}

Diffractometer

Theta range for data collection

Index ranges

Scan Type

Temperature

Wavelength

Reflections collected

Independent reflections

Completeness to theta $=25.00^{\circ}$

Absorption correction

Max. and min. transmission
CCD area detector

1.70 to $25.00^{\circ}$.

$-29<=\mathrm{h}<=29,-16<=\mathrm{k}<=16,-23<=\mathrm{l}<=28$

phi and omega scans

88(2) K

$0.71073 \AA$

23432

$6919[\mathrm{R}(\mathrm{int})=0.0499]$

$99.9 \%$

Semi-empirical from equivalents

0.9648 and 0.9488

direct methods

Full-matrix least-squares on $\mathrm{F}^{2}$

6919 / $47 / 454$

1.054

$\mathrm{R} 1=0.0663, \mathrm{wR} 2=0.1882$

$\mathrm{R} 1=0.1000, \mathrm{wR} 2=0.2113$

0.00061(19)

0.873 and -0.465 e. $\AA^{-3}$ 
Table 2. Atomic coordinates ( $\times 10^{4}$ ) and equivalent isotropic displacement parameters $\left(\AA^{2} \times 10^{3}\right)$ for compound 1 . $U(\mathrm{eq})$ is defined as one third of the trace of the orthogonalized $U^{i j}$ tensor.

\begin{tabular}{|c|c|c|c|c|}
\hline & $\mathrm{x}$ & $\mathrm{y}$ & $\mathrm{z}$ & $\mathrm{U}(\mathrm{eq})$ \\
\hline $\operatorname{Mg}(1)$ & $5326(1)$ & $-3485(1)$ & $1763(1)$ & $21(1)$ \\
\hline $\operatorname{Mg}(2)$ & $5620(1)$ & $-2379(1)$ & 2932(1) & $20(1)$ \\
\hline $\mathrm{O}(1)$ & $5329(1)$ & $-4846(2)$ & $1806(1)$ & $27(1)$ \\
\hline $\mathrm{C}(1)$ & $5171(2)$ & $-5667(2)$ & $1523(1)$ & $26(1)$ \\
\hline$C(2)$ & $4619(2)$ & $-6063(2)$ & $1452(1)$ & $25(1)$ \\
\hline$C(3)$ & $4449(2)$ & $-6915(3)$ & $1141(2)$ & $31(1)$ \\
\hline$C(4)$ & $4812(2)$ & $-7413(3)$ & $907(2)$ & $34(1)$ \\
\hline$C(5)$ & $5361(2)$ & $-7036(3)$ & 1001(2) & $32(1)$ \\
\hline$C(6)$ & $5552(2)$ & $-6179(3)$ & $1307(2)$ & $30(1)$ \\
\hline$C(7)$ & $4244(2)$ & $-5595(3)$ & $1746(2)$ & $29(1)$ \\
\hline $\mathrm{C}(8)$ & $4620(2)$ & $-8330(3)$ & $567(2)$ & $46(1)$ \\
\hline $\mathrm{C}(9)$ & $6159(2)$ & $-5829(3)$ & $1417(2)$ & $35(1)$ \\
\hline $\mathrm{O}(2)$ & $5990(1)$ & $-2802(2)$ & $2357(1)$ & $22(1)$ \\
\hline $\mathrm{C}(10)$ & $6548(1)$ & $-2706(2)$ & $2372(1)$ & $22(1)$ \\
\hline $\mathrm{C}(11)$ & $6925(1)$ & $-3497(2)$ & $2554(1)$ & $26(1)$ \\
\hline$C(12)$ & $7482(2)$ & $-3423(3)$ & $2536(2)$ & $31(1)$ \\
\hline$C(13)$ & $7690(2)$ & $-2593(3)$ & $2363(2)$ & $36(1)$ \\
\hline$C(14)$ & $7319(2)$ & $-1817(3)$ & $2214(2)$ & $33(1)$ \\
\hline$C(15)$ & $6750(2)$ & $-1857(2)$ & $2214(2)$ & $26(1)$ \\
\hline$C(16)$ & $6725(2)$ & $-4375(3)$ & $2780(2)$ & $32(1)$ \\
\hline$C(17)$ & $8300(2)$ & $-2558(3)$ & $2343(2)$ & $52(1)$ \\
\hline $\mathrm{C}(18)$ & $6368(2)$ & $-978(3)$ & $2038(2)$ & $33(1)$ \\
\hline $\mathrm{O}(3)$ & $5442(1)$ & $-2831(2)$ & $3639(1)$ & $23(1)$ \\
\hline$C(19)$ & $5724(2)$ & $-2487(3)$ & 4191(1) & $26(1)$ \\
\hline $\mathrm{C}(20)$ & $6205(2)$ & $-2991(3)$ & $4568(2)$ & $30(1)$ \\
\hline $\mathrm{C}(21)$ & $6494(2)$ & $-2610(3)$ & $5122(2)$ & $38(1)$ \\
\hline $\mathrm{C}(22)$ & $6329(2)$ & $-1771(3)$ & $5318(2)$ & $40(1)$ \\
\hline $\mathrm{C}(23)$ & $5851(2)$ & $-1296(3)$ & $4942(2)$ & $38(1)$ \\
\hline $\mathrm{C}(24)$ & $5540(2)$ & $-1644(3)$ & $4379(1)$ & $28(1)$ \\
\hline $\mathrm{C}(25)$ & $6393(2)$ & $-3914(3)$ & $4383(2)$ & $42(1)$ \\
\hline$C(26)$ & $6654(2)$ & $-1406(4)$ & $5928(2)$ & $62(1)$ \\
\hline
\end{tabular}




\begin{tabular}{|c|c|c|c|c|}
\hline $\mathrm{C}(27)$ & $5007(2)$ & $-1136(3)$ & $3997(2)$ & $36(1)$ \\
\hline $\mathrm{O}(4)$ & 5000 & $-3398(2)$ & 2500 & 19(1) \\
\hline $\mathrm{O}(5)$ & 5000 & $-1518(2)$ & 2500 & $23(1)$ \\
\hline $\mathrm{O}(6)$ & $5623(1)$ & $-3391(2)$ & $1047(1)$ & $28(1)$ \\
\hline $\mathrm{C}(28)$ & $5236(2)$ & $-3755(3)$ & $492(2)$ & $31(1)$ \\
\hline $\mathrm{C}(29)$ & $5628(2)$ & $-4269(3)$ & $223(2)$ & $32(1)$ \\
\hline $\mathrm{C}(30)$ & $6200(2)$ & $-3717(3)$ & $450(2)$ & $37(1)$ \\
\hline $\mathrm{C}(31)$ & $6172(2)$ & $-3159(3)$ & $978(2)$ & $41(1)$ \\
\hline $\mathrm{O}(7)$ & $6300(1)$ & $-1468(2)$ & $3394(1)$ & $26(1)$ \\
\hline$C(32)$ & $6898(2)$ & $-1709(3)$ & $3725(2)$ & $31(1)$ \\
\hline $\mathrm{C}(33)$ & $7238(2)$ & $-801(4)$ & $3748(3)$ & $53(2)$ \\
\hline $\mathrm{C}(34)$ & $6797(2)$ & $-13(3)$ & $3583(3)$ & $48(2)$ \\
\hline $\mathrm{C}(35)$ & $6224(2)$ & $-480(3)$ & $3526(2)$ & $34(1)$ \\
\hline $\mathrm{O}\left(7^{\prime}\right)$ & $6300(1)$ & $-1468(2)$ & $3394(1)$ & $26(1)$ \\
\hline$C\left(32^{\prime}\right)$ & $6898(2)$ & $-1709(3)$ & $3725(2)$ & $31(1)$ \\
\hline $\mathrm{C}\left(33^{\prime}\right)$ & $7154(7)$ & $-928(10)$ & $4150(10)$ & $53(2)$ \\
\hline$C\left(34^{\prime}\right)$ & $6781(6)$ & $-74(7)$ & $3902(12)$ & $48(2)$ \\
\hline$C\left(35^{\prime}\right)$ & $6224(2)$ & $-480(3)$ & $3526(2)$ & $34(1)$ \\
\hline $\mathrm{O}(1 \mathrm{~S})$ & 5000 & $475(2)$ & 2500 & $40(1)$ \\
\hline $\mathrm{C}(1 \mathrm{~S})$ & $4692(2)$ & 1070(3) & $2778(2)$ & $36(1)$ \\
\hline $\mathrm{C}(2 \mathrm{~S})$ & $4710(1)$ & $2062(2)$ & $2544(2)$ & $28(1)$ \\
\hline $\mathrm{O}(2 \mathrm{~S})$ & $2358(5)$ & 2986(8) & $23(5)$ & $49(1)$ \\
\hline $\mathrm{C}(5 \mathrm{~S})$ & $2462(9)$ & $3222(10)$ & $633(6)$ & $49(1)$ \\
\hline $\mathrm{C}(6 \mathrm{~S})$ & $2226(8)$ & $4152(10)$ & $700(6)$ & $49(1)$ \\
\hline $\mathrm{C}(7 \mathrm{~S})$ & $2200(8)$ & $4618(9)$ & $235(7)$ & $49(1)$ \\
\hline $\mathrm{C}(8 \mathrm{~S})$ & $2462(11)$ & $3982(12)$ & $-82(9)$ & $49(1)$ \\
\hline $\mathrm{O}\left(2 \mathrm{~S}^{\prime}\right)$ & 2893(5) & $3551(8)$ & $438(5)$ & $49(1)$ \\
\hline$C\left(5 S^{\prime}\right)$ & $2512(7)$ & $3378(12)$ & $763(6)$ & $49(1)$ \\
\hline$C\left(6 S^{\prime}\right)$ & $2025(6)$ & $4032(12)$ & $613(6)$ & $49(1)$ \\
\hline$C\left(7 S^{\prime}\right)$ & 1947(6) & 4303(13) & $85(7)$ & $49(1)$ \\
\hline $\mathrm{C}\left(8 \mathrm{~S}^{\prime}\right)$ & $2456(10)$ & $3994(17)$ & $-42(8)$ & $49(1)$ \\
\hline
\end{tabular}


Table 3. Bond lengths [̊̊] for compound 1.

\begin{tabular}{|c|c|c|c|}
\hline $\operatorname{Mg}(1)-\mathrm{O}(1)$ & $1.908(3)$ & $C(20)-C(21)$ & $1.398(5)$ \\
\hline $\operatorname{Mg}(1)-\mathrm{O}(2)$ & $2.015(2)$ & $C(20)-C(25)$ & $1.492(5)$ \\
\hline $\mathrm{Mg}(1)-\mathrm{O}(3) \# 1$ & $2.020(2)$ & $C(21)-C(22)$ & $1.380(6)$ \\
\hline $\operatorname{Mg}(1)-\mathrm{O}(6)$ & $2.111(2)$ & $\mathrm{C}(22)-\mathrm{C}(23)$ & $1.387(6)$ \\
\hline $\operatorname{Mg}(1)-\mathrm{O}(4)$ & $2.2016(11)$ & $C(22)-C(26)$ & $1.515(5)$ \\
\hline $\operatorname{Mg}(2)-\mathrm{O}(5)$ & $1.944(2)$ & $C(23)-C(24)$ & $1.409(5)$ \\
\hline $\mathrm{Mg}(2)-\mathrm{O}(2)$ & $1.999(2)$ & $\mathrm{C}(24)-\mathrm{C}(27)$ & $1.501(5)$ \\
\hline $\mathrm{Mg}(2)-\mathrm{O}(3)$ & $2.018(2)$ & $\mathrm{O}(4)-\mathrm{Mg}(2) \# 1$ & $2.089(2)$ \\
\hline $\mathrm{Mg}(2)-\mathrm{O}(4)$ & $2.089(2)$ & $\mathrm{O}(4)-\mathrm{Mg}(1) \# 1$ & $2.2016(11)$ \\
\hline $\operatorname{Mg}(2)-\mathrm{O}(7)$ & $2.096(2)$ & $\mathrm{O}(5)-\mathrm{Mg}(2) \# 1$ & $1.944(2)$ \\
\hline $\mathrm{O}(1)-\mathrm{C}(1)$ & $1.329(4)$ & $\mathrm{O}(6)-\mathrm{C}(31)$ & $1.448(4)$ \\
\hline$C(1)-C(6)$ & $1.414(5)$ & $\mathrm{O}(6)-\mathrm{C}(28)$ & $1.454(4)$ \\
\hline $\mathrm{C}(1)-\mathrm{C}(2)$ & $1.415(5)$ & $C(28)-C(29)$ & $1.517(5)$ \\
\hline $\mathrm{C}(2)-\mathrm{C}(3)$ & $1.397(5)$ & $C(29)-C(30)$ & $1.530(5)$ \\
\hline$C(2)-C(7)$ & $1.495(5)$ & $C(30)-C(31)$ & $1.525(5)$ \\
\hline $\mathrm{C}(3)-\mathrm{C}(4)$ & $1.395(5)$ & $\mathrm{O}(7)-\mathrm{C}(35)$ & $1.446(4)$ \\
\hline$C(4)-C(5)$ & $1.390(6)$ & $\mathrm{O}(7)-\mathrm{C}(32)$ & $1.453(4)$ \\
\hline$C(4)-C(8)$ & $1.514(5)$ & $\mathrm{C}(32)-\mathrm{C}(33)$ & $1.510(5)$ \\
\hline$C(5)-C(6)$ & $1.405(5)$ & $\mathrm{C}(33)-\mathrm{C}(34)$ & $1.499(7)$ \\
\hline $\mathrm{C}(6)-\mathrm{C}(9)$ & $1.502(5)$ & $\mathrm{C}(34)-\mathrm{C}(35)$ & $1.512(5)$ \\
\hline $\mathrm{O}(2)-\mathrm{C}(10)$ & $1.361(4)$ & $\mathrm{C}\left(33^{\prime}\right)-\mathrm{C}\left(34^{\prime}\right)$ & $1.501(11)$ \\
\hline$C(10)-C(15)$ & $1.392(5)$ & $\mathrm{O}(1 \mathrm{~S})-\mathrm{C}(1 \mathrm{~S}) \# 1$ & $1.438(4)$ \\
\hline$C(10)-C(11)$ & $1.413(5)$ & $\mathrm{O}(1 \mathrm{~S})-\mathrm{C}(1 \mathrm{~S})$ & $1.438(4)$ \\
\hline$C(11)-C(12)$ & $1.385(5)$ & $\mathrm{C}(1 \mathrm{~S})-\mathrm{C}(2 \mathrm{~S})$ & $1.508(5)$ \\
\hline$C(11)-C(16)$ & $1.494(5)$ & $\mathrm{C}(2 \mathrm{~S})-\mathrm{C}(2 \mathrm{~S}) \# 1$ & $1.507(7)$ \\
\hline$C(12)-C(13)$ & $1.391(5)$ & $\mathrm{O}(2 \mathrm{~S})-\mathrm{C}(8 \mathrm{~S})$ & $1.455(14)$ \\
\hline$C(13)-C(14)$ & $1.384(5)$ & $\mathrm{O}(2 \mathrm{~S})-\mathrm{C}(5 \mathrm{~S})$ & $1.457(13)$ \\
\hline$C(13)-C(17)$ & $1.512(5)$ & $\mathrm{O}(2 \mathrm{~S})-\mathrm{O}(2 \mathrm{~S}) \# 2$ & $1.55(2)$ \\
\hline$C(14)-C(15)$ & $1.395(5)$ & $\mathrm{C}(5 \mathrm{~S})-\mathrm{C}(6 \mathrm{~S})$ & $1.457(12)$ \\
\hline$C(15)-C(18)$ & $1.515(5)$ & $\mathrm{C}(6 \mathrm{~S})-\mathrm{C}(7 \mathrm{~S})$ & $1.290(14)$ \\
\hline $\mathrm{O}(3)-\mathrm{C}(19)$ & $1.371(4)$ & $\mathrm{C}(7 \mathrm{~S})-\mathrm{C}(8 \mathrm{~S})$ & $1.462(12)$ \\
\hline $\mathrm{O}(3)-\mathrm{Mg}(1) \# 1$ & $2.020(2)$ & $\mathrm{O}\left(2 \mathrm{~S}^{\prime}\right)-\mathrm{C}\left(8 \mathrm{~S}^{\prime}\right)$ & $1.433(13)$ \\
\hline$C(19)-C(24)$ & $1.395(5)$ & $\mathrm{O}\left(2 \mathrm{~S}^{\prime}\right)-\mathrm{C}\left(5 \mathrm{~S}^{\prime}\right)$ & $1.435(13)$ \\
\hline$C(19)-C(20)$ & $1.414(5)$ & $C\left(5 S^{\prime}\right)-C\left(6 S^{\prime}\right)$ & $1.448(12)$ \\
\hline
\end{tabular}


Symmetry transformations used to generate equivalent atoms:

$\# 1-x+1, y,-z+1 / 2 \quad \# 2-x+1 / 2,-y+1 / 2,-z$ 
Table 4. Bond angles $\left[^{\circ}\right]$ for compound 1.

\begin{tabular}{|c|c|c|c|}
\hline $\mathrm{O}(1)-\mathrm{Mg}(1)-\mathrm{O}(2)$ & $116.55(11)$ & $\mathrm{C}(1)-\mathrm{C}(6)-\mathrm{C}(9)$ & $120.5(3)$ \\
\hline $\mathrm{O}(1)-\mathrm{Mg}(1)-\mathrm{O}(3) \# 1$ & $117.67(11)$ & $\mathrm{C}(10)-\mathrm{O}(2)-\mathrm{Mg}(2)$ & $130.02(19)$ \\
\hline $\mathrm{O}(2)-\mathrm{Mg}(1)-\mathrm{O}(3) \# 1$ & 121.71(10) & $\mathrm{C}(10)-\mathrm{O}(2)-\mathrm{Mg}(1)$ & $128.66(19)$ \\
\hline $\mathrm{O}(1)-\mathrm{Mg}(1)-\mathrm{O}(6)$ & $96.45(10)$ & $\mathrm{Mg}(2)-\mathrm{O}(2)-\mathrm{Mg}(1)$ & $101.24(10)$ \\
\hline $\mathrm{O}(2)-\mathrm{Mg}(1)-\mathrm{O}(6)$ & $98.16(10)$ & $\mathrm{O}(2)-\mathrm{C}(10)-\mathrm{C}(15)$ & $121.6(3)$ \\
\hline $\mathrm{O}(3) \# 1-\mathrm{Mg}(1)-\mathrm{O}(6)$ & $95.58(10)$ & $\mathrm{O}(2)-\mathrm{C}(10)-\mathrm{C}(11)$ & $118.3(3)$ \\
\hline $\mathrm{O}(1)-\mathrm{Mg}(1)-\mathrm{O}(4)$ & $90.29(11)$ & $\mathrm{C}(15)-\mathrm{C}(10)-\mathrm{C}(11)$ & $120.0(3)$ \\
\hline $\mathrm{O}(2)-\mathrm{Mg}(1)-\mathrm{O}(4)$ & $79.43(8)$ & $C(12)-C(11)-C(10)$ & $118.5(3)$ \\
\hline $\mathrm{O}(3) \# 1-\mathrm{Mg}(1)-\mathrm{O}(4)$ & $80.51(8)$ & $C(12)-C(11)-C(16)$ & $121.5(3)$ \\
\hline $\mathrm{O}(6)-\mathrm{Mg}(1)-\mathrm{O}(4)$ & $173.22(12)$ & $C(10)-C(11)-C(16)$ & 119.9(3) \\
\hline $\mathrm{O}(5)-\mathrm{Mg}(2)-\mathrm{O}(2)$ & $105.18(8)$ & $C(11)-C(12)-C(13)$ & $122.7(3)$ \\
\hline $\mathrm{O}(5)-\mathrm{Mg}(2)-\mathrm{O}(3)$ & $107.48(8)$ & $\mathrm{C}(14)-\mathrm{C}(13)-\mathrm{C}(12)$ & $117.2(3)$ \\
\hline $\mathrm{O}(2)-\mathrm{Mg}(2)-\mathrm{O}(3)$ & $141.86(11)$ & $\mathrm{C}(14)-\mathrm{C}(13)-\mathrm{C}(17)$ & $122.4(4)$ \\
\hline $\mathrm{O}(5)-\mathrm{Mg}(2)-\mathrm{O}(4)$ & $81.38(11)$ & $\mathrm{C}(12)-\mathrm{C}(13)-\mathrm{C}(17)$ & $120.3(4)$ \\
\hline $\mathrm{O}(2)-\mathrm{Mg}(2)-\mathrm{O}(4)$ & $82.58(7)$ & $C(13)-C(14)-C(15)$ & $122.6(3)$ \\
\hline $\mathrm{O}(3)-\mathrm{Mg}(2)-\mathrm{O}(4)$ & $83.36(7)$ & $C(10)-C(15)-C(14)$ & $118.9(3)$ \\
\hline $\mathrm{O}(5)-\mathrm{Mg}(2)-\mathrm{O}(7)$ & $104.25(11)$ & $\mathrm{C}(10)-\mathrm{C}(15)-\mathrm{C}(18)$ & $121.9(3)$ \\
\hline $\mathrm{O}(2)-\mathrm{Mg}(2)-\mathrm{O}(7)$ & $95.10(10)$ & $C(14)-C(15)-C(18)$ & $119.3(3)$ \\
\hline $\mathrm{O}(3)-\mathrm{Mg}(2)-\mathrm{O}(7)$ & $95.47(10)$ & $\mathrm{C}(19)-\mathrm{O}(3)-\mathrm{Mg}(2)$ & $122.9(2)$ \\
\hline $\mathrm{O}(4)-\mathrm{Mg}(2)-\mathrm{O}(7)$ & $174.33(10)$ & $\mathrm{C}(19)-\mathrm{O}(3)-\mathrm{Mg}(1) \# 1$ & $135.2(2)$ \\
\hline $\mathrm{C}(1)-\mathrm{O}(1)-\mathrm{Mg}(1)$ & $147.2(2)$ & $\operatorname{Mg}(2)-O(3)-M g(1) \# 1$ & $99.42(10)$ \\
\hline $\mathrm{O}(1)-\mathrm{C}(1)-\mathrm{C}(6)$ & $121.3(3)$ & $\mathrm{O}(3)-\mathrm{C}(19)-\mathrm{C}(24)$ & $120.5(3)$ \\
\hline $\mathrm{O}(1)-\mathrm{C}(1)-\mathrm{C}(2)$ & $120.0(3)$ & $\mathrm{O}(3)-\mathrm{C}(19)-\mathrm{C}(20)$ & $119.6(3)$ \\
\hline$C(6)-C(1)-C(2)$ & $118.6(3)$ & $C(24)-C(19)-C(20)$ & $119.9(3)$ \\
\hline$C(3)-C(2)-C(1)$ & $119.9(3)$ & $\mathrm{C}(21)-\mathrm{C}(20)-\mathrm{C}(19)$ & $118.4(4)$ \\
\hline$C(3)-C(2)-C(7)$ & $120.9(3)$ & $\mathrm{C}(21)-\mathrm{C}(20)-\mathrm{C}(25)$ & $121.0(3)$ \\
\hline$C(1)-C(2)-C(7)$ & 119.1(3) & $\mathrm{C}(19)-\mathrm{C}(20)-\mathrm{C}(25)$ & $120.6(3)$ \\
\hline$C(4)-C(3)-C(2)$ & $122.2(4)$ & $\mathrm{C}(22)-\mathrm{C}(21)-\mathrm{C}(20)$ & $123.1(4)$ \\
\hline$C(5)-C(4)-C(3)$ & $117.4(3)$ & $\mathrm{C}(21)-\mathrm{C}(22)-\mathrm{C}(23)$ & $117.4(3)$ \\
\hline$C(5)-C(4)-C(8)$ & $121.0(4)$ & $\mathrm{C}(21)-\mathrm{C}(22)-\mathrm{C}(26)$ & $120.5(4)$ \\
\hline $\mathrm{C}(3)-\mathrm{C}(4)-\mathrm{C}(8)$ & $121.6(4)$ & $\mathrm{C}(23)-\mathrm{C}(22)-\mathrm{C}(26)$ & $122.1(4)$ \\
\hline$C(4)-C(5)-C(6)$ & $122.6(3)$ & $\mathrm{C}(22)-\mathrm{C}(23)-\mathrm{C}(24)$ & $122.3(4)$ \\
\hline$C(5)-C(6)-C(1)$ & 119.2(3) & $\mathrm{C}(19)-\mathrm{C}(24)-\mathrm{C}(23)$ & $118.9(3)$ \\
\hline$C(5)-C(6)-C(9)$ & $120.2(3)$ & $\mathrm{C}(19)-\mathrm{C}(24)-\mathrm{C}(27)$ & $120.5(3)$ \\
\hline
\end{tabular}




$\begin{array}{lllc}\mathrm{C}(23)-\mathrm{C}(24)-\mathrm{C}(27) & 120.6(3) & \mathrm{Mg}(2)-\mathrm{O}(4)-\mathrm{Mg}(2) \# 1 & 93.83(14) \\ \mathrm{Mg}(2)-\mathrm{O}(4)-\mathrm{Mg}(1) & 92.58(7) & \mathrm{C}(34)-\mathrm{C}(33)-\mathrm{C}(32) & 105.8(3) \\ \mathrm{Mg}(2) \# 1-\mathrm{O}(4)-\mathrm{Mg}(1) & 91.71(7) & \mathrm{C}(33)-\mathrm{C}(34)-\mathrm{C}(35) & 105.5(3) \\ \mathrm{Mg}(2)-\mathrm{O}(4)-\mathrm{Mg}(1) \# 1 & 91.71(7) & \mathrm{O}(7)-\mathrm{C}(35)-\mathrm{C}(34) & 104.4(3) \\ \mathrm{Mg}(2) \# 1-\mathrm{O}(4)-\mathrm{Mg}(1) \# 1 & 92.58(7) & \mathrm{C}(1 \mathrm{~S}) \# 1-\mathrm{O}(1 \mathrm{~S})-\mathrm{C}(1 \mathrm{~S}) & 109.3(4) \\ \mathrm{Mg}(1)-\mathrm{O}(4)-\mathrm{Mg}(1) \# 1 & 173.72(17) & \mathrm{O}(1 \mathrm{~S})-\mathrm{C}(1 \mathrm{~S})-\mathrm{C}(2 \mathrm{~S}) & 105.5(3) \\ \mathrm{Mg}(2)-\mathrm{O}(5)-\mathrm{Mg}(2) \# 1 & 103.42(16) & \mathrm{C}(2 \mathrm{~S}) \# 1-\mathrm{C}(2 \mathrm{~S})-\mathrm{C}(1 \mathrm{~S}) & 101.7(2) \\ \mathrm{C}(31)-\mathrm{O}(6)-\mathrm{C}(28) & 108.1(3) & \mathrm{C}(8 \mathrm{~S})-\mathrm{O}(2 \mathrm{~S})-\mathrm{C}(5 \mathrm{~S}) & 89.0(11) \\ \mathrm{C}(31)-\mathrm{O}(6)-\mathrm{Mg}(1) & 134.9(2) & \mathrm{C}(8 \mathrm{~S})-\mathrm{O}(2 \mathrm{~S})-\mathrm{O}(2 \mathrm{~S}) \# 2 & 136.0(12) \\ \mathrm{C}(28)-\mathrm{O}(6)-\mathrm{Mg}(1) & 116.4(2) & \mathrm{C}(5 \mathrm{~S})-\mathrm{O}(2 \mathrm{~S})-\mathrm{O}(2 \mathrm{~S}) \# 2 & 110.2(11) \\ \mathrm{O}(6)-\mathrm{C}(28)-\mathrm{C}(29) & 104.9(3) & \mathrm{C}(6 \mathrm{~S})-\mathrm{C}(5 \mathrm{~S})-\mathrm{O}(2 \mathrm{~S}) & 112.2(8) \\ \mathrm{C}(28)-\mathrm{C}(29)-\mathrm{C}(30) & 104.0(3) & \mathrm{C}(7 \mathrm{~S})-\mathrm{C}(6 \mathrm{~S})-\mathrm{C}(5 \mathrm{~S}) & 104.8(6) \\ \mathrm{C}(31)-\mathrm{C}(30)-\mathrm{C}(29) & 105.4(3) & \mathrm{C}(6 \mathrm{~S})-\mathrm{C}(7 \mathrm{~S})-\mathrm{C}(8 \mathrm{~S}) & 104.9(6) \\ \mathrm{O}(6)-\mathrm{C}(31)-\mathrm{C}(30) & 107.0(3) & \mathrm{O}(2 \mathrm{~S})-\mathrm{C}(8 \mathrm{~S})-\mathrm{C}(7 \mathrm{~S}) & 110.9(9) \\ \mathrm{C}(35)-\mathrm{O}(7)-\mathrm{C}(32) & 106.3(3) & \mathrm{C}\left(8 \mathrm{~S}^{\prime}\right)-\mathrm{O}\left(2 \mathrm{~S}^{\prime}\right)-\mathrm{C}\left(5 \mathrm{~S}^{\prime}\right) & 94.4(10) \\ \mathrm{C}(35)-\mathrm{O}(7)-\mathrm{Mg}(2) & 124.23(19) & \mathrm{O}\left(2 \mathrm{~S}^{\prime}\right)-\mathrm{C}\left(5 \mathrm{~S}^{\prime}\right)-\mathrm{C}\left(6 \mathrm{~S}^{\prime}\right) & 113.0(8) \\ \mathrm{C}(32)-\mathrm{O}(7)-\mathrm{Mg}(2) & 128.7(2) & \mathrm{C}\left(7 \mathrm{~S}^{\prime}\right)-\mathrm{C}\left(6 \mathrm{~S}^{\prime}\right)-\mathrm{C}\left(5 \mathrm{~S}^{\prime}\right) & 105.5(7) \\ \mathrm{O}(7)-\mathrm{C}(32)-\mathrm{C}(33) & 105.4(3) & \mathrm{C}\left(6 \mathrm{~S}^{\prime}\right)-\mathrm{C}\left(7 \mathrm{~S}^{\prime}\right)-\mathrm{C}\left(8 \mathrm{~S}^{\prime}\right) & 106.2(6) \\ \mathrm{O}\left(2 \mathrm{~S}^{\prime}\right)-\mathrm{C}\left(8 \mathrm{~S}^{\prime}\right)-\mathrm{C}\left(7 \mathrm{~S}^{\prime}\right) & 114.4(8) & & \\ & & & \end{array}$

Symmetry transformations used to generate equivalent atoms:

$\# 1-\mathrm{x}+1, \mathrm{y},-\mathrm{z}+1 / 2 \quad \# 2-\mathrm{x}+1 / 2,-\mathrm{y}+1 / 2,-\mathrm{z}$ 
Table 5. Anisotropic displacement parameters $\left(\AA^{2} \times 10^{3}\right)$ for compound 1. The anisotropic displacement factor exponent takes the form: $-2 \pi^{2}\left[h^{2} a^{* 2} U^{11}+\ldots+2 h k a^{*} b^{*} U^{12}\right]$

\begin{tabular}{|c|c|c|c|c|c|c|}
\hline & $\mathrm{U}^{11}$ & $\mathrm{U}^{22}$ & $\mathrm{U}^{33}$ & $\mathrm{U}^{23}$ & $\mathrm{U}^{13}$ & $\mathrm{U}^{12}$ \\
\hline $\operatorname{Mg}(1)$ & $23(1)$ & $20(1)$ & $19(1)$ & $-1(1)$ & $6(1)$ & $-1(1)$ \\
\hline $\operatorname{Mg}(2)$ & $20(1)$ & $20(1)$ & $17(1)$ & $0(1)$ & $3(1)$ & $0(1)$ \\
\hline $\mathrm{O}(1)$ & $34(1)$ & $22(1)$ & $24(1)$ & $-1(1)$ & $8(1)$ & $-1(1)$ \\
\hline $\mathrm{C}(1)$ & $41(2)$ & $19(2)$ & $16(2)$ & $2(1)$ & $8(2)$ & $2(2)$ \\
\hline$C(2)$ & $33(2)$ & $20(2)$ & $22(2)$ & $2(1)$ & $7(2)$ & $1(1)$ \\
\hline$C(3)$ & $42(2)$ & $28(2)$ & $23(2)$ & $0(2)$ & 1_(2) & $-5(2)$ \\
\hline$C(4)$ & $55(3)$ & $22(2)$ & $24(2)$ & $-2(2)$ & $12(2)$ & $0(2)$ \\
\hline$C(5)$ & $49(2)$ & $26(2)$ & $25(2)$ & $1(2)$ & $16(2)$ & $9(2)$ \\
\hline$C(6)$ & $43(2)$ & $24(2)$ & $24(2)$ & $2(2)$ & $12(2)$ & $3(2)$ \\
\hline$C(7)$ & $32(2)$ & $27(2)$ & $27(2)$ & $-2(2)$ & $8(2)$ & $-4(2)$ \\
\hline$C(8)$ & $74(3)$ & $29(2)$ & $32(2)$ & $-7(2)$ & $15(2)$ & $0(2)$ \\
\hline$C(9)$ & $38(2)$ & $38(2)$ & $31(2)$ & $3(2)$ & $14(2)$ & $7(2)$ \\
\hline $\mathrm{O}(2)$ & $19(1)$ & $22(1)$ & $24(1)$ & $-3(1)$ & $7(1)$ & $-1(1)$ \\
\hline$C(10)$ & $20(2)$ & $25(2)$ & $20(2)$ & $-5(1)$ & $4(1)$ & $-2(1)$ \\
\hline $\mathrm{C}(11)$ & $25(2)$ & $28(2)$ & $21(2)$ & $-4(1)$ & $4(1)$ & $1(1)$ \\
\hline$C(12)$ & $22(2)$ & $35(2)$ & $32(2)$ & $-5(2)$ & $4(2)$ & $6(2)$ \\
\hline $\mathrm{C}(13)$ & $24(2)$ & $42(2)$ & $42(2)$ & $-10(2)$ & $13(2)$ & $-6(2)$ \\
\hline$C(14)$ & $32(2)$ & $31(2)$ & $37(2)$ & $-6(2)$ & $14(2)$ & -1_(2) \\
\hline$C(15)$ & $26(2)$ & $24(2)$ & $26(2)$ & $-3(1)$ & $6(2)$ & $-2(1)$ \\
\hline$C(16)$ & $34(2)$ & $26(2)$ & $33(2)$ & $4(2)$ & $6(2)$ & $6(2)$ \\
\hline$C(17)$ & $31(2)$ & $55(3)$ & $75(3)$ & $-16(2)$ & $23(2)$ & $-7(2)$ \\
\hline $\mathrm{C}(18)$ & $33(2)$ & $25(2)$ & $38(2)$ & $2(2)$ & $7(2)$ & $-4(2)$ \\
\hline $\mathrm{O}(3)$ & $26(1)$ & $25(1)$ & $14(1)$ & $-1(1)$ & $3(1)$ & $-3(1)$ \\
\hline$C(19)$ & $28(2)$ & $29(2)$ & $15(2)$ & $0(1)$ & $3(1)$ & $-8(2)$ \\
\hline$C(20)$ & $30(2)$ & $36(2)$ & $21(2)$ & $3(2)$ & $3(2)$ & $-5(2)$ \\
\hline$C(21)$ & $34(2)$ & $51(3)$ & $22(2)$ & $5(2)$ & $-1(2)$ & $-5(2)$ \\
\hline$C(22)$ & $46(3)$ & $51(3)$ & $20(2)$ & $-3(2)$ & $6(2)$ & $-15(2)$ \\
\hline$C(23)$ & $51(3)$ & $34(2)$ & $28(2)$ & -1_(2) & $14(2)$ & $-14(2)$ \\
\hline$C(24)$ & $32(2)$ & $28(2)$ & $23(2)$ & $0(2)$ & $6(2)$ & $-7(2)$ \\
\hline$C(25)$ & $40(2)$ & $49(3)$ & $29(2)$ & $6(2)$ & $2(2)$ & $16(2)$ \\
\hline$C(26)$ & $75(4)$ & $73(4)$ & $26(2)$ & $-8(2)$ & $1(2)$ & $-26(3)$ \\
\hline
\end{tabular}




\begin{tabular}{llllccc}
$\mathrm{C}(27)$ & $42(2)$ & $26(2)$ & $38(2)$ & $-5(2)$ & $14(2)$ & $1(2)$ \\
$\mathrm{O}(4)$ & $21(2)$ & $15(2)$ & $19(2)$ & 0 & $4(1)$ & 0 \\
$\mathrm{O}(5)$ & $25(2)$ & $18(2)$ & $22(2)$ & 0 & $3(1)$ & 0 \\
$\mathrm{O}(6)$ & $35(1)$ & $31(1)$ & $21(1)$ & $-3(1)$ & $12(1)$ & $-6(1)$ \\
$\mathrm{C}(28)$ & $35(2)$ & $37(2)$ & $20(2)$ & $-4(2)$ & $7(2)$ & $-4(2)$ \\
$\mathrm{C}(29)$ & $41(2)$ & $30(2)$ & $22(2)$ & $-1(2)$ & $9(2)$ & $1(2)$ \\
$\mathrm{C}(30)$ & $42(2)$ & $44(2)$ & $31(2)$ & $-4(2)$ & $20(2)$ & $-5(2)$ \\
$\mathrm{C}(31)$ & $39(2)$ & $52(3)$ & $36(2)$ & $-10(2)$ & $18(2)$ & $-15(2)$ \\
$\mathrm{O}(7)$ & $25(1)$ & $24(1)$ & $22(1)$ & $-2(1)$ & $1(1)$ & $-2(1)$ \\
$\mathrm{C}(32)$ & $22(2)$ & $39(2)$ & $25(2)$ & $-3(2)$ & $-3(2)$ & $0(2)$ \\
$\mathrm{C}(33)$ & $30(3)$ & $50(3)$ & $67(4)$ & $3(3)$ & $0(3)$ & $-11(2)$ \\
$\mathrm{C}(34)$ & $45(3)$ & $40(3)$ & $46(4)$ & $1(2)$ & $0(3)$ & $-13(2)$ \\
$\mathrm{C}(35)$ & $42(2)$ & $27(2)$ & $31(2)$ & $-5(2)$ & $9(2)$ & $-4(2)$ \\
$\mathrm{O}\left(7^{\prime}\right)$ & $25(1)$ & $24(1)$ & $22(1)$ & $-2(1)$ & $1(1)$ & $-2(1)$ \\
$\mathrm{C}\left(32^{\prime}\right)$ & $22(2)$ & $39(2)$ & $25(2)$ & $-3(2)$ & $-3(2)$ & $0(2)$ \\
$\mathrm{C}\left(33^{\prime}\right)$ & $30(3)$ & $50(3)$ & $67(4)$ & $3(3)$ & $0(3)$ & $-11(2)$ \\
$\mathrm{C}\left(34^{\prime}\right)$ & $45(3)$ & $40(3)$ & $46(4)$ & $1(2)$ & $0(3)$ & $-13(2)$ \\
$\mathrm{C}\left(35^{\prime}\right)$ & $42(2)$ & $27(2)$ & $31(2)$ & $-5(2)$ & $9(2)$ & $-4(2)$ \\
$\mathrm{O}(1 \mathrm{~S})$ & $45(2)$ & $17(2)$ & $56(2)$ & 0 & $15(2)$ & 0 \\
$\mathrm{C}(1 \mathrm{~S})$ & $44(2)$ & $27(2)$ & $34(2)$ & $2(2)$ & $12(2)$ & $-5(2)$ \\
$\mathrm{C}(2 \mathrm{~S})$ & $25(2)$ & $25(2)$ & $33(2)$ & $-2(2)$ & $7(2)$ & $-2(1)$ \\
& & & & & & \\
\hline
\end{tabular}


Table 6. Hydrogen coordinates ( $\left.x 1^{4}\right)$ and isotropic displacement parameters $\left(\AA^{2} \times 10^{3}\right)$ for compound 1 .

\begin{tabular}{|c|c|c|c|c|}
\hline & $\mathrm{x}$ & $\mathrm{y}$ & $\mathrm{z}$ & $\mathrm{U}(\mathrm{eq})$ \\
\hline $\mathrm{H}(3 \mathrm{~A})$ & 4073 & -7163 & 1087 & 38 \\
\hline $\mathrm{H}(5 \mathrm{~A})$ & 5618 & -7370 & 852 & 39 \\
\hline $\mathrm{H}(7 \mathrm{~A})$ & 3880 & -5951 & 1657 & 44 \\
\hline $\mathrm{H}(7 \mathrm{~B})$ & 4161 & -4937 & 1605 & 44 \\
\hline $\mathrm{H}(7 \mathrm{C})$ & 4446 & -5591 & 2169 & 44 \\
\hline $\mathrm{H}(8 \mathrm{~A})$ & 4222 & -8481 & 543 & 69 \\
\hline $\mathrm{H}(8 \mathrm{~B})$ & 4879 & -8852 & 765 & 69 \\
\hline $\mathrm{H}(8 \mathrm{C})$ & 4635 & -8252 & 173 & 69 \\
\hline $\mathrm{H}(9 \mathrm{~A})$ & 6359 & -6266 & 1234 & 52 \\
\hline $\mathrm{H}(9 \mathrm{~B})$ & 6369 & -5804 & 1838 & 52 \\
\hline $\mathrm{H}(9 \mathrm{C})$ & 6146 & -5189 & 1250 & 52 \\
\hline $\mathrm{H}(12 \mathrm{~A})$ & 7733 & -3960 & 2647 & 37 \\
\hline $\mathrm{H}(14 \mathrm{~A})$ & 7457 & -1235 & 2109 & 39 \\
\hline $\mathrm{H}(16 \mathrm{~A})$ & 7038 & -4849 & 2889 & 48 \\
\hline $\mathrm{H}(16 \mathrm{~B})$ & 6385 & -4640 & 2476 & 48 \\
\hline $\mathrm{H}(16 \mathrm{C})$ & 6622 & -4210 & 3123 & 48 \\
\hline $\mathrm{H}(17 \mathrm{~A})$ & 8374 & -1924 & 2211 & 78 \\
\hline $\mathrm{H}(17 \mathrm{~B})$ & 8343 & -3044 & 2070 & 78 \\
\hline $\mathrm{H}(17 \mathrm{C})$ & 8579 & -2683 & 2733 & 78 \\
\hline $\mathrm{H}(18 \mathrm{~A})$ & 6589 & -456 & 1945 & 50 \\
\hline $\mathrm{H}(18 \mathrm{~B})$ & 6239 & -785 & 2361 & 50 \\
\hline $\mathrm{H}(18 \mathrm{C})$ & 6029 & -1125 & 1695 & 50 \\
\hline $\mathrm{H}(21 \mathrm{~A})$ & 6820 & -2945 & 5375 & 46 \\
\hline $\mathrm{H}(23 \mathrm{~A})$ & 5729 & -717 & 5067 & 45 \\
\hline $\mathrm{H}(25 \mathrm{~A})$ & 6142 & -4066 & 3985 & 62 \\
\hline $\mathrm{H}(25 \mathrm{~B})$ & 6366 & -4423 & 4648 & 62 \\
\hline $\mathrm{H}(25 \mathrm{C})$ & 6796 & -3858 & 4394 & 62 \\
\hline $\mathrm{H}(26 \mathrm{~A})$ & 6976 & -1839 & 6122 & 94 \\
\hline $\mathrm{H}(26 \mathrm{~B})$ & 6390 & -1376 & 6152 & 94 \\
\hline $\mathrm{H}(26 \mathrm{C})$ & 6807 & -766 & 5903 & 94 \\
\hline
\end{tabular}




\begin{tabular}{|c|c|c|c|c|}
\hline $\mathrm{H}(27 \mathrm{~A})$ & 4943 & -562 & 4197 & 53 \\
\hline $\mathrm{H}(27 \mathrm{~B})$ & 4671 & -1561 & 3914 & 53 \\
\hline $\mathrm{H}(27 \mathrm{C})$ & 5060 & -952 & 3630 & 53 \\
\hline $\mathrm{H}(4)$ & 5000 & $-3950(30)$ & 2500 & 23 \\
\hline $\mathrm{H}(5)$ & 5000 & $-970(30)$ & 2500 & 27 \\
\hline $\mathrm{H}(28 \mathrm{~A})$ & 5027 & -3225 & 238 & 38 \\
\hline $\mathrm{H}(28 \mathrm{~B})$ & 4948 & -4200 & 554 & 38 \\
\hline $\mathrm{H}(29 \mathrm{~A})$ & 5465 & -4247 & -208 & 38 \\
\hline $\mathrm{H}(29 \mathrm{~B})$ & 5684 & -4945 & 351 & 38 \\
\hline $\mathrm{H}(30 \mathrm{~A})$ & 6534 & -4162 & 565 & 45 \\
\hline $\mathrm{H}(30 \mathrm{~B})$ & 6239 & -3277 & 147 & 45 \\
\hline $\mathrm{H}(31 \mathrm{~A})$ & 6499 & -3343 & 1332 & 49 \\
\hline $\mathrm{H}(31 \mathrm{~B})$ & 6197 & -2464 & 914 & 49 \\
\hline $\mathrm{H}(32 \mathrm{~A})$ & 7039 & -2224 & 3528 & 38 \\
\hline $\mathrm{H}(32 \mathrm{~B})$ & 6935 & -1924 & 4123 & 38 \\
\hline $\mathrm{H}(33 \mathrm{~A})$ & 7518 & -696 & 4146 & 64 \\
\hline $\mathrm{H}(33 \mathrm{~B})$ & 7453 & -832 & 3471 & 64 \\
\hline $\mathrm{H}(34 \mathrm{~A})$ & 6782 & 282 & 3208 & 57 \\
\hline $\mathrm{H}(34 \mathrm{~B})$ & 6893 & 488 & 3888 & 57 \\
\hline $\mathrm{H}(35 \mathrm{~A})$ & 6140 & -426 & 3895 & 41 \\
\hline $\mathrm{H}(35 \mathrm{~B})$ & 5903 & -182 & 3207 & 41 \\
\hline $\mathrm{H}(32 \mathrm{C})$ & 7122 & -1786 & 3457 & 38 \\
\hline$H(32 D)$ & 6914 & -2319 & 3935 & 38 \\
\hline $\mathrm{H}(33 \mathrm{C})$ & 7562 & -806 & 4182 & 64 \\
\hline$H(33 D)$ & 7141 & -1090 & 4541 & 64 \\
\hline $\mathrm{H}(34 \mathrm{C})$ & 6726 & 320 & 4217 & 57 \\
\hline$H(34 D)$ & 6957 & 327 & 3671 & 57 \\
\hline $\mathrm{H}(35 \mathrm{C})$ & 5935 & -437 & 3727 & 41 \\
\hline$H(35 D)$ & 6076 & -109 & 3159 & 41 \\
\hline $\mathrm{H}(1 \mathrm{SA})$ & 4883 & 1057 & 3208 & 43 \\
\hline $\mathrm{H}(1 \mathrm{SB})$ & 4287 & 849 & 2682 & 43 \\
\hline $\mathrm{H}(2 \mathrm{SA})$ & 4388 & 2165 & 2170 & 34 \\
\hline $\mathrm{H}(2 \mathrm{SB})$ & 4692 & 2557 & 2828 & 34 \\
\hline $\mathrm{H}(5 \mathrm{SA})$ & 2284 & 2726 & 807 & 58 \\
\hline $\mathrm{H}(5 \mathrm{SB})$ & 2885 & 3220 & 848 & 58 \\
\hline $\mathrm{H}(6 \mathrm{SA})$ & 1835 & 4084 & 730 & 58 \\
\hline
\end{tabular}




\begin{tabular}{lrrrr}
$\mathrm{H}(6 \mathrm{SD})$ & 2482 & 4482 & 1053 & 58 \\
$\mathrm{H}(7 \mathrm{SA})$ & 2420 & 5224 & 336 & 58 \\
$\mathrm{H}(7 \mathrm{SD})$ & 1793 & 4764 & -2 & 58 \\
$\mathrm{H}(8 \mathrm{SA})$ & 2296 & 4118 & -505 & 58 \\
$\mathrm{H}(8 \mathrm{SB})$ & 2885 & 4101 & 45 & 58 \\
$\mathrm{H}(5 \mathrm{SC})$ & 2735 & 3437 & 1185 & 58 \\
$\mathrm{H}(5 \mathrm{SD})$ & 2364 & 2716 & 689 & 58 \\
$\mathrm{H}(6 \mathrm{SB})$ & 1675 & 3707 & 636 & 58 \\
$\mathrm{H}(6 \mathrm{SC})$ & 2113 & 4586 & 882 & 58 \\
$\mathrm{H}(7 \mathrm{SB})$ & 1906 & 5006 & 52 & 58 \\
$\mathrm{H}(7 \mathrm{SC})$ & 1593 & 4006 & -190 & 58 \\
$\mathrm{H}(8 \mathrm{SC})$ & 2333 & 3538 & -372 & 58 \\
$\mathrm{H}(8 \mathrm{SD})$ & 2628 & 4556 & -170 & 58 \\
& & & & \\
\hline
\end{tabular}


Table for Compound 2: $\left[\mathrm{Ca}_{2}(\mathrm{OMes})(\boldsymbol{\mu} \text {-OMes })_{2}(\mathrm{OH})(\mathrm{thf})_{3}\right]_{2} \cdot \mathbf{3 T H F}$ 
Table 1. Crystal data and structure refinement for compound 2.

Identification code

Empirical formula

Formula weight

Temperature

Wavelength

Crystal system

Space group

Unit cell dimensions

Volume

Z

Density (calculated)

Absorption coefficient

$\mathrm{F}(000)$

Crystal size

Theta range for data collection

Index ranges

Reflections collected

Independent reflections

Completeness to theta $=25.00^{\circ}$

Absorption correction

Max. and min. transmission

Refinement method

Data / restraints / parameters

Goodness-of-fit on $\mathrm{F}^{2}$

Final $\mathrm{R}$ indices [I $>2 \operatorname{sigma}(\mathrm{I})]$

$\mathrm{R}$ indices (all data)

Largest diff. peak and hole sadsq1

C78 H116 Ca4 O14

1438.03

96(2) K

$0.71073 \AA$

Triclinic

P-1

$\mathrm{a}=13.1767(15) \AA$

$\alpha=60.771(2)^{\circ}$.

$\mathrm{b}=14.5719(17) \AA$

$\beta=80.596(2)^{\circ}$.

$c=14.7956(17) \AA$

$\gamma=81.263(2)^{\circ}$.

2437.3(5) $\AA^{3}$

1

$0.980 \mathrm{Mg} / \mathrm{m}^{3}$

$0.270 \mathrm{~mm}^{-1}$

776

$0.48 \times 0.46 \times 0.42 \mathrm{~mm}^{3}$

1.61 to $25.00^{\circ}$.

$-15<=\mathrm{h}<=15,-17<=\mathrm{k}<=17,-17<=1<=17$

19982

$8591[\mathrm{R}(\mathrm{int})=0.0537]$

$99.9 \%$

Semi-empirical from equivalents

0.8950 and 0.8813

Full-matrix least-squares on $\mathrm{F}^{2}$

$8591 / 1 / 437$

0.964

$\mathrm{R} 1=0.0667, \mathrm{wR} 2=0.1790$

$\mathrm{R} 1=0.0967, \mathrm{wR} 2=0.1943$

0.687 and -0.549 e. $\AA^{-3}$ 


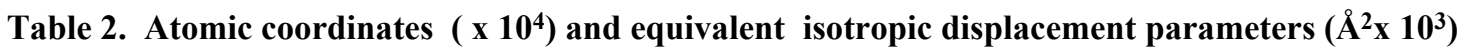
for compound 2. $U(e q)$ is defined as one third of the trace of the orthogonalized $U^{i j}$ tensor.

\begin{tabular}{|c|c|c|c|c|}
\hline & $\mathrm{x}$ & $\mathrm{y}$ & $\mathrm{z}$ & $\mathrm{U}(\mathrm{eq})$ \\
\hline $\mathrm{O}(1)$ & $328(2)$ & $8931(2)$ & $5655(2)$ & $22(1)$ \\
\hline $\mathrm{O}(2)$ & $1119(2)$ & $9361(2)$ & $3514(2)$ & $24(1)$ \\
\hline $\mathrm{C}(1)$ & $1310(3)$ & $9460(3)$ & $2545(3)$ & $24(1)$ \\
\hline$C(2)$ & 1911(3) & $8662(3)$ & $2386(3)$ & $26(1)$ \\
\hline$C(3)$ & 2037(3) & $8739(3)$ & 1397(3) & $35(1)$ \\
\hline$C(4)$ & 1611(3) & $9586(3)$ & $560(3)$ & $36(1)$ \\
\hline$C(5)$ & 1064(3) & 10391(3) & $717(3)$ & $36(1)$ \\
\hline$C(6)$ & $907(3)$ & $10357(3)$ & 1698(3) & $29(1)$ \\
\hline$C(7)$ & 2411(3) & 7733(3) & $3275(3)$ & $30(1)$ \\
\hline $\mathrm{C}(8)$ & $1759(4)$ & $9655(4)$ & $-509(3)$ & $51(1)$ \\
\hline$C(9)$ & $347(3)$ & $11267(3)$ & $1829(3)$ & $35(1)$ \\
\hline $\mathrm{Ca}(1)$ & $1350(1)$ & $10235(1)$ & $4400(1)$ & $21(1)$ \\
\hline $\mathrm{O}(3)$ & $1503(2)$ & $11368(2)$ & $5072(2)$ & $26(1)$ \\
\hline$C(10)$ & $2240(3)$ & $11606(3)$ & $5441(3)$ & $25(1)$ \\
\hline $\mathrm{C}(11)$ & 2693(3) & $10820(3)$ & $6332(3)$ & $28(1)$ \\
\hline$C(12)$ & $3481(3)$ & $11078(3)$ & $6689(3)$ & $32(1)$ \\
\hline$C(13)$ & $3808(3)$ & 12081(3) & $6214(3)$ & $33(1)$ \\
\hline$C(14)$ & $3313(3)$ & $12859(3)$ & $5358(3)$ & $32(1)$ \\
\hline$C(15)$ & $2524(3)$ & $12638(3)$ & 4977(3) & $26(1)$ \\
\hline$C(16)$ & $2299(3)$ & $9756(3)$ & $6940(3)$ & $35(1)$ \\
\hline$C(17)$ & 4649(3) & $12343(4)$ & $6623(4)$ & $48(1)$ \\
\hline$C(18)$ & 1962(3) & $13538(3)$ & $4106(3)$ & $35(1)$ \\
\hline $\mathrm{O}(4)$ & $2863(2)$ & 8992(2) & $5088(2)$ & $27(1)$ \\
\hline$C(19)$ & $3900(3)$ & 9261(3) & $5004(3)$ & $32(1)$ \\
\hline$C(20)$ & 4433(3) & $8340(3)$ & $5857(3)$ & $37(1)$ \\
\hline $\mathrm{C}(21)$ & $3898(3)$ & $7416(3)$ & 5985(3) & $34(1)$ \\
\hline$C(22)$ & $2800(3)$ & 7886(3) & $5830(3)$ & $29(1)$ \\
\hline $\mathrm{O}(5)$ & $2658(2)$ & $11143(2)$ & $2968(2)$ & $29(1)$ \\
\hline$C(23)$ & $3278(3)$ & 10711(3) & $2338(3)$ & $34(1)$ \\
\hline $\mathrm{C}(24)$ & $4276(3)$ & $11223(3)$ & $2030(3)$ & $39(1)$ \\
\hline$C(25)$ & $3920(3)$ & $12330(3)$ & $1854(3)$ & $38(1)$ \\
\hline
\end{tabular}




\begin{tabular}{lrrrl}
$\mathrm{C}(26)$ & $2997(3)$ & $12153(3)$ & $2679(3)$ & $33(1)$ \\
$\mathrm{Ca}(2)$ & $189(1)$ & $7994(1)$ & $4784(1)$ & $22(1)$ \\
$\mathrm{O}(6)$ & $886(2)$ & $6500(2)$ & $5905(2)$ & $30(1)$ \\
$\mathrm{C}(27)$ & $1233(3)$ & $5710(3)$ & $6759(3)$ & $27(1)$ \\
$\mathrm{C}(28)$ & $2127(3)$ & $5051(3)$ & $6694(3)$ & $31(1)$ \\
$\mathrm{C}(29)$ & $2446(3)$ & $4190(3)$ & $7611(3)$ & $41(1)$ \\
$\mathrm{C}(30)$ & $1955(3)$ & $3954(3)$ & $8589(3)$ & $42(1)$ \\
$\mathrm{C}(31)$ & $1094(3)$ & $4621(3)$ & $8636(3)$ & $37(1)$ \\
$\mathrm{C}(32)$ & $733(3)$ & $5483(3)$ & $7758(3)$ & $32(1)$ \\
$\mathrm{C}(33)$ & $2700(3)$ & $5299(3)$ & $5657(3)$ & $38(1)$ \\
$\mathrm{C}(34)$ & $2304(4)$ & $2997(4)$ & $9559(4)$ & $67(2)$ \\
$\mathrm{C}(35)$ & $-198(3)$ & $6161(3)$ & $7865(3)$ & $43(1)$ \\
$\mathrm{O}(7)$ & $-200(2)$ & $7341(2)$ & $3693(2)$ & $28(1)$ \\
$\mathrm{C}(36)$ & $-3(3)$ & $6267(3)$ & $3890(3)$ & $36(1)$ \\
$\mathrm{C}(37)$ & $433(3)$ & $6351(3)$ & $2852(3)$ & $39(1)$ \\
$\mathrm{C}(38)$ & $-185(3)$ & $7314(3)$ & $2091(3)$ & $39(1)$ \\
$\mathrm{C}(39)$ & $-637(3)$ & $7905(3)$ & $2702(3)$ & $31(1)$ \\
& & & & \\
\hline
\end{tabular}


Table 3. Bond lengths $[\AA ̊]$ and angles $\left[^{\circ}\right]$ for compound 2.

\begin{tabular}{|c|c|c|c|}
\hline $\mathrm{O}(1)-\mathrm{Ca}(1)$ & $2.321(3)$ & $C(15)-C(18)$ & $1.515(5)$ \\
\hline $\mathrm{O}(1)-\mathrm{Ca}(2)$ & $2.331(3)$ & $\mathrm{O}(4)-\mathrm{C}(22)$ & $1.447(4)$ \\
\hline $\mathrm{O}(1)-\mathrm{Ca}(1) \# 1$ & $2.348(3)$ & $\mathrm{O}(4)-\mathrm{C}(19)$ & $1.449(4)$ \\
\hline $\mathrm{O}(2)-\mathrm{C}(1)$ & $1.353(4)$ & $C(19)-C(20)$ & $1.496(5)$ \\
\hline $\mathrm{O}(2)-\mathrm{Ca}(1)$ & $2.305(2)$ & $\mathrm{C}(20)-\mathrm{C}(21)$ & $1.529(5)$ \\
\hline $\mathrm{O}(2)-\mathrm{Ca}(2)$ & $2.310(2)$ & $C(21)-C(22)$ & $1.506(5)$ \\
\hline $\mathrm{C}(1)-\mathrm{C}(2)$ & $1.400(5)$ & $\mathrm{O}(5)-\mathrm{C}(26)$ & $1.439(4)$ \\
\hline$C(1)-C(6)$ & $1.403(5)$ & $\mathrm{O}(5)-\mathrm{C}(23)$ & $1.450(4)$ \\
\hline$C(2)-C(3)$ & $1.396(5)$ & $\mathrm{C}(23)-\mathrm{C}(24)$ & $1.504(6)$ \\
\hline$C(2)-C(7)$ & $1.506(5)$ & $C(24)-C(25)$ & $1.514(5)$ \\
\hline$C(3)-C(4)$ & $1.377(5)$ & $C(25)-C(26)$ & $1.529(5)$ \\
\hline$C(4)-C(5)$ & $1.380(6)$ & $\mathrm{Ca}(2)-\mathrm{O}(6)$ & $2.167(2)$ \\
\hline$C(4)-C(8)$ & $1.517(5)$ & $\mathrm{Ca}(2)-\mathrm{O}(3) \# 1$ & $2.300(2)$ \\
\hline$C(5)-C(6)$ & $1.409(5)$ & $\mathrm{Ca}(2)-\mathrm{O}(7)$ & $2.386(2)$ \\
\hline$C(6)-C(9)$ & $1.500(5)$ & $\mathrm{O}(6)-\mathrm{C}(27)$ & $1.319(4)$ \\
\hline $\mathrm{Ca}(1)-\mathrm{O}(1) \# 1$ & $2.348(3)$ & $C(27)-C(32)$ & $1.413(5)$ \\
\hline $\mathrm{Ca}(1)-\mathrm{O}(3)$ & $2.351(2)$ & $\mathrm{C}(27)-\mathrm{C}(28)$ & $1.424(5)$ \\
\hline $\mathrm{Ca}(1)-\mathrm{O}(5)$ & $2.443(3)$ & $C(28)-C(29)$ & $1.398(5)$ \\
\hline $\mathrm{Ca}(1)-\mathrm{O}(4)$ & $2.461(2)$ & $C(28)-C(33)$ & $1.489(6)$ \\
\hline $\mathrm{O}(3)-\mathrm{C}(10)$ & $1.349(4)$ & $\mathrm{C}(29)-\mathrm{C}(30)$ & $1.381(6)$ \\
\hline $\mathrm{O}(3)-\mathrm{Ca}(2) \# 1$ & $2.300(2)$ & $C(30)-C(31)$ & $1.394(6)$ \\
\hline$C(10)-C(15)$ & $1.397(5)$ & $\mathrm{C}(30)-\mathrm{C}(34)$ & $1.510(6)$ \\
\hline$C(10)-C(11)$ & $1.407(5)$ & $C(31)-C(32)$ & $1.386(5)$ \\
\hline$C(11)-C(12)$ & $1.411(5)$ & $C(32)-C(35)$ & $1.492(6)$ \\
\hline$C(11)-C(16)$ & $1.488(5)$ & $\mathrm{O}(7)-\mathrm{C}(36)$ & $1.434(4)$ \\
\hline$C(12)-C(13)$ & $1.380(6)$ & $\mathrm{O}(7)-\mathrm{C}(39)$ & $1.454(4)$ \\
\hline$C(13)-C(14)$ & $1.400(5)$ & $C(36)-C(37)$ & $1.501(6)$ \\
\hline$C(13)-C(17)$ & $1.521(5)$ & $\mathrm{C}(37)-\mathrm{C}(38)$ & $1.520(5)$ \\
\hline$C(14)-C(15)$ & $1.405(5)$ & $\mathrm{C}(38)-\mathrm{C}(39)$ & $1.530(5)$ \\
\hline
\end{tabular}




\begin{tabular}{|c|c|c|c|}
\hline $\mathrm{Ca}(1)-\mathrm{O}(1)-\mathrm{Ca}(2)$ & $100.27(9)$ & $\mathrm{C}(10)-\mathrm{O}(3)-\mathrm{Ca}(1)$ & $137.8(2)$ \\
\hline $\mathrm{Ca}(1)-\mathrm{O}(1)-\mathrm{Ca}(1) \# 1$ & $105.22(10)$ & $\mathrm{Ca}(2) \# 1-\mathrm{O}(3)-\mathrm{Ca}(1)$ & $101.00(9)$ \\
\hline $\mathrm{Ca}(2)-\mathrm{O}(1)-\mathrm{Ca}(1) \# 1$ & $100.17(10)$ & $\mathrm{O}(3)-\mathrm{C}(10)-\mathrm{C}(15)$ & $120.6(3)$ \\
\hline $\mathrm{C}(1)-\mathrm{O}(2)-\mathrm{Ca}(1)$ & $139.8(2)$ & $\mathrm{O}(3)-\mathrm{C}(10)-\mathrm{C}(11)$ & $119.9(3)$ \\
\hline $\mathrm{C}(1)-\mathrm{O}(2)-\mathrm{Ca}(2)$ & $118.7(2)$ & $C(15)-C(10)-C(11)$ & $119.5(3)$ \\
\hline $\mathrm{Ca}(1)-\mathrm{O}(2)-\mathrm{Ca}(2)$ & $101.37(9)$ & $\mathrm{C}(10)-\mathrm{C}(11)-\mathrm{C}(12)$ & $119.0(4)$ \\
\hline $\mathrm{O}(2)-\mathrm{C}(1)-\mathrm{C}(2)$ & $119.9(3)$ & $\mathrm{C}(10)-\mathrm{C}(11)-\mathrm{C}(16)$ & $120.9(3)$ \\
\hline $\mathrm{O}(2)-\mathrm{C}(1)-\mathrm{C}(6)$ & $120.3(3)$ & $\mathrm{C}(12)-\mathrm{C}(11)-\mathrm{C}(16)$ & $119.9(3)$ \\
\hline $\mathrm{C}(2)-\mathrm{C}(1)-\mathrm{C}(6)$ & $119.8(3)$ & $\mathrm{C}(13)-\mathrm{C}(12)-\mathrm{C}(11)$ & $122.5(4)$ \\
\hline $\mathrm{C}(3)-\mathrm{C}(2)-\mathrm{C}(1)$ & 119.1(3) & $\mathrm{C}(12)-\mathrm{C}(13)-\mathrm{C}(14)$ & $117.4(3)$ \\
\hline $\mathrm{C}(3)-\mathrm{C}(2)-\mathrm{C}(7)$ & $120.7(3)$ & $\mathrm{C}(12)-\mathrm{C}(13)-\mathrm{C}(17)$ & $121.5(4)$ \\
\hline $\mathrm{C}(1)-\mathrm{C}(2)-\mathrm{C}(7)$ & $120.2(3)$ & $\mathrm{C}(14)-\mathrm{C}(13)-\mathrm{C}(17)$ & $121.0(4)$ \\
\hline$C(4)-C(3)-C(2)$ & $122.4(4)$ & $C(13)-C(14)-C(15)$ & $122.0(4)$ \\
\hline $\mathrm{C}(3)-\mathrm{C}(4)-\mathrm{C}(5)$ & $117.8(3)$ & $\mathrm{C}(10)-\mathrm{C}(15)-\mathrm{C}(14)$ & $119.5(3)$ \\
\hline$C(3)-C(4)-C(8)$ & $121.6(4)$ & $\mathrm{C}(10)-\mathrm{C}(15)-\mathrm{C}(18)$ & $121.5(3)$ \\
\hline $\mathrm{C}(5)-\mathrm{C}(4)-\mathrm{C}(8)$ & $120.6(4)$ & $\mathrm{C}(14)-\mathrm{C}(15)-\mathrm{C}(18)$ & $119.0(3)$ \\
\hline$C(4)-C(5)-C(6)$ & $122.5(4)$ & $\mathrm{C}(22)-\mathrm{O}(4)-\mathrm{C}(19)$ & $108.5(3)$ \\
\hline $\mathrm{C}(1)-\mathrm{C}(6)-\mathrm{C}(5)$ & $118.3(3)$ & $\mathrm{C}(22)-\mathrm{O}(4)-\mathrm{Ca}(1)$ & $123.8(2)$ \\
\hline $\mathrm{C}(1)-\mathrm{C}(6)-\mathrm{C}(9)$ & $120.8(3)$ & $\mathrm{C}(19)-\mathrm{O}(4)-\mathrm{Ca}(1)$ & $126.6(2)$ \\
\hline$C(5)-C(6)-C(9)$ & $121.0(3)$ & $\mathrm{O}(4)-\mathrm{C}(19)-\mathrm{C}(20)$ & $106.9(3)$ \\
\hline $\mathrm{O}(2)-\mathrm{Ca}(1)-\mathrm{O}(1)$ & $79.24(9)$ & $C(19)-C(20)-C(21)$ & $102.0(3)$ \\
\hline $\mathrm{O}(2)-\mathrm{Ca}(1)-\mathrm{O}(1) \# 1$ & $96.85(9)$ & $\mathrm{C}(22)-\mathrm{C}(21)-\mathrm{C}(20)$ & $101.9(3)$ \\
\hline $\mathrm{O}(1)-\mathrm{Ca}(1)-\mathrm{O}(1) \# 1$ & $74.78(10)$ & $\mathrm{O}(4)-\mathrm{C}(22)-\mathrm{C}(21)$ & $106.0(3)$ \\
\hline $\mathrm{O}(2)-\mathrm{Ca}(1)-\mathrm{O}(3)$ & $171.02(9)$ & $\mathrm{C}(26)-\mathrm{O}(5)-\mathrm{C}(23)$ & 109.2(3) \\
\hline $\mathrm{O}(1)-\mathrm{Ca}(1)-\mathrm{O}(3)$ & $106.40(9)$ & $\mathrm{C}(26)-\mathrm{O}(5)-\mathrm{Ca}(1)$ & $125.2(2)$ \\
\hline $\mathrm{O}(1) \# 1-\mathrm{Ca}(1)-\mathrm{O}(3)$ & $78.29(8)$ & $\mathrm{C}(23)-\mathrm{O}(5)-\mathrm{Ca}(1)$ & $125.5(2)$ \\
\hline $\mathrm{O}(2)-\mathrm{Ca}(1)-\mathrm{O}(5)$ & $86.10(9)$ & $\mathrm{O}(5)-\mathrm{C}(23)-\mathrm{C}(24)$ & $104.3(3)$ \\
\hline $\mathrm{O}(1)-\mathrm{Ca}(1)-\mathrm{O}(5)$ & $161.63(9)$ & $C(23)-C(24)-C(25)$ & $102.7(3)$ \\
\hline $\mathrm{O}(1) \# 1-\mathrm{Ca}(1)-\mathrm{O}(5)$ & $118.33(9)$ & $C(24)-C(25)-C(26)$ & $102.7(3)$ \\
\hline $\mathrm{O}(3)-\mathrm{Ca}(1)-\mathrm{O}(5)$ & $89.58(9)$ & $\mathrm{O}(5)-\mathrm{C}(26)-\mathrm{C}(25)$ & $106.7(3)$ \\
\hline $\mathrm{O}(2)-\mathrm{Ca}(1)-\mathrm{O}(4)$ & $88.86(8)$ & $\mathrm{O}(6)-\mathrm{Ca}(2)-\mathrm{O}(3) \# 1$ & $125.53(10)$ \\
\hline $\mathrm{O}(1)-\mathrm{Ca}(1)-\mathrm{O}(4)$ & $87.62(9)$ & $\mathrm{O}(6)-\mathrm{Ca}(2)-\mathrm{O}(2)$ & $123.94(10)$ \\
\hline $\mathrm{O}(1) \# 1-\mathrm{Ca}(1)-\mathrm{O}(4)$ & $160.04(9)$ & $\mathrm{O}(3) \# 1-\mathrm{Ca}(2)-\mathrm{O}(2)$ & 108.67(9) \\
\hline $\mathrm{O}(3)-\mathrm{Ca}(1)-\mathrm{O}(4)$ & $98.25(8)$ & $\mathrm{O}(6)-\mathrm{Ca}(2)-\mathrm{O}(1)$ & $96.34(9)$ \\
\hline $\mathrm{O}(5)-\mathrm{Ca}(1)-\mathrm{O}(4)$ & $81.04(8)$ & $\mathrm{O}(3) \# 1-\mathrm{Ca}(2)-\mathrm{O}(1)$ & $79.65(9)$ \\
\hline $\mathrm{C}(10)-\mathrm{O}(3)-\mathrm{Ca}(2) \# 1$ & $120.8(2)$ & $\mathrm{O}(2)-\mathrm{Ca}(2)-\mathrm{O}(1)$ & $78.93(8)$ \\
\hline
\end{tabular}




$\begin{array}{lrll}\mathrm{O}(6)-\mathrm{Ca}(2)-\mathrm{O}(7) & 95.27(9) & \mathrm{C}(29)-\mathrm{C}(30)-\mathrm{C}(34) & 121.9(4) \\ \mathrm{O}(3) \# 1-\mathrm{Ca}(2)-\mathrm{O}(7) & 91.00(8) & \mathrm{C}(31)-\mathrm{C}(30)-\mathrm{C}(34) & 121.4(4) \\ \mathrm{O}(2)-\mathrm{Ca}(2)-\mathrm{O}(7) & 97.16(8) & \mathrm{C}(32)-\mathrm{C}(31)-\mathrm{C}(30) & 122.8(4) \\ \mathrm{O}(1)-\mathrm{Ca}(2)-\mathrm{O}(7) & 167.96(9) & \mathrm{C}(31)-\mathrm{C}(32)-\mathrm{C}(27) & 120.1(4) \\ \mathrm{C}(27)-\mathrm{O}(6)-\mathrm{Ca}(2) & 164.0(2) & \mathrm{C}(31)-\mathrm{C}(32)-\mathrm{C}(35) & 119.9(4) \\ \mathrm{O}(6)-\mathrm{C}(27)-\mathrm{C}(32) & 121.6(3) & \mathrm{C}(27)-\mathrm{C}(32)-\mathrm{C}(35) & 120.0(3) \\ \mathrm{O}(6)-\mathrm{C}(27)-\mathrm{C}(28) & 120.3(3) & \mathrm{C}(36)-\mathrm{O}(7)-\mathrm{C}(39) & 105.2(3) \\ \mathrm{C}(32)-\mathrm{C}(27)-\mathrm{C}(28) & 118.1(3) & \mathrm{C}(36)-\mathrm{O}(7)-\mathrm{Ca}(2) & 125.2(2) \\ \mathrm{C}(29)-\mathrm{C}(28)-\mathrm{C}(27) & 118.9(4) & \mathrm{C}(39)-\mathrm{O}(7)-\mathrm{Ca}(2) & 129.5(2) \\ \mathrm{C}(29)-\mathrm{C}(28)-\mathrm{C}(33) & 121.8(4) & \mathrm{O}(7)-\mathrm{C}(36)-\mathrm{C}(37) & 104.3(3) \\ \mathrm{C}(27)-\mathrm{C}(28)-\mathrm{C}(33) & 119.4(3) & \mathrm{C}(36)-\mathrm{C}(37)-\mathrm{C}(38) & 103.2(3) \\ \mathrm{C}(30)-\mathrm{C}(29)-\mathrm{C}(28) & 123.5(4) & \mathrm{C}(37)-\mathrm{C}(38)-\mathrm{C}(39) & 104.4(3) \\ \mathrm{C}(29)-\mathrm{C}(30)-\mathrm{C}(31) & 116.7(4) & \mathrm{O}(7)-\mathrm{C}(39)-\mathrm{C}(38) & 106.2(3)\end{array}$

Symmetry transformations used to generate equivalent atoms:

$\# 1-\mathrm{x},-\mathrm{y}+2,-\mathrm{z}+1$ 
Table 4. Anisotropic displacement parameters $\left(\AA^{2} \times 10^{3}\right)$ for compound 2. The anisotropic displacement factor exponent takes the form: $-2 \pi^{2}\left[h^{2} a^{* 2} U^{11}+\ldots+2 h k^{*} b^{*} U^{12}\right]$

\begin{tabular}{|c|c|c|c|c|c|c|}
\hline & $\mathrm{U}^{11}$ & $\mathrm{U}^{22}$ & $\mathrm{U}^{33}$ & $\mathrm{U}^{23}$ & $\mathrm{U}^{13}$ & $\mathrm{U}^{12}$ \\
\hline $\mathrm{O}(1)$ & $27(1)$ & $24(1)$ & $18(1)$ & $-11(1)$ & $-6(1)$ & $1(1)$ \\
\hline $\mathrm{O}(2)$ & $30(1)$ & $30(1)$ & $20(1)$ & $-16(1)$ & $-7(1)$ & $1(1)$ \\
\hline$C(1)$ & $28(2)$ & $34(2)$ & $20(2)$ & $-18(2)$ & $-7(2)$ & $-5(2)$ \\
\hline$C(2)$ & $28(2)$ & $33(2)$ & $24(2)$ & $-18(2)$ & $-7(2)$ & $-2(2)$ \\
\hline$C(3)$ & $38(2)$ & $45(2)$ & $34(2)$ & $-30(2)$ & $-5(2)$ & $2(2)$ \\
\hline $\mathrm{C}(4)$ & $41(2)$ & $52(3)$ & $21(2)$ & $-21(2)$ & $-6(2)$ & $-4(2)$ \\
\hline$C(5)$ & $41(2)$ & $44(2)$ & $21(2)$ & $-12(2)$ & $-11(2)$ & $1(2)$ \\
\hline$C(6)$ & $28(2)$ & $39(2)$ & $27(2)$ & $-20(2)$ & $-6(2)$ & $-1(2)$ \\
\hline$C(7)$ & $33(2)$ & $36(2)$ & $33(2)$ & $-25(2)$ & $-8(2)$ & $5(2)$ \\
\hline $\mathrm{C}(8)$ & $55(3)$ & $78(3)$ & $30(2)$ & $-34(2)$ & $-9(2)$ & $3(3)$ \\
\hline $\mathrm{C}(9)$ & $35(2)$ & $36(2)$ & $30(2)$ & $-15(2)$ & $-11(2)$ & $7(2)$ \\
\hline $\mathrm{Ca}(1)$ & $22(1)$ & $25(1)$ & $23(1)$ & $-16(1)$ & $-5(1)$ & 1(1) \\
\hline $\mathrm{O}(3)$ & $25(1)$ & $33(1)$ & $32(1)$ & $-24(1)$ & $-8(1)$ & $0(1)$ \\
\hline$C(10)$ & $22(2)$ & $35(2)$ & $32(2)$ & $-27(2)$ & $-5(2)$ & $2(2)$ \\
\hline $\mathrm{C}(11)$ & $29(2)$ & $34(2)$ & $32(2)$ & $-25(2)$ & $-8(2)$ & $8(2)$ \\
\hline$C(12)$ & $29(2)$ & $45(2)$ & $36(2)$ & $-30(2)$ & $-14(2)$ & $13(2)$ \\
\hline$C(13)$ & $24(2)$ & $50(3)$ & $46(2)$ & $-39(2)$ & $-7(2)$ & $4(2)$ \\
\hline$C(14)$ & $30(2)$ & $37(2)$ & $41(2)$ & $-29(2)$ & $-4(2)$ & $-4(2)$ \\
\hline$C(15)$ & $24(2)$ & $31(2)$ & $30(2)$ & $-21(2)$ & $-4(2)$ & $3(2)$ \\
\hline$C(16)$ & $47(3)$ & $34(2)$ & $31(2)$ & $-21(2)$ & $-11(2)$ & $2(2)$ \\
\hline$C(17)$ & $34(2)$ & $72(3)$ & $66(3)$ & $-53(3)$ & $-19(2)$ & $4(2)$ \\
\hline$C(18)$ & $40(2)$ & $32(2)$ & $39(2)$ & $-21(2)$ & $-8(2)$ & $-2(2)$ \\
\hline $\mathrm{O}(4)$ & $27(1)$ & $32(1)$ & $27(1)$ & $-17(1)$ & $-11(1)$ & $0(1)$ \\
\hline$C(19)$ & $31(2)$ & $32(2)$ & $37(2)$ & $-20(2)$ & $-4(2)$ & $-3(2)$ \\
\hline$C(20)$ & $31(2)$ & $46(2)$ & $43(2)$ & $-26(2)$ & $-15(2)$ & $4(2)$ \\
\hline $\mathrm{C}(21)$ & $33(2)$ & $31(2)$ & $36(2)$ & $-15(2)$ & $-8(2)$ & $3(2)$ \\
\hline$C(22)$ & $35(2)$ & $27(2)$ & $32(2)$ & $-19(2)$ & $-7(2)$ & $0(2)$ \\
\hline $\mathrm{O}(5)$ & $29(1)$ & $34(1)$ & $30(1)$ & $-19(1)$ & $-1(1)$ & $-3(1)$ \\
\hline $\mathrm{C}(23)$ & $34(2)$ & $43(2)$ & $33(2)$ & $-26(2)$ & $-1(2)$ & $0(2)$ \\
\hline$C(24)$ & $36(2)$ & $48(3)$ & $41(2)$ & $-29(2)$ & $1(2)$ & $-2(2)$ \\
\hline $\mathrm{C}(25)$ & $44(3)$ & $39(2)$ & $32(2)$ & $-16(2)$ & $-3(2)$ & $-9(2)$ \\
\hline
\end{tabular}




\begin{tabular}{lllllll}
$\mathrm{C}(26)$ & $30(2)$ & $37(2)$ & $36(2)$ & $-22(2)$ & $-5(2)$ & $1(2)$ \\
$\mathrm{Ca}(2)$ & $25(1)$ & $24(1)$ & $23(1)$ & $-16(1)$ & $-9(1)$ & $3(1)$ \\
$\mathrm{O}(6)$ & $37(2)$ & $25(1)$ & $29(1)$ & $-14(1)$ & $-14(1)$ & $6(1)$ \\
$\mathrm{C}(27)$ & $33(2)$ & $23(2)$ & $32(2)$ & $-15(2)$ & $-16(2)$ & $-1(2)$ \\
$\mathrm{C}(28)$ & $35(2)$ & $29(2)$ & $35(2)$ & $-17(2)$ & $-14(2)$ & $-2(2)$ \\
$\mathrm{C}(29)$ & $42(3)$ & $26(2)$ & $50(3)$ & $-12(2)$ & $-21(2)$ & $5(2)$ \\
$\mathrm{C}(30)$ & $48(3)$ & $37(2)$ & $38(2)$ & $-7(2)$ & $-22(2)$ & $-9(2)$ \\
$\mathrm{C}(31)$ & $46(3)$ & $41(2)$ & $29(2)$ & $-16(2)$ & $-12(2)$ & $-12(2)$ \\
$\mathrm{C}(32)$ & $44(2)$ & $27(2)$ & $31(2)$ & $-16(2)$ & $-13(2)$ & $-6(2)$ \\
$\mathrm{C}(33)$ & $40(2)$ & $35(2)$ & $46(2)$ & $-27(2)$ & $-13(2)$ & $11(2)$ \\
$\mathrm{C}(34)$ & $56(3)$ & $58(3)$ & $60(3)$ & $0(3)$ & $-32(3)$ & $-5(3)$ \\
$\mathrm{C}(35)$ & $53(3)$ & $47(3)$ & $30(2)$ & $-19(2)$ & $-5(2)$ & $-1(2)$ \\
$\mathrm{O}(7)$ & $36(2)$ & $27(1)$ & $29(1)$ & $-19(1)$ & $-13(1)$ & $3(1)$ \\
$\mathrm{C}(36)$ & $47(3)$ & $27(2)$ & $44(2)$ & $-21(2)$ & $-19(2)$ & $-4(2)$ \\
$\mathrm{C}(37)$ & $47(3)$ & $36(2)$ & $53(3)$ & $-34(2)$ & $-12(2)$ & $-2(2)$ \\
$\mathrm{C}(38)$ & $44(3)$ & $51(3)$ & $40(2)$ & $-35(2)$ & $-11(2)$ & $-2(2)$ \\
$\mathrm{C}(39)$ & $36(2)$ & $34(2)$ & $33(2)$ & $-21(2)$ & $-14(2)$ & $1(2)$ \\
& & & & & & \\
\hline
\end{tabular}


Table 5. Hydrogen coordinates $\left(\times 10^{4}\right)$ and isotropic displacement parameters $\left(\AA^{2} \times 10^{3}\right)$ for compound 2.

\begin{tabular}{|c|c|c|c|c|}
\hline & $\mathrm{x}$ & $\mathrm{y}$ & $\mathrm{z}$ & $\mathrm{U}(\mathrm{eq})$ \\
\hline $\mathrm{H}(1 \mathrm{~A})$ & $400(30)$ & $8560(30)$ & $6130(30)$ & 26 \\
\hline $\mathrm{H}(3 \mathrm{~A})$ & 2430 & 8186 & 1298 & 42 \\
\hline $\mathrm{H}(5 \mathrm{~A})$ & 783 & 10990 & 142 & 44 \\
\hline $\mathrm{H}(7 \mathrm{~A})$ & 2243 & 7813 & 3905 & 45 \\
\hline $\mathrm{H}(7 \mathrm{~B})$ & 3161 & 7702 & 3101 & 45 \\
\hline $\mathrm{H}(7 \mathrm{C})$ & 2153 & 7081 & 3398 & 45 \\
\hline $\mathrm{H}(8 \mathrm{~A})$ & 2163 & 9018 & -476 & 77 \\
\hline $\mathrm{H}(8 \mathrm{~B})$ & 2126 & 10277 & -1005 & 77 \\
\hline $\mathrm{H}(8 \mathrm{C})$ & 1084 & 9715 & -738 & 77 \\
\hline $\mathrm{H}(9 \mathrm{~A})$ & 314 & 11105 & 2558 & 52 \\
\hline $\mathrm{H}(9 \mathrm{~B})$ & -355 & 11391 & 1630 & 52 \\
\hline $\mathrm{H}(9 \mathrm{C})$ & 716 & 11901 & 1385 & 52 \\
\hline $\mathrm{H}(12 \mathrm{~A})$ & 3798 & 10540 & 7280 & 39 \\
\hline $\mathrm{H}(14 \mathrm{~A})$ & 3517 & 13558 & 5024 & 38 \\
\hline $\mathrm{H}(16 \mathrm{~A})$ & 2706 & 9317 & 7529 & 52 \\
\hline $\mathrm{H}(16 \mathrm{~B})$ & 2358 & 9426 & 6491 & 52 \\
\hline $\mathrm{H}(16 \mathrm{C})$ & 1573 & 9823 & 7200 & 52 \\
\hline $\mathrm{H}(17 \mathrm{~A})$ & 4773 & 13090 & 6185 & 72 \\
\hline $\mathrm{H}(17 \mathrm{~B})$ & 5286 & 11904 & 6608 & 72 \\
\hline $\mathrm{H}(17 \mathrm{C})$ & 4429 & 12206 & 7341 & 72 \\
\hline $\mathrm{H}(18 \mathrm{~A})$ & 1438 & 13261 & 3926 & 52 \\
\hline $\mathrm{H}(18 \mathrm{~B})$ & 2457 & 13886 & 3494 & 52 \\
\hline $\mathrm{H}(18 \mathrm{C})$ & 1628 & 14048 & 4335 & 52 \\
\hline $\mathrm{H}(19 \mathrm{~A})$ & 3880 & 9902 & 5081 & 38 \\
\hline $\mathrm{H}(19 \mathrm{~B})$ & 4270 & 9399 & 4316 & 38 \\
\hline $\mathrm{H}(20 \mathrm{~A})$ & 4327 & 8399 & 6505 & 45 \\
\hline $\mathrm{H}(20 \mathrm{~B})$ & 5183 & 8270 & 5651 & 45 \\
\hline $\mathrm{H}(21 \mathrm{~A})$ & 4193 & 7197 & 5453 & 40 \\
\hline $\mathrm{H}(21 \mathrm{~B})$ & 3949 & 6803 & 6687 & 40 \\
\hline $\mathrm{H}(22 \mathrm{~A})$ & 2444 & 7537 & 5556 & 35 \\
\hline
\end{tabular}




\begin{tabular}{|c|c|c|c|c|}
\hline $\mathrm{H}(22 \mathrm{~B})$ & 2417 & 7801 & 6497 & 35 \\
\hline $\mathrm{H}(23 \mathrm{~A})$ & 2938 & 10889 & 1716 & 40 \\
\hline $\mathrm{H}(23 \mathrm{~B})$ & 3398 & 9934 & 2745 & 40 \\
\hline $\mathrm{H}(24 \mathrm{~A})$ & 4640 & 11230 & 1386 & 47 \\
\hline $\mathrm{H}(24 \mathrm{~B})$ & 4737 & 10856 & 2593 & 47 \\
\hline $\mathrm{H}(25 \mathrm{~A})$ & 3710 & 12787 & 1143 & 46 \\
\hline $\mathrm{H}(25 \mathrm{~B})$ & 4468 & 12652 & 1961 & 46 \\
\hline $\mathrm{H}(26 \mathrm{~A})$ & 3203 & 12154 & 3293 & 39 \\
\hline $\mathrm{H}(26 \mathrm{~B})$ & 2436 & 12717 & 2394 & 39 \\
\hline $\mathrm{H}(29 \mathrm{~A})$ & 3032 & 3743 & 7558 & 49 \\
\hline $\mathrm{H}(31 \mathrm{~A})$ & 739 & 4478 & 9299 & 44 \\
\hline $\mathrm{H}(33 \mathrm{~A})$ & 3292 & 4777 & 5744 & 56 \\
\hline $\mathrm{H}(33 \mathrm{~B})$ & 2945 & 6005 & 5336 & 56 \\
\hline $\mathrm{H}(33 \mathrm{C})$ & 2241 & 5279 & 5210 & 56 \\
\hline $\mathrm{H}(34 \mathrm{~A})$ & 1863 & 2972 & 10170 & 100 \\
\hline $\mathrm{H}(34 \mathrm{~B})$ & 3022 & 3042 & 9622 & 100 \\
\hline $\mathrm{H}(34 \mathrm{C})$ & 2254 & 2358 & 9513 & 100 \\
\hline $\mathrm{H}(35 \mathrm{~A})$ & -448 & 5895 & 8604 & 64 \\
\hline $\mathrm{H}(35 \mathrm{~B})$ & -740 & 6144 & 7493 & 64 \\
\hline $\mathrm{H}(35 \mathrm{C})$ & -17 & 6888 & 7569 & 64 \\
\hline $\mathrm{H}(36 \mathrm{~A})$ & -648 & 5905 & 4147 & 43 \\
\hline $\mathrm{H}(36 \mathrm{~B})$ & 500 & 5874 & 4411 & 43 \\
\hline $\mathrm{H}(37 \mathrm{~A})$ & 331 & 5712 & 2814 & 47 \\
\hline $\mathrm{H}(37 \mathrm{~B})$ & 1178 & 6456 & 2714 & 47 \\
\hline $\mathrm{H}(38 \mathrm{~A})$ & -740 & 7103 & 1870 & 47 \\
\hline $\mathrm{H}(38 \mathrm{~B})$ & 267 & 7758 & 1466 & 47 \\
\hline $\mathrm{H}(39 \mathrm{~A})$ & -449 & 8643 & 2320 & 38 \\
\hline $\mathrm{H}(39 \mathrm{~B})$ & -1399 & 7911 & 2813 & 38 \\
\hline
\end{tabular}


Table for Compound 3: $\left[\mathrm{Ca}_{2}(\mathrm{OMes})(\boldsymbol{\mu} \text {-OMes })_{2}(\mathrm{OH})(\mathbf{e n})_{2}\right]_{2} \cdot \mathbf{2 P h M e}$ 
Table 1. Crystal data and structure refinement for compound 3.

Identification code

Empirical formula

Formula weight

Temperature

Wavelength

Crystal system

Space group

Unit cell dimensions

Volume

Z

Density (calculated)

Absorption coefficient

$\mathrm{F}(000)$

Crystal size

Theta range for data collection

Index ranges

Reflections collected

Independent reflections

Completeness to theta $=25.00^{\circ}$

Absorption correction

Max. and min. transmission

Refinement method

Data / restraints / parameters

Goodness-of-fit on $\mathrm{F}^{2}$

Final $\mathrm{R}$ indices [I $>2 \operatorname{sigma}(\mathrm{I})]$

$\mathrm{R}$ indices (all data)

Largest diff. peak and hole sadsq1

C76 H116 Ca4 N8 O8

1430.09

91(2) K

$0.71073 \AA$

Triclinic

P-1

$\mathrm{a}=12.3900(9) \AA$

$\alpha=116.3660(10)^{\circ}$.

$\mathrm{b}=13.8979(10) \AA$

$\beta=91.232(2)^{\circ}$.

$\mathrm{c}=14.9393(11) \AA$

$\gamma=110.3100(10)^{\circ}$.

2113.8(3) $\AA^{3}$

1

$1.123 \mathrm{Mg} / \mathrm{m}^{3}$

$0.309 \mathrm{~mm}^{-1}$

772

$0.40 \times 0.12 \times 0.06 \mathrm{~mm}^{3}$

1.56 to $25.00^{\circ}$.

$-14<=\mathrm{h}<=14,-16<=\mathrm{k}<=16,-17<=\mathrm{k}<=17$

17067

$7437[\mathrm{R}($ int $)=0.0414]$

$99.9 \%$

Semi-empirical from equivalents

0.9817 and 0.8865

Full-matrix least-squares on $\mathrm{F}^{2}$

7437 / 0 / 433

1.063

$\mathrm{R} 1=0.0599, \mathrm{wR} 2=0.1635$

$\mathrm{R} 1=0.0825, \mathrm{wR} 2=0.1736$

0.561 and $-0.580 \mathrm{e} . \AA^{-3}$ 


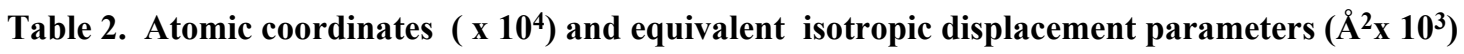
for compound 3. $U(e q)$ is defined as one third of the trace of the orthogonalized $U^{i j}$ tensor.

\begin{tabular}{|c|c|c|c|c|}
\hline & $\mathrm{x}$ & $\mathrm{y}$ & $\mathrm{z}$ & $\mathrm{U}(\mathrm{eq})$ \\
\hline $\mathrm{Ca}(1)$ & $8852(1)$ & $8565(1)$ & $4103(1)$ & $18(1)$ \\
\hline $\mathrm{Ca}(2)$ & 11231(1) & $10015(1)$ & $3173(1)$ & $18(1)$ \\
\hline $\mathrm{O}(1)$ & $9099(2)$ & $10398(2)$ & $5424(2)$ & $19(1)$ \\
\hline $\mathrm{O}(2)$ & $9265(2)$ & $8747(2)$ & $2653(2)$ & $24(1)$ \\
\hline$C(1)$ & $8597(3)$ & $8337(3)$ & $1746(3)$ & $23(1)$ \\
\hline$C(2)$ & 7957(3) & $8946(3)$ & $1595(3)$ & $26(1)$ \\
\hline$C(3)$ & $7320(3)$ & $8521(3)$ & $628(3)$ & $29(1)$ \\
\hline$C(4)$ & $7243(3)$ & 7503(3) & $-204(3)$ & $33(1)$ \\
\hline$C(5)$ & $7858(3)$ & 6902(3) & $-55(3)$ & $31(1)$ \\
\hline$C(6)$ & $8513(3)$ & $7280(3)$ & $894(3)$ & $24(1)$ \\
\hline$C(7)$ & 7984(3) & $10046(3)$ & 2487(3) & $38(1)$ \\
\hline $\mathrm{C}(8)$ & $6507(4)$ & 7034(4) & $-1246(3)$ & $49(1)$ \\
\hline $\mathrm{C}(9)$ & $9134(4)$ & $6576(3)$ & 1021(3) & $35(1)$ \\
\hline $\mathrm{O}(3)$ & $8405(2)$ & $8147(2)$ & $5431(2)$ & $21(1)$ \\
\hline$C(10)$ & $8070(3)$ & $7150(3)$ & $5480(2)$ & $20(1)$ \\
\hline$C(11)$ & $8816(3)$ & $6545(3)$ & $5331(2)$ & $22(1)$ \\
\hline$C(12)$ & $8458(3)$ & $5541(3)$ & $5436(3)$ & $27(1)$ \\
\hline$C(13)$ & $7373(3)$ & $5082(3)$ & $5664(3)$ & $29(1)$ \\
\hline$C(14)$ & $6642(3)$ & $5665(3)$ & $5782(3)$ & $28(1)$ \\
\hline$C(15)$ & $6957(3)$ & $6688(3)$ & $5703(3)$ & $22(1)$ \\
\hline$C(16)$ & 10001(3) & 6997(3) & $5086(3)$ & $28(1)$ \\
\hline $\mathrm{C}(17)$ & $7016(4)$ & $3985(4)$ & $5774(4)$ & $51(1)$ \\
\hline$C(18)$ & $6164(3)$ & $7340(3)$ & $5901(3)$ & $30(1)$ \\
\hline $\mathrm{O}(4)$ & $12727(2)$ & $9469(2)$ & $3126(2)$ & $28(1)$ \\
\hline$C(19)$ & 13094(3) & $8653(3)$ & $2522(3)$ & $24(1)$ \\
\hline$C(20)$ & $14020(3)$ & 8929(3) & 2024(3) & $28(1)$ \\
\hline $\mathrm{C}(21)$ & $14422(3)$ & $8070(3)$ & $1425(3)$ & $30(1)$ \\
\hline $\mathrm{C}(22)$ & 13944(3) & 6941(3) & $1282(3)$ & $27(1)$ \\
\hline$C(23)$ & $13029(3)$ & $6687(3)$ & 1775(3) & $26(1)$ \\
\hline$C(24)$ & 12591(3) & $7506(3)$ & $2382(3)$ & $24(1)$ \\
\hline$C(25)$ & $14594(4)$ & $10161(4)$ & 2181(4) & $46(1)$ \\
\hline
\end{tabular}




\begin{tabular}{lrrrr}
$\mathrm{C}(26)$ & $14386(3)$ & $6040(3)$ & $615(3)$ & $35(1)$ \\
$\mathrm{C}(27)$ & $11622(3)$ & $7197(3)$ & $2918(3)$ & $30(1)$ \\
$\mathrm{N}(1)$ & $7953(3)$ & $6257(2)$ & $3068(2)$ & $27(1)$ \\
$\mathrm{N}(2)$ & $6718(2)$ & $7780(3)$ & $3273(2)$ & $30(1)$ \\
$\mathrm{C}(28)$ & $6804(3)$ & $5846(3)$ & $2426(3)$ & $31(1)$ \\
$\mathrm{C}(29)$ & $6110(3)$ & $6541(3)$ & $3004(3)$ & $32(1)$ \\
$\mathrm{N}(3)$ & $11278(3)$ & $9083(3)$ & $1217(2)$ & $32(1)$ \\
$\mathrm{N}(4)$ & $11010(3)$ & $11247(3)$ & $2296(2)$ & $35(1)$ \\
$\mathrm{C}(30)$ & $11548(3)$ & $9937(3)$ & $841(3)$ & $35(1)$ \\
$\mathrm{C}(31)$ & $10780(3)$ & $10618(3)$ & $1183(3)$ & $32(1)$ \\
$\mathrm{C}(32)$ & $2467(4)$ & $6716(3)$ & $7788(3)$ & $42(1)$ \\
$\mathrm{C}(33)$ & $1324(4)$ & $6436(4)$ & $7349(4)$ & $53(1)$ \\
$\mathrm{C}(34)$ & $426(4)$ & $6246(5)$ & $7836(4)$ & $61(1)$ \\
$\mathrm{C}(35)$ & $636(5)$ & $6337(5)$ & $8788(4)$ & $61(1)$ \\
$\mathrm{C}(36)$ & $1746(4)$ & $6626(4)$ & $9241(3)$ & $49(1)$ \\
$\mathrm{C}(37)$ & $2660(4)$ & $6820(4)$ & $8743(3)$ & $45(1)$ \\
$\mathrm{C}(38)$ & $3456(5)$ & $6866(5)$ & $7220(4)$ & $67(2)$ \\
& & & & \\
\hline
\end{tabular}


Table 3. Bond lengths $[\AA ̊]$ and angles $\left[{ }^{\circ}\right]$ for compound 3 .

\begin{tabular}{|c|c|c|c|}
\hline $\mathrm{Ca}(1)-\mathrm{O}(3)$ & $2.329(2)$ & $C(11)-C(16)$ & $1.508(5)$ \\
\hline $\mathrm{Ca}(1)-\mathrm{O}(1)$ & $2.334(2)$ & $\mathrm{C}(12)-\mathrm{C}(13)$ & $1.396(5)$ \\
\hline $\mathrm{Ca}(1)-\mathrm{O}(2)$ & $2.338(2)$ & $C(13)-C(14)$ & $1.378(5)$ \\
\hline $\mathrm{Ca}(1)-\mathrm{O}(1) \# 1$ & $2.337(2)$ & $\mathrm{C}(13)-\mathrm{C}(17)$ & $1.518(5)$ \\
\hline $\mathrm{Ca}(1)-\mathrm{N}(2)$ & $2.525(3)$ & $\mathrm{C}(14)-\mathrm{C}(15)$ & $1.398(5)$ \\
\hline $\mathrm{Ca}(1)-\mathrm{N}(1)$ & $2.638(3)$ & $C(15)-C(18)$ & $1.505(5)$ \\
\hline $\mathrm{Ca}(2)-\mathrm{O}(4)$ & $2.226(2)$ & $\mathrm{O}(4)-\mathrm{C}(19)$ & $1.332(4)$ \\
\hline $\mathrm{Ca}(2)-\mathrm{O}(2)$ & $2.316(2)$ & $C(19)-C(24)$ & $1.409(5)$ \\
\hline $\mathrm{Ca}(2)-\mathrm{O}(3) \# 1$ & $2.346(2)$ & $\mathrm{C}(19)-\mathrm{C}(20)$ & $1.415(5)$ \\
\hline $\mathrm{Ca}(2)-\mathrm{O}(1) \# 1$ & $2.410(2)$ & $C(20)-C(21)$ & $1.399(5)$ \\
\hline $\mathrm{Ca}(2)-\mathrm{N}(3)$ & $2.629(3)$ & $\mathrm{C}(20)-\mathrm{C}(25)$ & $1.511(5)$ \\
\hline $\mathrm{Ca}(2)-\mathrm{N}(4)$ & $2.650(3)$ & $\mathrm{C}(21)-\mathrm{C}(22)$ & $1.381(5)$ \\
\hline $\mathrm{O}(1)-\mathrm{Ca}(1) \# 1$ & $2.337(2)$ & $\mathrm{C}(22)-\mathrm{C}(23)$ & $1.394(5)$ \\
\hline $\mathrm{O}(1)-\mathrm{Ca}(2) \# 1$ & $2.410(2)$ & $\mathrm{C}(22)-\mathrm{C}(26)$ & $1.501(5)$ \\
\hline $\mathrm{O}(2)-\mathrm{C}(1)$ & $1.336(4)$ & $\mathrm{C}(23)-\mathrm{C}(24)$ & $1.394(5)$ \\
\hline$C(1)-C(6)$ & $1.419(5)$ & $\mathrm{C}(24)-\mathrm{C}(27)$ & $1.502(5)$ \\
\hline$C(1)-C(2)$ & $1.422(5)$ & $\mathrm{N}(1)-\mathrm{C}(28)$ & $1.470(5)$ \\
\hline$C(2)-C(3)$ & $1.389(5)$ & $\mathrm{N}(2)-\mathrm{C}(29)$ & $1.472(5)$ \\
\hline$C(2)-C(7)$ & $1.506(5)$ & $\mathrm{C}(28)-\mathrm{C}(29)$ & $1.512(5)$ \\
\hline$C(3)-C(4)$ & $1.376(6)$ & $\mathrm{N}(3)-\mathrm{C}(30)$ & $1.469(5)$ \\
\hline$C(4)-C(5)$ & $1.390(6)$ & $\mathrm{N}(4)-\mathrm{C}(31)$ & $1.458(5)$ \\
\hline$C(4)-C(8)$ & $1.518(5)$ & $\mathrm{C}(30)-\mathrm{C}(31)$ & $1.510(5)$ \\
\hline$C(5)-C(6)$ & $1.391(5)$ & $\mathrm{C}(32)-\mathrm{C}(37)$ & $1.375(6)$ \\
\hline$C(6)-C(9)$ & $1.506(5)$ & $\mathrm{C}(32)-\mathrm{C}(33)$ & $1.393(6)$ \\
\hline $\mathrm{O}(3)-\mathrm{C}(10)$ & $1.339(4)$ & $\mathrm{C}(32)-\mathrm{C}(38)$ & $1.513(6)$ \\
\hline $\mathrm{O}(3)-\mathrm{Ca}(2) \# 1$ & $2.346(2)$ & $\mathrm{C}(33)-\mathrm{C}(34)$ & $1.356(7)$ \\
\hline$C(10)-C(11)$ & $1.411(5)$ & $\mathrm{C}(34)-\mathrm{C}(35)$ & $1.381(7)$ \\
\hline$C(10)-C(15)$ & $1.421(5)$ & $C(35)-C(36)$ & $1.359(7)$ \\
\hline$C(11)-C(12)$ & $1.392(5)$ & $\mathrm{C}(36)-\mathrm{C}(37)$ & $1.382(6)$ \\
\hline
\end{tabular}




\begin{tabular}{|c|c|c|c|}
\hline $\mathrm{O}(3)-\mathrm{Ca}(1)-\mathrm{O}(1)$ & $78.42(8)$ & $\mathrm{C}(3)-\mathrm{C}(2)-\mathrm{C}(1)$ & $119.7(3)$ \\
\hline $\mathrm{O}(3)-\mathrm{Ca}(1)-\mathrm{O}(2)$ & $173.07(8)$ & $C(3)-C(2)-C(7)$ & $120.9(3)$ \\
\hline $\mathrm{O}(1)-\mathrm{Ca}(1)-\mathrm{O}(2)$ & $108.50(8)$ & $\mathrm{C}(1)-\mathrm{C}(2)-\mathrm{C}(7)$ & $119.5(3)$ \\
\hline $\mathrm{O}(3)-\mathrm{Ca}(1)-\mathrm{O}(1) \# 1$ & $103.27(8)$ & $\mathrm{C}(4)-\mathrm{C}(3)-\mathrm{C}(2)$ & $123.0(4)$ \\
\hline $\mathrm{O}(1)-\mathrm{Ca}(1)-\mathrm{O}(1) \# 1$ & $76.45(8)$ & $\mathrm{C}(3)-\mathrm{C}(4)-\mathrm{C}(5)$ & $117.3(3)$ \\
\hline $\mathrm{O}(2)-\mathrm{Ca}(1)-\mathrm{O}(1) \# 1$ & $78.55(8)$ & $\mathrm{C}(3)-\mathrm{C}(4)-\mathrm{C}(8)$ & $122.1(4)$ \\
\hline $\mathrm{O}(3)-\mathrm{Ca}(1)-\mathrm{N}(2)$ & $93.56(9)$ & $\mathrm{C}(5)-\mathrm{C}(4)-\mathrm{C}(8)$ & $120.6(4)$ \\
\hline $\mathrm{O}(1)-\mathrm{Ca}(1)-\mathrm{N}(2)$ & $100.78(9)$ & $\mathrm{C}(4)-\mathrm{C}(5)-\mathrm{C}(6)$ & $122.5(3)$ \\
\hline $\mathrm{O}(2)-\mathrm{Ca}(1)-\mathrm{N}(2)$ & $85.49(9)$ & $C(5)-C(6)-C(1)$ & $119.8(3)$ \\
\hline $\mathrm{O}(1) \# 1-\mathrm{Ca}(1)-\mathrm{N}(2)$ & $161.89(9)$ & $\mathrm{C}(5)-\mathrm{C}(6)-\mathrm{C}(9)$ & $120.4(3)$ \\
\hline $\mathrm{O}(3)-\mathrm{Ca}(1)-\mathrm{N}(1)$ & $80.39(8)$ & $\mathrm{C}(1)-\mathrm{C}(6)-\mathrm{C}(9)$ & $119.8(3)$ \\
\hline $\mathrm{O}(1)-\mathrm{Ca}(1)-\mathrm{N}(1)$ & $155.92(9)$ & $\mathrm{C}(10)-\mathrm{O}(3)-\mathrm{Ca}(1)$ & 132.11(19) \\
\hline $\mathrm{O}(2)-\mathrm{Ca}(1)-\mathrm{N}(1)$ & $92.87(9)$ & $\mathrm{C}(10)-\mathrm{O}(3)-\mathrm{Ca}(2) \# 1$ & $124.25(18)$ \\
\hline $\mathrm{O}(1) \# 1-\mathrm{Ca}(1)-\mathrm{N}(1)$ & $119.69(9)$ & $\mathrm{Ca}(1)-\mathrm{O}(3)-\mathrm{Ca}(2) \# 1$ & $103.58(8)$ \\
\hline $\mathrm{N}(2)-\mathrm{Ca}(1)-\mathrm{N}(1)$ & $69.19(9)$ & $\mathrm{O}(3)-\mathrm{C}(10)-\mathrm{C}(11)$ & $121.1(3)$ \\
\hline $\mathrm{O}(4)-\mathrm{Ca}(2)-\mathrm{O}(2)$ & $124.49(9)$ & $\mathrm{O}(3)-\mathrm{C}(10)-\mathrm{C}(15)$ & $120.4(3)$ \\
\hline $\mathrm{O}(4)-\mathrm{Ca}(2)-\mathrm{O}(3) \# 1$ & $113.36(9)$ & $C(11)-C(10)-C(15)$ & $118.5(3)$ \\
\hline $\mathrm{O}(2)-\mathrm{Ca}(2)-\mathrm{O}(3) \# 1$ & $113.36(8)$ & $\mathrm{C}(12)-\mathrm{C}(11)-\mathrm{C}(10)$ & $119.3(3)$ \\
\hline $\mathrm{O}(4)-\mathrm{Ca}(2)-\mathrm{O}(1) \# 1$ & $85.90(8)$ & $C(12)-C(11)-C(16)$ & $120.2(3)$ \\
\hline $\mathrm{O}(2)-\mathrm{Ca}(2)-\mathrm{O}(1) \# 1$ & $77.53(7)$ & $C(10)-C(11)-C(16)$ & $120.4(3)$ \\
\hline $\mathrm{O}(3) \# 1-\mathrm{Ca}(2)-\mathrm{O}(1) \# 1$ & $76.51(7)$ & $C(11)-C(12)-C(13)$ & $122.8(3)$ \\
\hline $\mathrm{O}(4)-\mathrm{Ca}(2)-\mathrm{N}(3)$ & $77.83(9)$ & $\mathrm{C}(14)-\mathrm{C}(13)-\mathrm{C}(12)$ & 117.1(3) \\
\hline $\mathrm{O}(2)-\mathrm{Ca}(2)-\mathrm{N}(3)$ & $85.62(9)$ & $C(14)-C(13)-C(17)$ & $121.6(3)$ \\
\hline $\mathrm{O}(3) \# 1-\mathrm{Ca}(2)-\mathrm{N}(3)$ & $139.45(9)$ & $C(12)-C(13)-C(17)$ & $121.2(3)$ \\
\hline $\mathrm{O}(1) \# 1-\mathrm{Ca}(2)-\mathrm{N}(3)$ & 144.02(9) & $C(13)-C(14)-C(15)$ & $122.8(3)$ \\
\hline $\mathrm{O}(4)-\mathrm{Ca}(2)-\mathrm{N}(4)$ & $123.16(10)$ & $C(14)-C(15)-C(10)$ & $119.3(3)$ \\
\hline $\mathrm{O}(2)-\mathrm{Ca}(2)-\mathrm{N}(4)$ & $95.27(9)$ & $C(14)-C(15)-C(18)$ & $121.1(3)$ \\
\hline $\mathrm{O}(3) \# 1-\mathrm{Ca}(2)-\mathrm{N}(4)$ & $76.87(9)$ & $C(10)-C(15)-C(18)$ & $119.5(3)$ \\
\hline $\mathrm{O}(1) \# 1-\mathrm{Ca}(2)-\mathrm{N}(4)$ & $146.77(9)$ & $\mathrm{C}(19)-\mathrm{O}(4)-\mathrm{Ca}(2)$ & $141.2(2)$ \\
\hline $\mathrm{N}(3)-\mathrm{Ca}(2)-\mathrm{N}(4)$ & $65.52(10)$ & $\mathrm{O}(4)-\mathrm{C}(19)-\mathrm{C}(24)$ & $121.0(3)$ \\
\hline $\mathrm{C}(1)-\mathrm{O}(2)-\mathrm{Ca}(2)$ & $124.30(18)$ & $\mathrm{O}(4)-\mathrm{C}(19)-\mathrm{C}(20)$ & $120.6(3)$ \\
\hline $\mathrm{C}(1)-\mathrm{O}(2)-\mathrm{Ca}(1)$ & $132.79(19)$ & $\mathrm{C}(24)-\mathrm{C}(19)-\mathrm{C}(20)$ & $118.4(3)$ \\
\hline $\mathrm{Ca}(2)-\mathrm{O}(2)-\mathrm{Ca}(1)$ & $102.76(9)$ & $C(21)-C(20)-C(19)$ & $119.6(3)$ \\
\hline $\mathrm{O}(2)-\mathrm{C}(1)-\mathrm{C}(6)$ & $120.9(3)$ & $\mathrm{C}(21)-\mathrm{C}(20)-\mathrm{C}(25)$ & $120.9(3)$ \\
\hline $\mathrm{O}(2)-\mathrm{C}(1)-\mathrm{C}(2)$ & $121.4(3)$ & $\mathrm{C}(19)-\mathrm{C}(20)-\mathrm{C}(25)$ & $119.5(3)$ \\
\hline$C(6)-C(1)-C(2)$ & $117.7(3)$ & $\mathrm{C}(22)-\mathrm{C}(21)-\mathrm{C}(20)$ & $122.9(3)$ \\
\hline
\end{tabular}




$\begin{array}{llll}\mathrm{C}(21)-\mathrm{C}(22)-\mathrm{C}(23) & 116.6(3) & \mathrm{C}(31)-\mathrm{N}(4)-\mathrm{Ca}(2) & 114.2(2) \\ \mathrm{C}(21)-\mathrm{C}(22)-\mathrm{C}(26) & 121.1(3) & \mathrm{N}(3)-\mathrm{C}(30)-\mathrm{C}(31) & 109.8(3) \\ \mathrm{C}(23)-\mathrm{C}(22)-\mathrm{C}(26) & 122.3(3) & \mathrm{N}(4)-\mathrm{C}(31)-\mathrm{C}(30) & 109.5(3) \\ \mathrm{C}(24)-\mathrm{C}(23)-\mathrm{C}(22) & 123.2(3) & \mathrm{C}(37)-\mathrm{C}(32)-\mathrm{C}(33) & 117.8(4) \\ \mathrm{C}(23)-\mathrm{C}(24)-\mathrm{C}(19) & 119.4(3) & \mathrm{C}(37)-\mathrm{C}(32)-\mathrm{C}(38) & 121.2(4) \\ \mathrm{C}(23)-\mathrm{C}(24)-\mathrm{C}(27) & 121.2(3) & \mathrm{C}(33)-\mathrm{C}(32)-\mathrm{C}(38) & 121.1(4) \\ \mathrm{C}(19)-\mathrm{C}(24)-\mathrm{C}(27) & 119.4(3) & \mathrm{C}(34)-\mathrm{C}(33)-\mathrm{C}(32) & 121.2(4) \\ \mathrm{C}(28)-\mathrm{N}(1)-\mathrm{Ca}(1) & 109.1(2) & \mathrm{C}(33)-\mathrm{C}(34)-\mathrm{C}(35) & 120.1(5) \\ \mathrm{C}(29)-\mathrm{N}(2)-\mathrm{Ca}(1) & 110.8(2) & \mathrm{C}(36)-\mathrm{C}(35)-\mathrm{C}(34) & 119.9(5) \\ \mathrm{N}(1)-\mathrm{C}(28)-\mathrm{C}(29) & 110.8(3) & \mathrm{C}(35)-\mathrm{C}(36)-\mathrm{C}(37) & 119.8(4) \\ \mathrm{N}(2)-\mathrm{C}(29)-\mathrm{C}(28) & 110.7(3) & \mathrm{C}(32)-\mathrm{C}(37)-\mathrm{C}(36) & 121.2(4) \\ \mathrm{C}(30)-\mathrm{N}(3)-\mathrm{Ca}(2) & 112.7(2) & & \end{array}$

Symmetry transformations used to generate equivalent atoms:

$\# 1-x+2,-y+2,-z+1$ 
Table 4. Anisotropic displacement parameters $\left(\AA^{2} \times 10^{3}\right)$ for compound 3. The anisotropic displacement factor exponent takes the form: $-2 \pi^{2}\left[h^{2} a^{* 2} U^{11}+\ldots+2 h k^{*} b^{*} U^{12}\right]$

\begin{tabular}{|c|c|c|c|c|c|c|}
\hline & $\mathrm{U}^{11}$ & $\mathrm{U}^{22}$ & $\mathrm{U}^{33}$ & $\mathrm{U}^{23}$ & $\mathrm{U}^{13}$ & $\mathrm{U}^{12}$ \\
\hline $\mathrm{Ca}(1)$ & $17(1)$ & $15(1)$ & $17(1)$ & $5(1)$ & $3(1)$ & $4(1)$ \\
\hline $\mathrm{Ca}(2)$ & $17(1)$ & $18(1)$ & $18(1)$ & $8(1)$ & $6(1)$ & $8(1)$ \\
\hline $\mathrm{O}(1)$ & $17(1)$ & $19(1)$ & $17(1)$ & $7(1)$ & $0(1)$ & $6(1)$ \\
\hline $\mathrm{O}(2)$ & $23(1)$ & $27(1)$ & $18(1)$ & $10(1)$ & $4(1)$ & $7(1)$ \\
\hline $\mathrm{C}(1)$ & $16(2)$ & $27(2)$ & $23(2)$ & $14(2)$ & $6(1)$ & $2(1)$ \\
\hline $\mathrm{C}(2)$ & $18(2)$ & $28(2)$ & $30(2)$ & $16(2)$ & $7(2)$ & $4(2)$ \\
\hline$C(3)$ & $14(2)$ & $39(2)$ & $40(2)$ & $28(2)$ & $6(2)$ & $4(2)$ \\
\hline$C(4)$ & $24(2)$ & $39(2)$ & $27(2)$ & $21(2)$ & $1(2)$ & $-5(2)$ \\
\hline$C(5)$ & $29(2)$ & $23(2)$ & $21(2)$ & $6(2)$ & $3(2)$ & $-3(2)$ \\
\hline$C(6)$ & $22(2)$ & $24(2)$ & $21(2)$ & $11(2)$ & $6(1)$ & $5(2)$ \\
\hline$C(7)$ & $34(2)$ & $38(2)$ & $42(2)$ & $19(2)$ & $10(2)$ & $16(2)$ \\
\hline$C(8)$ & $41(3)$ & $53(3)$ & $38(3)$ & $27(2)$ & $-6(2)$ & $-6(2)$ \\
\hline$C(9)$ & $41(2)$ & $29(2)$ & $31(2)$ & $12(2)$ & $13(2)$ & $13(2)$ \\
\hline $\mathrm{O}(3)$ & $25(1)$ & $16(1)$ & $23(1)$ & $9(1)$ & $8(1)$ & $8(1)$ \\
\hline$C(10)$ & $27(2)$ & $18(2)$ & $12(2)$ & $6(1)$ & $1(1)$ & $8(1)$ \\
\hline $\mathrm{C}(11)$ & $24(2)$ & $21(2)$ & $18(2)$ & $8(2)$ & $3(1)$ & $9(2)$ \\
\hline $\mathrm{C}(12)$ & $27(2)$ & $29(2)$ & $28(2)$ & $14(2)$ & $4(2)$ & $15(2)$ \\
\hline$C(13)$ & $31(2)$ & $24(2)$ & $35(2)$ & $19(2)$ & $6(2)$ & $9(2)$ \\
\hline$C(14)$ & $24(2)$ & $26(2)$ & $30(2)$ & $16(2)$ & $6(2)$ & $4(2)$ \\
\hline$C(15)$ & $22(2)$ & $18(2)$ & $21(2)$ & $7(1)$ & $4(1)$ & $7(1)$ \\
\hline$C(16)$ & $29(2)$ & $22(2)$ & $31(2)$ & $11(2)$ & $6(2)$ & $10(2)$ \\
\hline$C(17)$ & $39(3)$ & $54(3)$ & $92(4)$ & $59(3)$ & $20(2)$ & $20(2)$ \\
\hline$C(18)$ & $25(2)$ & $27(2)$ & $37(2)$ & $14(2)$ & $11(2)$ & $10(2)$ \\
\hline $\mathrm{O}(4)$ & $29(1)$ & $24(1)$ & $32(1)$ & $10(1)$ & $11(1)$ & $17(1)$ \\
\hline$C(19)$ & $22(2)$ & $25(2)$ & $25(2)$ & $11(2)$ & $3(2)$ & $11(2)$ \\
\hline$C(20)$ & $26(2)$ & $21(2)$ & $35(2)$ & $11(2)$ & $11(2)$ & $11(2)$ \\
\hline$C(21)$ & $23(2)$ & $31(2)$ & $35(2)$ & $15(2)$ & $12(2)$ & $11(2)$ \\
\hline $\mathrm{C}(22)$ & $26(2)$ & $26(2)$ & $22(2)$ & $6(2)$ & $2(2)$ & $12(2)$ \\
\hline$C(23)$ & $25(2)$ & $19(2)$ & $28(2)$ & $7(2)$ & $4(2)$ & $7(2)$ \\
\hline$C(24)$ & $25(2)$ & $25(2)$ & $19(2)$ & $8(2)$ & $3(1)$ & $11(2)$ \\
\hline$C(25)$ & $42(2)$ & $33(2)$ & $71(3)$ & $28(2)$ & $36(2)$ & $18(2)$ \\
\hline
\end{tabular}




\begin{tabular}{lllllll}
$\mathrm{C}(26)$ & $37(2)$ & $32(2)$ & $32(2)$ & $9(2)$ & $11(2)$ & $19(2)$ \\
$\mathrm{C}(27)$ & $33(2)$ & $30(2)$ & $34(2)$ & $16(2)$ & $13(2)$ & $18(2)$ \\
$\mathrm{N}(1)$ & $30(2)$ & $17(2)$ & $22(2)$ & $1(1)$ & $2(1)$ & $8(1)$ \\
$\mathrm{N}(2)$ & $18(2)$ & $28(2)$ & $33(2)$ & $8(1)$ & $7(1)$ & $8(1)$ \\
$\mathrm{C}(28)$ & $29(2)$ & $22(2)$ & $25(2)$ & $4(2)$ & $-1(2)$ & $1(2)$ \\
$\mathrm{C}(29)$ & $20(2)$ & $35(2)$ & $30(2)$ & $14(2)$ & $3(2)$ & $2(2)$ \\
$\mathrm{N}(3)$ & $39(2)$ & $36(2)$ & $25(2)$ & $14(2)$ & $16(1)$ & $20(2)$ \\
$\mathrm{N}(4)$ & $47(2)$ & $39(2)$ & $24(2)$ & $16(2)$ & $3(2)$ & $24(2)$ \\
$\mathrm{C}(30)$ & $30(2)$ & $38(2)$ & $31(2)$ & $18(2)$ & $9(2)$ & $5(2)$ \\
$\mathrm{C}(31)$ & $31(2)$ & $35(2)$ & $26(2)$ & $19(2)$ & $1(2)$ & $5(2)$ \\
$\mathrm{C}(32)$ & $45(3)$ & $26(2)$ & $39(3)$ & $10(2)$ & $2(2)$ & $4(2)$ \\
$\mathrm{C}(33)$ & $62(3)$ & $49(3)$ & $38(3)$ & $20(2)$ & $0(2)$ & $13(2)$ \\
$\mathrm{C}(34)$ & $45(3)$ & $77(4)$ & $45(3)$ & $17(3)$ & $3(2)$ & $23(3)$ \\
$\mathrm{C}(35)$ & $56(3)$ & $71(4)$ & $46(3)$ & $22(3)$ & $19(3)$ & $23(3)$ \\
$\mathrm{C}(36)$ & $63(3)$ & $51(3)$ & $35(2)$ & $17(2)$ & $11(2)$ & $31(2)$ \\
$\mathrm{C}(37)$ & $51(3)$ & $32(2)$ & $37(2)$ & $7(2)$ & $2(2)$ & $15(2)$ \\
$\mathrm{C}(38)$ & $57(3)$ & $57(3)$ & $64(4)$ & $27(3)$ & $15(3)$ & $0(3)$ \\
& & & & & & \\
\hline
\end{tabular}


Table 5. Hydrogen coordinates $\left(\times 10^{4}\right)$ and isotropic displacement parameters $\left(\AA^{2} \times 10^{3}\right)$ for compound 3.

\begin{tabular}{|c|c|c|c|c|}
\hline & $\mathrm{x}$ & $\mathrm{y}$ & $\mathrm{z}$ & $\mathrm{U}(\mathrm{eq})$ \\
\hline $\mathrm{H}(1 \mathrm{~A})$ & 9572 & 10572 & 5936 & 28 \\
\hline $\mathrm{H}(3 \mathrm{~A})$ & 6919 & 8953 & 536 & 35 \\
\hline $\mathrm{H}(5 \mathrm{~A})$ & 7831 & 6206 & -622 & 37 \\
\hline $\mathrm{H}(7 \mathrm{~A})$ & 7504 & 10358 & 2258 & 56 \\
\hline $\mathrm{H}(7 \mathrm{~B})$ & 8797 & 10628 & 2767 & 56 \\
\hline $\mathrm{H}(7 \mathrm{C})$ & 7668 & 9866 & 3016 & 56 \\
\hline $\mathrm{H}(8 \mathrm{~A})$ & 6140 & 7567 & -1211 & 74 \\
\hline $\mathrm{H}(8 \mathrm{~B})$ & 5894 & 6259 & -1452 & 74 \\
\hline $\mathrm{H}(8 \mathrm{C})$ & 7012 & 6973 & -1747 & 74 \\
\hline $\mathrm{H}(9 \mathrm{~A})$ & 8986 & 5878 & 365 & 52 \\
\hline $\mathrm{H}(9 \mathrm{~B})$ & 8837 & 6338 & 1526 & 52 \\
\hline $\mathrm{H}(9 \mathrm{C})$ & 9982 & 7053 & 1256 & 52 \\
\hline $\mathrm{H}(12 \mathrm{~A})$ & 8974 & 5150 & 5348 & 32 \\
\hline $\mathrm{H}(14 \mathrm{~A})$ & 5892 & 5359 & 5923 & 33 \\
\hline $\mathrm{H}(16 \mathrm{~A})$ & 10397 & 6473 & 5007 & 42 \\
\hline $\mathrm{H}(16 \mathrm{~B})$ & 10481 & 7784 & 5642 & 42 \\
\hline $\mathrm{H}(16 \mathrm{C})$ & 9895 & 7027 & 4448 & 42 \\
\hline $\mathrm{H}(17 \mathrm{~A})$ & 7649 & 3701 & 5664 & 77 \\
\hline $\mathrm{H}(17 \mathrm{~B})$ & 6297 & 3379 & 5267 & 77 \\
\hline $\mathrm{H}(17 \mathrm{C})$ & 6872 & 4166 & 6462 & 77 \\
\hline $\mathrm{H}(18 \mathrm{~A})$ & 5435 & 6910 & 6040 & 45 \\
\hline $\mathrm{H}(18 \mathrm{~B})$ & 5978 & 7413 & 5299 & 45 \\
\hline $\mathrm{H}(18 \mathrm{C})$ & 6563 & 8122 & 6492 & 45 \\
\hline $\mathrm{H}(21 \mathrm{~A})$ & 15051 & 8271 & 1103 & 36 \\
\hline $\mathrm{H}(23 \mathrm{~A})$ & 12687 & 5919 & 1694 & 32 \\
\hline $\mathrm{H}(25 \mathrm{~A})$ & 15211 & 10207 & 1784 & 69 \\
\hline $\mathrm{H}(25 \mathrm{~B})$ & 14942 & 10707 & 2908 & 69 \\
\hline $\mathrm{H}(25 \mathrm{C})$ & 14001 & 10368 & 1954 & 69 \\
\hline $\mathrm{H}(26 \mathrm{~A})$ & 15029 & 6394 & 344 & 53 \\
\hline $\mathrm{H}(26 \mathrm{~B})$ & 13744 & 5388 & 48 & 53 \\
\hline
\end{tabular}




\begin{tabular}{|c|c|c|c|c|}
\hline $\mathrm{H}(26 \mathrm{C})$ & 14674 & 5750 & 1019 & 53 \\
\hline $\mathrm{H}(27 \mathrm{~A})$ & 11367 & 6372 & 2741 & 45 \\
\hline $\mathrm{H}(27 \mathrm{~B})$ & 10957 & 7329 & 2705 & 45 \\
\hline $\mathrm{H}(27 \mathrm{C})$ & 11912 & 7690 & 3659 & 45 \\
\hline $\mathrm{H}(1 \mathrm{~B})$ & 8149 & 6836 & 3698 & 33 \\
\hline $\mathrm{H}(1 \mathrm{C})$ & 8444 & 5924 & 2822 & 33 \\
\hline $\mathrm{H}(2 \mathrm{~A})$ & 7392 & 8018 & 3095 & 36 \\
\hline $\mathrm{H}(2 \mathrm{~B})$ & 6407 & 8283 & 3612 & 36 \\
\hline $\mathrm{H}(28 \mathrm{~A})$ & 6354 & 5007 & 2207 & 38 \\
\hline $\mathrm{H}(28 \mathrm{~B})$ & 6924 & 5928 & 1806 & 38 \\
\hline $\mathrm{H}(29 \mathrm{~A})$ & 5319 & 6213 & 2577 & 38 \\
\hline $\mathrm{H}(29 \mathrm{~B})$ & 6010 & 6478 & 3634 & 38 \\
\hline $\mathrm{H}(3 \mathrm{~B})$ & 10735 & 9034 & 1584 & 38 \\
\hline $\mathrm{H}(3 \mathrm{C})$ & 11660 & 8622 & 1077 & 38 \\
\hline $\mathrm{H}(4 \mathrm{~A})$ & 11533 & 11189 & 2654 & 41 \\
\hline $\mathrm{H}(4 \mathrm{~B})$ & 10626 & 11685 & 2609 & 41 \\
\hline $\mathrm{H}(30 \mathrm{~A})$ & 11408 & 9524 & 86 & 42 \\
\hline $\mathrm{H}(30 \mathrm{~B})$ & 12387 & 10481 & 1110 & 42 \\
\hline $\mathrm{H}(31 \mathrm{~A})$ & 10949 & 11180 & 916 & 38 \\
\hline $\mathrm{H}(31 \mathrm{~B})$ & 9941 & 10076 & 910 & 38 \\
\hline $\mathrm{H}(33 \mathrm{~A})$ & 1170 & 6377 & 6697 & 63 \\
\hline $\mathrm{H}(34 \mathrm{~A})$ & -350 & 6049 & 7521 & 73 \\
\hline $\mathrm{H}(35 \mathrm{~A})$ & 5 & 6198 & 9126 & 73 \\
\hline $\mathrm{H}(36 \mathrm{~A})$ & 1894 & 6695 & 9898 & 59 \\
\hline $\mathrm{H}(37 \mathrm{~A})$ & 3436 & 7030 & 9067 & 53 \\
\hline $\mathrm{H}(38 \mathrm{~A})$ & 4191 & 7058 & 7646 & 100 \\
\hline $\mathrm{H}(38 \mathrm{~B})$ & 3544 & 7500 & 7065 & 100 \\
\hline $\mathrm{H}(38 \mathrm{C})$ & 3273 & 6136 & 6583 & 100 \\
\hline
\end{tabular}


Table for Compound 4: $\left[\mathbf{C a}_{2}\left(\eta^{2}-{ }^{t} \mathbf{B u} \mathbf{u}_{2} \mathbf{p z}\right)\left(\mu-\eta^{1}: \eta^{2}-{ }^{t} \mathbf{B} \mathbf{u}_{2} \mathbf{p z}\right)\left(\mu-\eta^{2}: \eta^{4}-{ }^{t} \mathbf{B u} \mathbf{u}_{2} \mathbf{p z}\right)(\mathrm{OH})(\text { thf })\right]_{2}$ 
Table 1. Crystal data and structure refinement for compound 4.

Identification code

Empirical formula

Formula weight

Temperature

Wavelength

Crystal system

Space group

Unit cell dimensions

Volume

Z

Density (calculated)

Absorption coefficient

$\mathrm{F}(000)$

Crystal size

Theta range for data collection

Index ranges

Reflections collected

Independent reflections

Completeness to theta $=25.00^{\circ}$

Absorption correction

Max. and min. transmission

Refinement method

Data / restraints / parameters

Goodness-of-fit on $\mathrm{F}^{2}$

Final $R$ indices [I $>2 \operatorname{sigma}(\mathrm{I})]$

$\mathrm{R}$ indices (all data)

Largest diff. peak and hole p-1

C74 H132 Ca4 N12 O4

1414.24

94(2) K

$0.71073 \AA$

Triclinic

P-1

$\mathrm{a}=13.816(2) \AA$

$\alpha=76.093(3)^{\circ}$.

$\mathrm{b}=14.250(2) \AA$

$\beta=75.236(3)^{\circ}$.

$\mathrm{c}=25.075(4) \AA$

$\gamma=64.205(3)^{\circ}$.

4250.4(11) $\AA^{3}$

2

$1.105 \mathrm{Mg} / \mathrm{m}^{3}$

$0.304 \mathrm{~mm}^{-1}$

1544

$0.20 \times 0.14 \times 0.04 \mathrm{~mm}^{3}$

1.66 to $25.00^{\circ}$.

$-16<=\mathrm{h}<=16,-16<=\mathrm{k}<=16,-29<=\mathrm{l}<=29$

34786

$14969[\mathrm{R}(\mathrm{int})=0.0942]$

$99.9 \%$

Semi-empirical from equivalents

0.9879 and 0.9417

Full-matrix least-squares on $\mathrm{F}^{2}$

14969 / 0 / 891

0.997

$\mathrm{R} 1=0.0748, \mathrm{wR} 2=0.1683$

$\mathrm{R} 1=0.1512, \mathrm{wR} 2=0.2043$

1.127 and -0.587 e. $\AA^{-3}$ 
Table 2. Atomic coordinates $\left(\times 10^{4}\right)$ and equivalent isotropic displacement parameters $\left(\AA^{2} \times 10^{3}\right)$ for compound 4. $U(e q)$ is defined as one third of the trace of the orthogonalized $U^{i j}$ tensor.

\begin{tabular}{|c|c|c|c|c|}
\hline & $\mathrm{x}$ & $\mathrm{y}$ & $\mathrm{z}$ & $\mathrm{U}(\mathrm{eq})$ \\
\hline $\mathrm{Ca}(1)$ & 4097(1) & $4386(1)$ & $5060(1)$ & $22(1)$ \\
\hline $\mathrm{O}(1)$ & $4683(3)$ & $5577(3)$ & $4454(2)$ & $21(1)$ \\
\hline $\mathrm{Ca}(2)$ & $6676(1)$ & $2742(1)$ & $5539(1)$ & $22(1)$ \\
\hline $\mathrm{O}(2)$ & $-1132(3)$ & $814(3)$ & 10041(2) & $23(1)$ \\
\hline $\mathrm{Ca}(3)$ & $487(1)$ & 1011(1) & $9843(1)$ & $27(1)$ \\
\hline $\mathrm{Ca}(4)$ & $-2066(1)$ & 1181(1) & 10921(1) & $30(1)$ \\
\hline $\mathrm{N}(11)$ & 1967(3) & $6546(3)$ & $4883(2)$ & $23(1)$ \\
\hline $\mathrm{N}(12)$ & $2333(3)$ & $5767(3)$ & $5330(2)$ & $22(1)$ \\
\hline$C(13)$ & $1450(4)$ & $5668(4)$ & $5683(2)$ & $26(1)$ \\
\hline$C(14)$ & $511(4)$ & $6371(4)$ & $5469(2)$ & $27(1)$ \\
\hline$C(15)$ & $854(4)$ & $6903(4)$ & 4972(2) & $23(1)$ \\
\hline $\mathrm{N}(21)$ & $5673(3)$ & $2792(3)$ & $4812(2)$ & $23(1)$ \\
\hline $\mathrm{N}(22)$ & $5049(3)$ & $2507(3)$ & $5310(2)$ & $24(1)$ \\
\hline$C(23)$ & $5390(4)$ & $1453(3)$ & $5387(2)$ & $22(1)$ \\
\hline$C(24)$ & $6231(4)$ & 1044(4) & 4954(2) & $26(1)$ \\
\hline$C(25)$ & $6384(3)$ & 1903(3) & $4600(2)$ & $21(1)$ \\
\hline $\mathrm{N}(31)$ & $6639(3)$ & $2399(3)$ & $6478(2)$ & $24(1)$ \\
\hline $\mathrm{N}(32)$ & $7227(3)$ & $1415(3)$ & $6305(2)$ & $23(1)$ \\
\hline$C(33)$ & $7503(4)$ & $717(4)$ & $6761(2)$ & $23(1)$ \\
\hline$C(34)$ & 7111(4) & $1229(4)$ & $7226(2)$ & $27(1)$ \\
\hline$C(35)$ & 6571(4) & $2275(4)$ & $7029(2)$ & $25(1)$ \\
\hline $\mathrm{O}(40)$ & $3205(3)$ & $3888(3)$ & $4531(2)$ & $35(1)$ \\
\hline$C(41)$ & $3605(5)$ & $2908(4)$ & $4326(2)$ & $41(1)$ \\
\hline$C(42)$ & $3337(6)$ & $3178(5)$ & $3776(3)$ & $63(2)$ \\
\hline$C(43)$ & $2308(4)$ & $4152(4)$ & 3791(2) & $40(1)$ \\
\hline$C(44)$ & $2228(5)$ & $4559(4)$ & $4310(3)$ & $47(2)$ \\
\hline $\mathrm{N}(51)$ & $934(3)$ & 791(3) & $8848(2)$ & $32(1)$ \\
\hline $\mathrm{N}(52)$ & $1878(3)$ & $736(3)$ & 8993(2) & $34(1)$ \\
\hline$C(52)$ & 6716(4) & $3013(4)$ & $3710(2)$ & $36(1)$ \\
\hline$C(53)$ & 2501(5) & $960(4)$ & $8517(2)$ & $42(2)$ \\
\hline $\mathrm{C}(54)$ & $1988(6)$ & $1151(5)$ & $8075(2)$ & $56(2)$ \\
\hline
\end{tabular}




\begin{tabular}{|c|c|c|c|c|}
\hline $\mathrm{C}(55)$ & $1003(5)$ & $1057(4)$ & $8291(2)$ & $39(1)$ \\
\hline $\mathrm{N}(61)$ & 161(3) & 1503(3) & $10767(2)$ & $28(1)$ \\
\hline $\mathrm{N}(62)$ & $-914(3)$ & 1976(3) & $11029(2)$ & $30(1)$ \\
\hline$C(63)$ & $-922(4)$ & 2592(4) & $11373(2)$ & $33(1)$ \\
\hline$C(64)$ & $123(4)$ & $2517(4)$ & $11333(2)$ & $35(1)$ \\
\hline$C(65)$ & $773(4)$ & $1822(4)$ & $10955(2)$ & $28(1)$ \\
\hline $\mathrm{N}(71)$ & $-3942(3)$ & $2280(3)$ & $11139(2)$ & $28(1)$ \\
\hline $\mathrm{N}(72)$ & $-3615(3)$ & $2359(3)$ & $10566(2)$ & $31(1)$ \\
\hline$C(73)$ & $-4485(4)$ & 2972(4) & $10328(2)$ & $35(1)$ \\
\hline $\mathrm{C}(74)$ & $-5393(4)$ & $3308(4)$ & $10738(2)$ & $35(1)$ \\
\hline$C(75)$ & $-5018(4)$ & 2853(4) & $11237(2)$ & $30(1)$ \\
\hline $\mathrm{O}(80)$ & $-98(3)$ & 2864(3) & $9532(2)$ & $46(1)$ \\
\hline $\mathrm{C}(81)$ & $-786(6)$ & $3652(5)$ & $9888(3)$ & $71(2)$ \\
\hline $\mathrm{C}(82)$ & $-1041(8)$ & $4677(6)$ & 9513(3) & 107(3) \\
\hline $\mathrm{C}(83)$ & $-158(18)$ & $4423(12)$ & $9003(7)$ & $79(7)$ \\
\hline $\mathrm{C}(84)$ & $56(7)$ & $3370(5)$ & 8977(3) & $72(2)$ \\
\hline$C(85)$ & $-771(18)$ & $4413(12)$ & $8911(6)$ & $62(7)$ \\
\hline$C(131)$ & $1530(4)$ & 4921(4) & $6221(2)$ & $30(1)$ \\
\hline$C(132)$ & $2692(4)$ & $4274(4)$ & $6312(2)$ & $37(1)$ \\
\hline$C(133)$ & $995(4)$ & $4170(4)$ & $6231(3)$ & $45(2)$ \\
\hline$C(134)$ & $905(5)$ & $5564(4)$ & $6701(2)$ & $47(2)$ \\
\hline$C(151)$ & $183(4)$ & $7760(4)$ & $4564(2)$ & $27(1)$ \\
\hline$C(152)$ & $211(4)$ & $8810(4)$ & $4587(2)$ & $36(1)$ \\
\hline$C(153)$ & $613(4)$ & $7511(4)$ & $3972(2)$ & $36(1)$ \\
\hline$C(154)$ & $-1010(4)$ & 7873(4) & $4736(2)$ & $41(1)$ \\
\hline$C(231)$ & $4796(4)$ & $917(4)$ & $5872(2)$ & $28(1)$ \\
\hline$C(232)$ & $5376(4)$ & $-286(4)$ & $5896(2)$ & $34(1)$ \\
\hline$C(233)$ & $4773(4)$ & $1214(4)$ & $6424(2)$ & $33(1)$ \\
\hline$C(234)$ & $3623(4)$ & 1273(4) & $5777(2)$ & $35(1)$ \\
\hline$C(251)$ & $7175(4)$ & 1951(4) & $4069(2)$ & $25(1)$ \\
\hline$C(253)$ & $7379(4)$ & 1076(4) & $3746(2)$ & $38(1)$ \\
\hline$C(254)$ & $8266(4)$ & 1792(4) & $4200(2)$ & $36(1)$ \\
\hline$C(331)$ & $8127(4)$ & $-460(4)$ & $6742(2)$ & $27(1)$ \\
\hline$C(332)$ & $7404(5)$ & $-1044(4)$ & $7076(2)$ & $41(1)$ \\
\hline$C(333)$ & $9151(4)$ & $-862(4)$ & $7003(2)$ & $39(1)$ \\
\hline$C(334)$ & $8461(4)$ & $-702(4)$ & $6143(2)$ & $33(1)$ \\
\hline
\end{tabular}




\begin{tabular}{|c|c|c|c|c|}
\hline$C(351)$ & $5956(4)$ & $3247(4)$ & $7327(2)$ & $33(1)$ \\
\hline$C(352)$ & $6395(7)$ & $4053(5)$ & 7047(3) & $91(3)$ \\
\hline$C(353)$ & $4745(5)$ & $3701(5)$ & 7264(3) & $74(2)$ \\
\hline$C(354)$ & $5987(6)$ & $2953(5)$ & 7931(3) & $69(2)$ \\
\hline$C(531)$ & $3575(5)$ & $1028(5)$ & $8533(3)$ & $51(2)$ \\
\hline$C(532)$ & $3279(6)$ & $2079(6)$ & $8737(3)$ & $71(2)$ \\
\hline$C(533)$ & $4199(5)$ & $110(6)$ & $8939(3)$ & $63(2)$ \\
\hline$C(534)$ & $4246(6)$ & $1031(6)$ & $7944(3)$ & $77(2)$ \\
\hline$C(551)$ & $113(5)$ & $1216(5)$ & $7995(2)$ & $45(2)$ \\
\hline$C(552)$ & $463(8)$ & $322(7)$ & $7674(4)$ & $123(4)$ \\
\hline$C(553)$ & $-206(7)$ & $2271(6)$ & $7618(3)$ & $92(3)$ \\
\hline$C(554)$ & $-910(6)$ & $1237(7)$ & $8417(3)$ & $84(3)$ \\
\hline$C(631)$ & $-1955(5)$ & $3256(4)$ & $11712(2)$ & $43(2)$ \\
\hline$C(632)$ & $-1694(5)$ & $3841(5)$ & $12059(3)$ & $66(2)$ \\
\hline$C(633)$ & $-2501(5)$ & $2559(5)$ & $12119(3)$ & $56(2)$ \\
\hline$C(634)$ & $-2735(4)$ & $4062(4)$ & $11330(2)$ & $49(2)$ \\
\hline$C(651)$ & $2000(4)$ & $1416(4)$ & $10799(2)$ & $37(1)$ \\
\hline$C(652)$ & 2423(4) & $763(5)$ & $10332(2)$ & $47(2)$ \\
\hline$C(653)$ & $2365(5)$ & $2326(5)$ & $10617(3)$ & $62(2)$ \\
\hline$C(654)$ & $2484(5)$ & $740(6)$ & $11315(3)$ & $68(2)$ \\
\hline$C(731)$ & $-4383(5)$ & $3163(4)$ & $9699(2)$ & $45(2)$ \\
\hline$C(732)$ & $-3447(5)$ & $3498(5)$ & $9425(2)$ & $61(2)$ \\
\hline$C(733)$ & $-4153(6)$ & $2130(5)$ & $9512(3)$ & $61(2)$ \\
\hline$C(734)$ & $-5433(6)$ & $4016(6)$ & $9519(3)$ & $81(3)$ \\
\hline$C(751)$ & $-5659(4)$ & $2986(4)$ & $11818(2)$ & $35(1)$ \\
\hline$C(752)$ & $-5729(6)$ & $3982(5)$ & 11980(3) & $58(2)$ \\
\hline$C(753)$ & $-5150(5)$ & $2041(5)$ & $12246(2)$ & $56(2)$ \\
\hline$C(754)$ & $-6827(5)$ & $3118(5)$ & $11834(3)$ & $59(2)$ \\
\hline
\end{tabular}


Table 3. Bond lengths $[\AA]]$ and angles $\left[^{\circ}\right]$ for compound 4.

\begin{tabular}{|c|c|c|c|}
\hline $\mathrm{Ca}(1)-\mathrm{O}(1)$ & $2.276(4)$ & $\mathrm{N}(12)-\mathrm{Ca}(2) \# 1$ & $3.151(4)$ \\
\hline $\mathrm{Ca}(1)-\mathrm{O}(1) \# 1$ & $2.342(4)$ & $\mathrm{C}(13)-\mathrm{C}(14)$ & $1.393(6)$ \\
\hline $\mathrm{Ca}(1)-\mathrm{O}(40)$ & $2.416(3)$ & $C(13)-C(131)$ & $1.501(7)$ \\
\hline $\mathrm{Ca}(1)-\mathrm{N}(22)$ & $2.416(4)$ & $C(14)-C(15)$ & $1.381(7)$ \\
\hline $\mathrm{Ca}(1)-\mathrm{N}(12)$ & $2.429(4)$ & $C(15)-C(151)$ & $1.514(6)$ \\
\hline $\mathrm{Ca}(1)-\mathrm{N}(21)$ & $2.444(4)$ & $\mathrm{N}(21)-\mathrm{C}(25)$ & $1.351(5)$ \\
\hline $\mathrm{O}(1)-\mathrm{Ca}(2) \# 1$ & $2.313(3)$ & $\mathrm{N}(21)-\mathrm{N}(22)$ & $1.406(5)$ \\
\hline $\mathrm{O}(1)-\mathrm{Ca}(1) \# 1$ & $2.342(4)$ & $\mathrm{N}(22)-\mathrm{C}(23)$ & $1.344(6)$ \\
\hline $\mathrm{Ca}(2)-\mathrm{N}(31)$ & $2.278(4)$ & $\mathrm{C}(23)-\mathrm{C}(24)$ & $1.393(6)$ \\
\hline $\mathrm{Ca}(2)-\mathrm{O}(1) \# 1$ & $2.313(3)$ & $\mathrm{C}(23)-\mathrm{C}(231)$ & $1.525(6)$ \\
\hline $\mathrm{Ca}(2)-\mathrm{N}(32)$ & $2.364(4)$ & $C(24)-C(25)$ & $1.393(6)$ \\
\hline $\mathrm{Ca}(2)-\mathrm{N}(11) \# 1$ & $2.407(4)$ & $\mathrm{C}(25)-\mathrm{C}(251)$ & $1.499(6)$ \\
\hline $\mathrm{Ca}(2)-\mathrm{N}(21)$ & $2.528(4)$ & $\mathrm{N}(31)-\mathrm{C}(35)$ & $1.334(6)$ \\
\hline $\mathrm{Ca}(2)-\mathrm{N}(22)$ & $2.627(4)$ & $\mathrm{N}(31)-\mathrm{N}(32)$ & $1.392(5)$ \\
\hline $\mathrm{Ca}(2)-\mathrm{C}(25)$ & $3.065(5)$ & $\mathrm{N}(32)-\mathrm{C}(33)$ & $1.337(6)$ \\
\hline $\mathrm{Ca}(2)-\mathrm{N}(12) \# 1$ & $3.151(4)$ & $\mathrm{C}(33)-\mathrm{C}(34)$ & $1.388(6)$ \\
\hline $\mathrm{Ca}(2)-\mathrm{C}(23)$ & $3.193(4)$ & $\mathrm{C}(33)-\mathrm{C}(331)$ & $1.523(6)$ \\
\hline $\mathrm{O}(2)-\mathrm{Ca}(3)$ & $2.289(4)$ & $\mathrm{C}(34)-\mathrm{C}(35)$ & $1.375(6)$ \\
\hline $\mathrm{O}(2)-\mathrm{Ca}(4)$ & $2.308(4)$ & $\mathrm{C}(35)-\mathrm{C}(351)$ & $1.533(7)$ \\
\hline $\mathrm{O}(2)-\mathrm{Ca}(3) \# 2$ & $2.323(4)$ & $\mathrm{O}(40)-\mathrm{C}(44)$ & $1.430(6)$ \\
\hline $\mathrm{Ca}(3)-\mathrm{O}(2) \# 2$ & $2.323(4)$ & $\mathrm{O}(40)-\mathrm{C}(41)$ & $1.432(6)$ \\
\hline $\mathrm{Ca}(3)-\mathrm{O}(80)$ & $2.389(4)$ & $\mathrm{C}(41)-\mathrm{C}(42)$ & $1.438(8)$ \\
\hline $\mathrm{Ca}(3)-\mathrm{N}(61)$ & $2.457(4)$ & $\mathrm{C}(42)-\mathrm{C}(43)$ & $1.493(8)$ \\
\hline $\mathrm{Ca}(3)-\mathrm{N}(52)$ & $2.458(4)$ & $\mathrm{C}(43)-\mathrm{C}(44)$ & $1.509(7)$ \\
\hline $\mathrm{Ca}(3)-\mathrm{N}(51)$ & $2.475(4)$ & $\mathrm{N}(51)-\mathrm{C}(55)$ & $1.345(6)$ \\
\hline $\mathrm{Ca}(4)-\mathrm{N}(72)$ & $2.306(4)$ & $\mathrm{N}(51)-\mathrm{N}(52)$ & $1.406(6)$ \\
\hline $\mathrm{Ca}(4)-\mathrm{N}(71)$ & $2.372(4)$ & $\mathrm{N}(51)-\mathrm{Ca}(4) \# 2$ & $2.556(4)$ \\
\hline $\mathrm{Ca}(4)-\mathrm{N}(62)$ & $2.413(4)$ & $\mathrm{N}(52)-\mathrm{C}(53)$ & $1.342(6)$ \\
\hline $\mathrm{Ca}(4)-\mathrm{N}(51) \# 2$ & $2.556(4)$ & $\mathrm{N}(52)-\mathrm{Ca}(4) \# 2$ & $2.589(4)$ \\
\hline $\mathrm{Ca}(4)-\mathrm{N}(52) \# 2$ & $2.589(4)$ & $\mathrm{C}(52)-\mathrm{C}(251)$ & $1.518(7)$ \\
\hline $\mathrm{N}(11)-\mathrm{C}(15)$ & $1.369(6)$ & $\mathrm{C}(53)-\mathrm{C}(54)$ & $1.374(8)$ \\
\hline $\mathrm{N}(11)-\mathrm{N}(12)$ & $1.395(5)$ & $\mathrm{C}(53)-\mathrm{C}(531)$ & $1.541(8)$ \\
\hline $\mathrm{N}(11)-\mathrm{Ca}(2) \# 1$ & $2.407(4)$ & $\mathrm{C}(54)-\mathrm{C}(55)$ & $1.382(8)$ \\
\hline $\mathrm{N}(12)-\mathrm{C}(13)$ & $1.352(6)$ & $\mathrm{C}(55)-\mathrm{C}(551)$ & $1.503(8)$ \\
\hline
\end{tabular}




\begin{tabular}{|c|c|c|c|}
\hline $\mathrm{N}(61)-\mathrm{C}(65)$ & $1.336(6)$ & $\mathrm{C}(231)-\mathrm{C}(232)$ & $1.538(6)$ \\
\hline $\mathrm{N}(61)-\mathrm{N}(62)$ & $1.391(5)$ & $\mathrm{C}(231)-\mathrm{C}(234)$ & $1.538(7)$ \\
\hline $\mathrm{N}(62)-\mathrm{C}(63)$ & $1.367(6)$ & $\mathrm{C}(251)-\mathrm{C}(254)$ & $1.534(7)$ \\
\hline$C(63)-C(64)$ & $1.379(7)$ & $\mathrm{C}(251)-\mathrm{C}(253)$ & $1.534(6)$ \\
\hline $\mathrm{C}(63)-\mathrm{C}(631)$ & $1.500(7)$ & $\mathrm{C}(331)-\mathrm{C}(334)$ & $1.531(7)$ \\
\hline$C(64)-C(65)$ & $1.389(7)$ & $\mathrm{C}(331)-\mathrm{C}(333)$ & $1.532(7)$ \\
\hline $\mathrm{C}(65)-\mathrm{C}(651)$ & $1.510(7)$ & $\mathrm{C}(331)-\mathrm{C}(332)$ & $1.534(7)$ \\
\hline $\mathrm{N}(71)-\mathrm{C}(75)$ & $1.339(6)$ & $\mathrm{C}(351)-\mathrm{C}(352)$ & $1.477(8)$ \\
\hline $\mathrm{N}(71)-\mathrm{N}(72)$ & $1.384(5)$ & $\mathrm{C}(351)-\mathrm{C}(354)$ & $1.478(8)$ \\
\hline $\mathrm{N}(72)-\mathrm{C}(73)$ & $1.329(6)$ & $\mathrm{C}(351)-\mathrm{C}(353)$ & $1.545(8)$ \\
\hline$C(73)-C(74)$ & $1.384(7)$ & $\mathrm{C}(531)-\mathrm{C}(533)$ & $1.523(9)$ \\
\hline$C(73)-C(731)$ & $1.515(7)$ & $\mathrm{C}(531)-\mathrm{C}(534)$ & $1.530(8)$ \\
\hline$C(74)-C(75)$ & $1.380(7)$ & $C(531)-C(532)$ & $1.549(8)$ \\
\hline$C(75)-C(751)$ & $1.507(7)$ & $\mathrm{C}(551)-\mathrm{C}(552)$ & $1.504(9)$ \\
\hline $\mathrm{O}(80)-\mathrm{C}(84)$ & $1.415(7)$ & $\mathrm{C}(551)-\mathrm{C}(553)$ & $1.516(8)$ \\
\hline $\mathrm{O}(80)-\mathrm{C}(81)$ & $1.442(7)$ & $\mathrm{C}(551)-\mathrm{C}(554)$ & $1.528(9)$ \\
\hline $\mathrm{C}(81)-\mathrm{C}(82)$ & $1.484(9)$ & $\mathrm{C}(631)-\mathrm{C}(634)$ & $1.522(8)$ \\
\hline $\mathrm{C}(82)-\mathrm{C}(83)$ & $1.517(16)$ & $\mathrm{C}(631)-\mathrm{C}(632)$ & $1.533(8)$ \\
\hline $\mathrm{C}(82)-\mathrm{C}(85)$ & $1.559(17)$ & $\mathrm{C}(631)-\mathrm{C}(633)$ & $1.539(8)$ \\
\hline $\mathrm{C}(83)-\mathrm{C}(84)$ & $1.412(16)$ & $\mathrm{C}(651)-\mathrm{C}(652)$ & $1.510(7)$ \\
\hline $\mathrm{C}(84)-\mathrm{C}(85)$ & $1.430(16)$ & $\mathrm{C}(651)-\mathrm{C}(653)$ & $1.519(8)$ \\
\hline $\mathrm{C}(131)-\mathrm{C}(132)$ & $1.508(7)$ & $\mathrm{C}(651)-\mathrm{C}(654)$ & $1.526(8)$ \\
\hline $\mathrm{C}(131)-\mathrm{C}(134)$ & $1.532(7)$ & $\mathrm{C}(731)-\mathrm{C}(732)$ & $1.516(9)$ \\
\hline $\mathrm{C}(131)-\mathrm{C}(133)$ & $1.535(7)$ & $\mathrm{C}(731)-\mathrm{C}(734)$ & $1.523(8)$ \\
\hline $\mathrm{C}(151)-\mathrm{C}(153)$ & $1.518(7)$ & $\mathrm{C}(731)-\mathrm{C}(733)$ & $1.528(8)$ \\
\hline $\mathrm{C}(151)-\mathrm{C}(152)$ & $1.528(7)$ & $\mathrm{C}(751)-\mathrm{C}(753)$ & $1.524(8)$ \\
\hline $\mathrm{C}(151)-\mathrm{C}(154)$ & $1.539(7)$ & $\mathrm{C}(751)-\mathrm{C}(752)$ & $1.525(7)$ \\
\hline$C(231)-C(233)$ & $1.530(7)$ & $\mathrm{C}(751)-\mathrm{C}(754)$ & $1.531(8)$ \\
\hline
\end{tabular}




\begin{tabular}{|c|c|c|c|}
\hline $\mathrm{O}(1)-\mathrm{Ca}(1)-\mathrm{O}(1) \# 1$ & $79.84(15)$ & $\mathrm{N}(11) \# 1-\mathrm{Ca}(2)-\mathrm{C}(25)$ & $105.05(13)$ \\
\hline $\mathrm{O}(1)-\mathrm{Ca}(1)-\mathrm{O}(40)$ & $104.97(13)$ & $\mathrm{N}(21)-\mathrm{Ca}(2)-\mathrm{C}(25)$ & $25.74(11)$ \\
\hline $\mathrm{O}(1) \# 1-\mathrm{Ca}(1)-\mathrm{O}(40)$ & $164.87(12)$ & $\mathrm{N}(22)-\mathrm{Ca}(2)-\mathrm{C}(25)$ & $45.41(12)$ \\
\hline $\mathrm{O}(1)-\mathrm{Ca}(1)-\mathrm{N}(22)$ & $130.21(13)$ & $\mathrm{N}(31)-\mathrm{Ca}(2)-\mathrm{N}(12) \# 1$ & $126.48(12)$ \\
\hline $\mathrm{O}(1) \# 1-\mathrm{Ca}(1)-\mathrm{N}(22)$ & $81.84(12)$ & $\mathrm{O}(1) \# 1-\mathrm{Ca}(2)-\mathrm{N}(12) \# 1$ & $74.29(11)$ \\
\hline $\mathrm{O}(40)-\mathrm{Ca}(1)-\mathrm{N}(22)$ & $84.27(12)$ & $\mathrm{N}(32)-\mathrm{Ca}(2)-\mathrm{N}(12) \# 1$ & $136.05(12)$ \\
\hline $\mathrm{O}(1)-\mathrm{Ca}(1)-\mathrm{N}(12)$ & $91.31(13)$ & $\mathrm{N}(11) \# 1-\mathrm{Ca}(2)-\mathrm{N}(12) \# 1$ & $24.74(11)$ \\
\hline $\mathrm{O}(1) \# 1-\mathrm{Ca}(1)-\mathrm{N}(12)$ & $107.12(13)$ & $\mathrm{N}(21)-\mathrm{Ca}(2)-\mathrm{N}(12) \# 1$ & $88.90(11)$ \\
\hline $\mathrm{O}(40)-\mathrm{Ca}(1)-\mathrm{N}(12)$ & $87.29(12)$ & $\mathrm{N}(22)-\mathrm{Ca}(2)-\mathrm{N}(12) \# 1$ & $118.00(11)$ \\
\hline $\mathrm{N}(22)-\mathrm{Ca}(1)-\mathrm{N}(12)$ & $138.40(14)$ & $\mathrm{C}(25)-\mathrm{Ca}(2)-\mathrm{N}(12) \# 1$ & $91.30(11)$ \\
\hline $\mathrm{O}(1)-\mathrm{Ca}(1)-\mathrm{N}(21)$ & $97.58(13)$ & $\mathrm{N}(31)-\mathrm{Ca}(2)-\mathrm{C}(23)$ & $102.26(13)$ \\
\hline $\mathrm{O}(1) \# 1-\mathrm{Ca}(1)-\mathrm{N}(21)$ & $80.86(12)$ & $\mathrm{O}(1) \# 1-\mathrm{Ca}(2)-\mathrm{C}(23)$ & $101.54(12)$ \\
\hline $\mathrm{O}(40)-\mathrm{Ca}(1)-\mathrm{N}(21)$ & $84.27(12)$ & $\mathrm{N}(32)-\mathrm{Ca}(2)-\mathrm{C}(23)$ & $85.25(13)$ \\
\hline $\mathrm{N}(22)-\mathrm{Ca}(1)-\mathrm{N}(21)$ & $33.64(12)$ & $\mathrm{N}(11) \# 1-\mathrm{Ca}(2)-\mathrm{C}(23)$ & $146.43(13)$ \\
\hline $\mathrm{N}(12)-\mathrm{Ca}(1)-\mathrm{N}(21)$ & $169.06(13)$ & $\mathrm{N}(21)-\mathrm{Ca}(2)-\mathrm{C}(23)$ & $43.71(12)$ \\
\hline $\mathrm{Ca}(1)-\mathrm{O}(1)-\mathrm{Ca}(2) \# 1$ & $110.46(15)$ & $\mathrm{N}(22)-\mathrm{Ca}(2)-\mathrm{C}(23)$ & $24.30(11)$ \\
\hline $\mathrm{Ca}(1)-\mathrm{O}(1)-\mathrm{Ca}(1) \# 1$ & $100.16(15)$ & $\mathrm{C}(25)-\mathrm{Ca}(2)-\mathrm{C}(23)$ & $41.69(12)$ \\
\hline $\mathrm{Ca}(2) \# 1-\mathrm{O}(1)-\mathrm{Ca}(1) \# 1$ & $102.50(14)$ & $\mathrm{N}(12) \# 1-\mathrm{Ca}(2)-\mathrm{C}(23)$ & $131.19(11)$ \\
\hline $\mathrm{N}(31)-\mathrm{Ca}(2)-\mathrm{O}(1) \# 1$ & $94.98(14)$ & $\mathrm{Ca}(3)-\mathrm{O}(2)-\mathrm{Ca}(4)$ & $112.00(15)$ \\
\hline $\mathrm{N}(31)-\mathrm{Ca}(2)-\mathrm{N}(32)$ & $34.83(13)$ & $\mathrm{Ca}(3)-\mathrm{O}(2)-\mathrm{Ca}(3) \# 2$ & $99.99(14)$ \\
\hline $\mathrm{O}(1) \# 1-\mathrm{Ca}(2)-\mathrm{N}(32)$ & $128.48(14)$ & $\mathrm{Ca}(4)-\mathrm{O}(2)-\mathrm{Ca}(3) \# 2$ & $102.29(15)$ \\
\hline $\mathrm{N}(31)-\mathrm{Ca}(2)-\mathrm{N}(11) \# 1$ & $107.70(14)$ & $\mathrm{O}(2)-\mathrm{Ca}(3)-\mathrm{O}(2) \# 2$ & $80.01(14)$ \\
\hline $\mathrm{O}(1) \# 1-\mathrm{Ca}(2)-\mathrm{N}(11) \# 1$ & $90.75(13)$ & $\mathrm{O}(2)-\mathrm{Ca}(3)-\mathrm{O}(80)$ & $101.41(14)$ \\
\hline $\mathrm{N}(32)-\mathrm{Ca}(2)-\mathrm{N}(11) \# 1$ & 111.31(13) & $\mathrm{O}(2) \# 2-\mathrm{Ca}(3)-\mathrm{O}(80)$ & $168.71(14)$ \\
\hline $\mathrm{N}(31)-\mathrm{Ca}(2)-\mathrm{N}(21)$ & $141.66(14)$ & $\mathrm{O}(2)-\mathrm{Ca}(3)-\mathrm{N}(61)$ & $91.16(13)$ \\
\hline $\mathrm{O}(1) \# 1-\mathrm{Ca}(2)-\mathrm{N}(21)$ & 79.64(13) & $\mathrm{O}(2) \# 2-\mathrm{Ca}(3)-\mathrm{N}(61)$ & $107.06(14)$ \\
\hline $\mathrm{N}(32)-\mathrm{Ca}(2)-\mathrm{N}(21)$ & $128.23(13)$ & $\mathrm{O}(80)-\mathrm{Ca}(3)-\mathrm{N}(61)$ & $84.17(13)$ \\
\hline $\mathrm{N}(11) \# 1-\mathrm{Ca}(2)-\mathrm{N}(21)$ & $110.28(13)$ & $\mathrm{O}(2)-\mathrm{Ca}(3)-\mathrm{N}(52)$ & $129.03(14)$ \\
\hline $\mathrm{N}(31)-\mathrm{Ca}(2)-\mathrm{N}(22)$ & $110.07(13)$ & $\mathrm{O}(2) \# 2-\mathrm{Ca}(3)-\mathrm{N}(52)$ & $82.47(14)$ \\
\hline $\mathrm{O}(1) \# 1-\mathrm{Ca}(2)-\mathrm{N}(22)$ & $77.95(12)$ & $\mathrm{O}(80)-\mathrm{Ca}(3)-\mathrm{N}(52)$ & $88.11(14)$ \\
\hline $\mathrm{N}(32)-\mathrm{Ca}(2)-\mathrm{N}(22)$ & $104.45(13)$ & $\mathrm{N}(61)-\mathrm{Ca}(3)-\mathrm{N}(52)$ & $139.79(14)$ \\
\hline $\mathrm{N}(11) \# 1-\mathrm{Ca}(2)-\mathrm{N}(22)$ & $141.29(13)$ & $\mathrm{O}(2)-\mathrm{Ca}(3)-\mathrm{N}(51)$ & $96.83(14)$ \\
\hline $\mathrm{N}(21)-\mathrm{Ca}(2)-\mathrm{N}(22)$ & $31.60(12)$ & $\mathrm{O}(2) \# 2-\mathrm{Ca}(3)-\mathrm{N}(51)$ & $81.64(14)$ \\
\hline $\mathrm{N}(31)-\mathrm{Ca}(2)-\mathrm{C}(25)$ & $141.16(14)$ & $\mathrm{O}(80)-\mathrm{Ca}(3)-\mathrm{N}(51)$ & $87.07(13)$ \\
\hline $\mathrm{O}(1) \# 1-\mathrm{Ca}(2)-\mathrm{C}(25)$ & $104.96(13)$ & $\mathrm{N}(61)-\mathrm{Ca}(3)-\mathrm{N}(51)$ & $169.16(14)$ \\
\hline $\mathrm{N}(32)-\mathrm{Ca}(2)-\mathrm{C}(25)$ & $112.47(13)$ & $\mathrm{N}(52)-\mathrm{Ca}(3)-\mathrm{N}(51)$ & $33.12(13)$ \\
\hline
\end{tabular}




\begin{tabular}{|c|c|c|c|}
\hline $\mathrm{N}(72)-\mathrm{Ca}(4)-\mathrm{O}(2)$ & $91.20(14)$ & $\mathrm{Ca}(1)-\mathrm{N}(21)-\mathrm{Ca}(2)$ & $93.82(13)$ \\
\hline $\mathrm{N}(72)-\mathrm{Ca}(4)-\mathrm{N}(71)$ & $34.38(13)$ & $\mathrm{C}(23)-\mathrm{N}(22)-\mathrm{N}(21)$ & $107.4(4)$ \\
\hline $\mathrm{O}(2)-\mathrm{Ca}(4)-\mathrm{N}(71)$ & $125.27(14)$ & $\mathrm{C}(23)-\mathrm{N}(22)-\mathrm{Ca}(1)$ & $165.5(3)$ \\
\hline $\mathrm{N}(72)-\mathrm{Ca}(4)-\mathrm{N}(62)$ & $114.19(15)$ & $\mathrm{N}(21)-\mathrm{N}(22)-\mathrm{Ca}(1)$ & $74.3(2)$ \\
\hline $\mathrm{O}(2)-\mathrm{Ca}(4)-\mathrm{N}(62)$ & $93.08(13)$ & $\mathrm{C}(23)-\mathrm{N}(22)-\mathrm{Ca}(2)$ & $102.2(3)$ \\
\hline $\mathrm{N}(71)-\mathrm{Ca}(4)-\mathrm{N}(62)$ & $111.59(14)$ & $\mathrm{N}(21)-\mathrm{N}(22)-\mathrm{Ca}(2)$ & $70.3(2)$ \\
\hline $\mathrm{N}(72)-\mathrm{Ca}(4)-\mathrm{N}(51) \# 2$ & $142.16(15)$ & $\mathrm{Ca}(1)-\mathrm{N}(22)-\mathrm{Ca}(2)$ & $92.02(12)$ \\
\hline $\mathrm{O}(2)-\mathrm{Ca}(4)-\mathrm{N}(51) \# 2$ & $80.19(14)$ & $\mathrm{N}(22)-\mathrm{C}(23)-\mathrm{C}(24)$ & $109.4(4)$ \\
\hline $\mathrm{N}(71)-\mathrm{Ca}(4)-\mathrm{N}(51) \# 2$ & $134.09(15)$ & $\mathrm{N}(22)-\mathrm{C}(23)-\mathrm{C}(231)$ & $119.5(4)$ \\
\hline $\mathrm{N}(62)-\mathrm{Ca}(4)-\mathrm{N}(51) \# 2$ & $103.09(14)$ & $C(24)-C(23)-C(231)$ & $130.9(4)$ \\
\hline $\mathrm{N}(72)-\mathrm{Ca}(4)-\mathrm{N}(52) \# 2$ & $110.63(15)$ & $\mathrm{N}(22)-\mathrm{C}(23)-\mathrm{Ca}(2)$ & $53.5(2)$ \\
\hline $\mathrm{O}(2)-\mathrm{Ca}(4)-\mathrm{N}(52) \# 2$ & $79.95(13)$ & $\mathrm{C}(24)-\mathrm{C}(23)-\mathrm{Ca}(2)$ & $88.0(3)$ \\
\hline $\mathrm{N}(71)-\mathrm{Ca}(4)-\mathrm{N}(52) \# 2$ & $108.60(14)$ & $\mathrm{C}(231)-\mathrm{C}(23)-\mathrm{Ca}(2)$ & $123.8(3)$ \\
\hline $\mathrm{N}(62)-\mathrm{Ca}(4)-\mathrm{N}(52) \# 2$ & $134.76(14)$ & $\mathrm{C}(25)-\mathrm{C}(24)-\mathrm{C}(23)$ & $106.4(4)$ \\
\hline $\mathrm{N}(51) \# 2-\mathrm{Ca}(4)-\mathrm{N}(52) \# 2$ & $31.71(13)$ & $\mathrm{N}(21)-\mathrm{C}(25)-\mathrm{C}(24)$ & $108.6(4)$ \\
\hline $\mathrm{C}(15)-\mathrm{N}(11)-\mathrm{N}(12)$ & $107.4(4)$ & $\mathrm{N}(21)-\mathrm{C}(25)-\mathrm{C}(251)$ & $120.8(4)$ \\
\hline $\mathrm{C}(15)-\mathrm{N}(11)-\mathrm{Ca}(2) \# 1$ & $137.3(3)$ & $C(24)-C(25)-C(251)$ & $130.6(4)$ \\
\hline $\mathrm{N}(12)-\mathrm{N}(11)-\mathrm{Ca}(2) \# 1$ & $109.0(3)$ & $\mathrm{N}(21)-\mathrm{C}(25)-\mathrm{Ca}(2)$ & $54.3(2)$ \\
\hline $\mathrm{C}(13)-\mathrm{N}(12)-\mathrm{N}(11)$ & $107.9(4)$ & $\mathrm{C}(24)-\mathrm{C}(25)-\mathrm{Ca}(2)$ & $93.4(3)$ \\
\hline $\mathrm{C}(13)-\mathrm{N}(12)-\mathrm{Ca}(1)$ & $128.3(3)$ & $\mathrm{C}(251)-\mathrm{C}(25)-\mathrm{Ca}(2)$ & $113.8(3)$ \\
\hline $\mathrm{N}(11)-\mathrm{N}(12)-\mathrm{Ca}(1)$ & $112.7(3)$ & $\mathrm{C}(35)-\mathrm{N}(31)-\mathrm{N}(32)$ & $108.3(4)$ \\
\hline $\mathrm{C}(13)-\mathrm{N}(12)-\mathrm{Ca}(2) \# 1$ & $147.6(3)$ & $\mathrm{C}(35)-\mathrm{N}(31)-\mathrm{Ca}(2)$ & $175.7(3)$ \\
\hline $\mathrm{N}(11)-\mathrm{N}(12)-\mathrm{Ca}(2) \# 1$ & $46.2(2)$ & $\mathrm{N}(32)-\mathrm{N}(31)-\mathrm{Ca}(2)$ & $75.9(2)$ \\
\hline $\mathrm{Ca}(1)-\mathrm{N}(12)-\mathrm{Ca}(2) \# 1$ & $83.94(11)$ & $\mathrm{C}(33)-\mathrm{N}(32)-\mathrm{N}(31)$ & $106.7(4)$ \\
\hline $\mathrm{N}(12)-\mathrm{C}(13)-\mathrm{C}(14)$ & $109.3(4)$ & $\mathrm{C}(33)-\mathrm{N}(32)-\mathrm{Ca}(2)$ & $176.0(3)$ \\
\hline $\mathrm{N}(12)-\mathrm{C}(13)-\mathrm{C}(131)$ & $122.8(4)$ & $\mathrm{N}(31)-\mathrm{N}(32)-\mathrm{Ca}(2)$ & $69.2(2)$ \\
\hline$C(14)-C(13)-C(131)$ & $127.9(4)$ & $\mathrm{N}(32)-\mathrm{C}(33)-\mathrm{C}(34)$ & $110.0(4)$ \\
\hline $\mathrm{C}(15)-\mathrm{C}(14)-\mathrm{C}(13)$ & $106.3(4)$ & $\mathrm{N}(32)-\mathrm{C}(33)-\mathrm{C}(331)$ & $122.3(4)$ \\
\hline $\mathrm{N}(11)-\mathrm{C}(15)-\mathrm{C}(14)$ & $109.1(4)$ & $\mathrm{C}(34)-\mathrm{C}(33)-\mathrm{C}(331)$ & $127.7(4)$ \\
\hline $\mathrm{N}(11)-\mathrm{C}(15)-\mathrm{C}(151)$ & $121.6(4)$ & $\mathrm{C}(35)-\mathrm{C}(34)-\mathrm{C}(33)$ & $105.4(4)$ \\
\hline$C(14)-C(15)-C(151)$ & $129.3(4)$ & $\mathrm{N}(31)-\mathrm{C}(35)-\mathrm{C}(34)$ & $109.6(4)$ \\
\hline $\mathrm{C}(25)-\mathrm{N}(21)-\mathrm{N}(22)$ & $108.2(4)$ & $\mathrm{N}(31)-\mathrm{C}(35)-\mathrm{C}(351)$ & $118.9(4)$ \\
\hline $\mathrm{C}(25)-\mathrm{N}(21)-\mathrm{Ca}(1)$ & $166.0(3)$ & $\mathrm{C}(34)-\mathrm{C}(35)-\mathrm{C}(351)$ & $131.6(5)$ \\
\hline $\mathrm{N}(22)-\mathrm{N}(21)-\mathrm{Ca}(1)$ & $72.1(2)$ & $\mathrm{C}(44)-\mathrm{O}(40)-\mathrm{C}(41)$ & $106.4(4)$ \\
\hline $\mathrm{C}(25)-\mathrm{N}(21)-\mathrm{Ca}(2)$ & $99.9(3)$ & $\mathrm{C}(44)-\mathrm{O}(40)-\mathrm{Ca}(1)$ & $126.3(3)$ \\
\hline $\mathrm{N}(22)-\mathrm{N}(21)-\mathrm{Ca}(2)$ & $78.1(2)$ & $\mathrm{C}(41)-\mathrm{O}(40)-\mathrm{Ca}(1)$ & $126.9(3)$ \\
\hline
\end{tabular}




\begin{tabular}{|c|c|c|c|}
\hline $\mathrm{O}(40)-\mathrm{C}(41)-\mathrm{C}(42)$ & $105.6(5)$ & $\mathrm{C}(75)-\mathrm{N}(71)-\mathrm{N}(72)$ & $107.3(4)$ \\
\hline $\mathrm{C}(41)-\mathrm{C}(42)-\mathrm{C}(43)$ & $105.8(5)$ & $\mathrm{C}(75)-\mathrm{N}(71)-\mathrm{Ca}(4)$ & 175.1(3) \\
\hline $\mathrm{C}(42)-\mathrm{C}(43)-\mathrm{C}(44)$ & $104.2(4)$ & $\mathrm{N}(72)-\mathrm{N}(71)-\mathrm{Ca}(4)$ & $70.2(2)$ \\
\hline $\mathrm{O}(40)-\mathrm{C}(44)-\mathrm{C}(43)$ & $106.5(4)$ & $\mathrm{C}(73)-\mathrm{N}(72)-\mathrm{N}(71)$ & $108.3(4)$ \\
\hline $\mathrm{C}(55)-\mathrm{N}(51)-\mathrm{N}(52)$ & $108.4(4)$ & $\mathrm{C}(73)-\mathrm{N}(72)-\mathrm{Ca}(4)$ & $174.4(3)$ \\
\hline $\mathrm{C}(55)-\mathrm{N}(51)-\mathrm{Ca}(3)$ & $159.0(4)$ & $\mathrm{N}(71)-\mathrm{N}(72)-\mathrm{Ca}(4)$ & $75.4(2)$ \\
\hline $\mathrm{N}(52)-\mathrm{N}(51)-\mathrm{Ca}(3)$ & $72.8(2)$ & $\mathrm{N}(72)-\mathrm{C}(73)-\mathrm{C}(74)$ & $109.3(5)$ \\
\hline $\mathrm{C}(55)-\mathrm{N}(51)-\mathrm{Ca}(4) \# 2$ & 109.1(3) & $\mathrm{N}(72)-\mathrm{C}(73)-\mathrm{C}(731)$ & $120.2(5)$ \\
\hline $\mathrm{N}(52)-\mathrm{N}(51)-\mathrm{Ca}(4) \# 2$ & $75.4(2)$ & $C(74)-C(73)-C(731)$ & $130.4(5)$ \\
\hline $\mathrm{Ca}(3)-\mathrm{N}(51)-\mathrm{Ca}(4) \# 2$ & $91.57(14)$ & $C(75)-C(74)-C(73)$ & $105.4(5)$ \\
\hline $\mathrm{C}(53)-\mathrm{N}(52)-\mathrm{N}(51)$ & $106.9(4)$ & $\mathrm{N}(71)-\mathrm{C}(75)-\mathrm{C}(74)$ & $109.6(4)$ \\
\hline $\mathrm{C}(53)-\mathrm{N}(52)-\mathrm{Ca}(3)$ & $159.5(4)$ & $\mathrm{N}(71)-\mathrm{C}(75)-\mathrm{C}(751)$ & $122.5(5)$ \\
\hline $\mathrm{N}(51)-\mathrm{N}(52)-\mathrm{Ca}(3)$ & $74.1(2)$ & $C(74)-C(75)-C(751)$ & $127.8(5)$ \\
\hline $\mathrm{C}(53)-\mathrm{N}(52)-\mathrm{Ca}(4) \# 2$ & $108.9(3)$ & $\mathrm{C}(84)-\mathrm{O}(80)-\mathrm{C}(81)$ & $109.0(4)$ \\
\hline $\mathrm{N}(51)-\mathrm{N}(52)-\mathrm{Ca}(4) \# 2$ & $72.9(2)$ & $\mathrm{C}(84)-\mathrm{O}(80)-\mathrm{Ca}(3)$ & $126.9(3)$ \\
\hline $\mathrm{Ca}(3)-\mathrm{N}(52)-\mathrm{Ca}(4) \# 2$ & $91.16(14)$ & $\mathrm{C}(81)-\mathrm{O}(80)-\mathrm{Ca}(3)$ & $123.7(3)$ \\
\hline $\mathrm{N}(52)-\mathrm{C}(53)-\mathrm{C}(54)$ & $109.4(5)$ & $\mathrm{O}(80)-\mathrm{C}(81)-\mathrm{C}(82)$ & $105.4(6)$ \\
\hline $\mathrm{N}(52)-\mathrm{C}(53)-\mathrm{C}(531)$ & $120.0(5)$ & $\mathrm{C}(81)-\mathrm{C}(82)-\mathrm{C}(83)$ & $103.6(8)$ \\
\hline$C(54)-C(53)-C(531)$ & $130.5(6)$ & $\mathrm{C}(81)-\mathrm{C}(82)-\mathrm{C}(85)$ & $105.9(8)$ \\
\hline$C(53)-C(54)-C(55)$ & $107.3(5)$ & $\mathrm{C}(83)-\mathrm{C}(82)-\mathrm{C}(85)$ & $35.6(7)$ \\
\hline $\mathrm{N}(51)-\mathrm{C}(55)-\mathrm{C}(54)$ & $108.0(5)$ & $\mathrm{C}(84)-\mathrm{C}(83)-\mathrm{C}(82)$ & $103.4(12)$ \\
\hline $\mathrm{N}(51)-\mathrm{C}(55)-\mathrm{C}(551)$ & $122.6(5)$ & $\mathrm{C}(83)-\mathrm{C}(84)-\mathrm{O}(80)$ & 107.2(8) \\
\hline $\mathrm{C}(54)-\mathrm{C}(55)-\mathrm{C}(551)$ & $129.4(5)$ & $\mathrm{C}(83)-\mathrm{C}(84)-\mathrm{C}(85)$ & $38.6(8)$ \\
\hline $\mathrm{C}(65)-\mathrm{N}(61)-\mathrm{N}(62)$ & $108.0(4)$ & $\mathrm{O}(80)-\mathrm{C}(84)-\mathrm{C}(85)$ & $110.5(8)$ \\
\hline $\mathrm{C}(65)-\mathrm{N}(61)-\mathrm{Ca}(3)$ & 127.1(3) & $\mathrm{C}(84)-\mathrm{C}(85)-\mathrm{C}(82)$ & $100.5(11)$ \\
\hline $\mathrm{N}(62)-\mathrm{N}(61)-\mathrm{Ca}(3)$ & $118.1(3)$ & $C(13)-C(131)-C(132)$ & $112.8(4)$ \\
\hline $\mathrm{C}(63)-\mathrm{N}(62)-\mathrm{N}(61)$ & $107.0(4)$ & $C(13)-C(131)-C(134)$ & $108.4(4)$ \\
\hline $\mathrm{C}(63)-\mathrm{N}(62)-\mathrm{Ca}(4)$ & $138.1(3)$ & $\mathrm{C}(132)-\mathrm{C}(131)-\mathrm{C}(134)$ & $108.9(4)$ \\
\hline $\mathrm{N}(61)-\mathrm{N}(62)-\mathrm{Ca}(4)$ & $112.8(3)$ & $C(13)-C(131)-C(133)$ & $109.6(4)$ \\
\hline $\mathrm{N}(62)-\mathrm{C}(63)-\mathrm{C}(64)$ & $109.5(5)$ & $\mathrm{C}(132)-\mathrm{C}(131)-\mathrm{C}(133)$ & $108.7(4)$ \\
\hline $\mathrm{N}(62)-\mathrm{C}(63)-\mathrm{C}(631)$ & $122.1(5)$ & $\mathrm{C}(134)-\mathrm{C}(131)-\mathrm{C}(133)$ & $108.4(4)$ \\
\hline $\mathrm{C}(64)-\mathrm{C}(63)-\mathrm{C}(631)$ & $128.3(5)$ & $C(15)-C(151)-C(153)$ & $111.5(4)$ \\
\hline$C(63)-C(64)-C(65)$ & $105.5(4)$ & $C(15)-C(151)-C(152)$ & 109.6(4) \\
\hline $\mathrm{N}(61)-\mathrm{C}(65)-\mathrm{C}(64)$ & $110.1(4)$ & $\mathrm{C}(153)-\mathrm{C}(151)-\mathrm{C}(152)$ & $109.0(4)$ \\
\hline $\mathrm{N}(61)-\mathrm{C}(65)-\mathrm{C}(651)$ & $123.7(4)$ & $C(15)-C(151)-C(154)$ & $109.0(4)$ \\
\hline$C(64)-C(65)-C(651)$ & $126.1(5)$ & $C(153)-C(151)-C(154)$ & $109.6(4)$ \\
\hline
\end{tabular}




\begin{tabular}{|c|c|c|c|}
\hline $\mathrm{C}(152)-\mathrm{C}(151)-\mathrm{C}(154)$ & $108.1(4)$ & $\mathrm{C}(55)-\mathrm{C}(551)-\mathrm{C}(552)$ & $110.4(5)$ \\
\hline$C(23)-C(231)-C(233)$ & $110.4(4)$ & $C(55)-C(551)-C(553)$ & $110.5(5)$ \\
\hline$C(23)-C(231)-C(232)$ & $109.8(4)$ & $C(552)-C(551)-C(553)$ & $111.5(6)$ \\
\hline $\mathrm{C}(233)-\mathrm{C}(231)-\mathrm{C}(232)$ & $109.3(4)$ & $\mathrm{C}(55)-\mathrm{C}(551)-\mathrm{C}(554)$ & $110.2(5)$ \\
\hline$C(23)-C(231)-C(234)$ & $108.6(4)$ & $\mathrm{C}(552)-\mathrm{C}(551)-\mathrm{C}(554)$ & $107.5(7)$ \\
\hline $\mathrm{C}(233)-\mathrm{C}(231)-\mathrm{C}(234)$ & $109.7(4)$ & $C(553)-C(551)-C(554)$ & $106.7(6)$ \\
\hline $\mathrm{C}(232)-\mathrm{C}(231)-\mathrm{C}(234)$ & $108.9(4)$ & $\mathrm{C}(63)-\mathrm{C}(631)-\mathrm{C}(634)$ & $110.1(5)$ \\
\hline$C(25)-C(251)-C(52)$ & $109.9(4)$ & $C(63)-C(631)-C(632)$ & $109.7(5)$ \\
\hline$C(25)-C(251)-C(254)$ & $110.1(4)$ & $\mathrm{C}(634)-\mathrm{C}(631)-\mathrm{C}(632)$ & $108.9(5)$ \\
\hline$C(52)-C(251)-C(254)$ & 109.3(4) & $\mathrm{C}(63)-\mathrm{C}(631)-\mathrm{C}(633)$ & $110.4(5)$ \\
\hline$C(25)-C(251)-C(253)$ & 109.9(4) & $\mathrm{C}(634)-\mathrm{C}(631)-\mathrm{C}(633)$ & $110.0(5)$ \\
\hline$C(52)-C(251)-C(253)$ & 109.2(4) & $\mathrm{C}(632)-\mathrm{C}(631)-\mathrm{C}(633)$ & $107.8(5)$ \\
\hline $\mathrm{C}(254)-\mathrm{C}(251)-\mathrm{C}(253)$ & $108.5(4)$ & $\mathrm{C}(65)-\mathrm{C}(651)-\mathrm{C}(652)$ & $111.6(4)$ \\
\hline$C(33)-C(331)-C(334)$ & $111.5(4)$ & $\mathrm{C}(65)-\mathrm{C}(651)-\mathrm{C}(653)$ & $110.6(5)$ \\
\hline $\mathrm{C}(33)-\mathrm{C}(331)-\mathrm{C}(333)$ & 109.3(4) & $\mathrm{C}(652)-\mathrm{C}(651)-\mathrm{C}(653)$ & $108.6(5)$ \\
\hline $\mathrm{C}(334)-\mathrm{C}(331)-\mathrm{C}(333)$ & 109.2(4) & $\mathrm{C}(65)-\mathrm{C}(651)-\mathrm{C}(654)$ & $107.9(5)$ \\
\hline $\mathrm{C}(33)-\mathrm{C}(331)-\mathrm{C}(332)$ & $109.5(4)$ & $\mathrm{C}(652)-\mathrm{C}(651)-\mathrm{C}(654)$ & $109.5(5)$ \\
\hline $\mathrm{C}(334)-\mathrm{C}(331)-\mathrm{C}(332)$ & $108.3(4)$ & $\mathrm{C}(653)-\mathrm{C}(651)-\mathrm{C}(654)$ & $108.6(5)$ \\
\hline $\mathrm{C}(333)-\mathrm{C}(331)-\mathrm{C}(332)$ & $108.9(4)$ & $\mathrm{C}(73)-\mathrm{C}(731)-\mathrm{C}(732)$ & $110.5(5)$ \\
\hline $\mathrm{C}(352)-\mathrm{C}(351)-\mathrm{C}(354)$ & $114.1(6)$ & $\mathrm{C}(73)-\mathrm{C}(731)-\mathrm{C}(734)$ & $110.7(5)$ \\
\hline $\mathrm{C}(352)-\mathrm{C}(351)-\mathrm{C}(35)$ & $108.8(4)$ & $\mathrm{C}(732)-\mathrm{C}(731)-\mathrm{C}(734)$ & $109.0(6)$ \\
\hline$C(354)-C(351)-C(35)$ & $110.8(4)$ & $\mathrm{C}(73)-\mathrm{C}(731)-\mathrm{C}(733)$ & $107.9(5)$ \\
\hline $\mathrm{C}(352)-\mathrm{C}(351)-\mathrm{C}(353)$ & $108.0(6)$ & $\mathrm{C}(732)-\mathrm{C}(731)-\mathrm{C}(733)$ & $108.9(5)$ \\
\hline $\mathrm{C}(354)-\mathrm{C}(351)-\mathrm{C}(353)$ & $106.9(5)$ & $\mathrm{C}(734)-\mathrm{C}(731)-\mathrm{C}(733)$ & $109.7(5)$ \\
\hline $\mathrm{C}(35)-\mathrm{C}(351)-\mathrm{C}(353)$ & $108.0(4)$ & $\mathrm{C}(75)-\mathrm{C}(751)-\mathrm{C}(753)$ & $111.9(4)$ \\
\hline $\mathrm{C}(533)-\mathrm{C}(531)-\mathrm{C}(534)$ & $111.6(6)$ & $C(75)-C(751)-C(752)$ & $109.8(4)$ \\
\hline $\mathrm{C}(533)-\mathrm{C}(531)-\mathrm{C}(53)$ & $109.7(5)$ & $\mathrm{C}(753)-\mathrm{C}(751)-\mathrm{C}(752)$ & $109.4(5)$ \\
\hline $\mathrm{C}(534)-\mathrm{C}(531)-\mathrm{C}(53)$ & $109.2(5)$ & $C(75)-C(751)-C(754)$ & $109.7(5)$ \\
\hline $\mathrm{C}(533)-\mathrm{C}(531)-\mathrm{C}(532)$ & $109.4(6)$ & $\mathrm{C}(753)-\mathrm{C}(751)-\mathrm{C}(754)$ & $108.5(5)$ \\
\hline $\mathrm{C}(534)-\mathrm{C}(531)-\mathrm{C}(532)$ & $109.2(5)$ & $\mathrm{C}(752)-\mathrm{C}(751)-\mathrm{C}(754)$ & $107.5(5)$ \\
\hline $\mathrm{C}(53)-\mathrm{C}(531)-\mathrm{C}(532)$ & $107.7(5)$ & & \\
\hline
\end{tabular}

Symmetry transformations used to generate equivalent atoms:

$\# 1-x+1,-y+1,-z+1 \quad \# 2-x,-y,-z+2$ 
Table 4. Anisotropic displacement parameters $\left(\AA^{2} \times 10^{3}\right)$ for compound 4. The anisotropic displacement factor exponent takes the form: $-2 \pi^{2}\left[h^{2} a^{* 2} U^{11}+\ldots+2 h k^{*} b^{*} U^{12}\right]$

\begin{tabular}{|c|c|c|c|c|c|c|}
\hline & $\mathrm{U}^{11}$ & $\mathrm{U}^{22}$ & $\mathrm{U}^{33}$ & $\mathrm{U}^{23}$ & $\mathrm{U}^{13}$ & $\mathrm{U}^{12}$ \\
\hline $\mathrm{Ca}(1)$ & $21(1)$ & $16(1)$ & $29(1)$ & $-6(1)$ & $-3(1)$ & $-8(1)$ \\
\hline $\mathrm{O}(1)$ & $25(2)$ & $17(2)$ & $21(2)$ & $-5(2)$ & $-2(2)$ & $-8(2)$ \\
\hline $\mathrm{Ca}(2)$ & $24(1)$ & $15(1)$ & $26(1)$ & $-2(1)$ & $-5(1)$ & $-6(1)$ \\
\hline $\mathrm{O}(2)$ & $25(2)$ & $24(2)$ & $24(2)$ & $-8(2)$ & $-5(2)$ & $-10(2)$ \\
\hline $\mathrm{Ca}(3)$ & $27(1)$ & $23(1)$ & $35(1)$ & $-11(1)$ & $-2(1)$ & $-11(1)$ \\
\hline $\mathrm{Ca}(4)$ & $27(1)$ & $32(1)$ & $30(1)$ & $-13(1)$ & $-2(1)$ & $-8(1)$ \\
\hline $\mathrm{N}(11)$ & $25(2)$ & $18(2)$ & $26(2)$ & $-3(2)$ & $-5(2)$ & $-8(2)$ \\
\hline $\mathrm{N}(12)$ & $20(2)$ & $19(2)$ & $29(2)$ & $-9(2)$ & $-1(2)$ & $-9(2)$ \\
\hline$C(13)$ & $26(3)$ & $20(3)$ & $34(3)$ & $-7(2)$ & $-4(2)$ & $-11(2)$ \\
\hline $\mathrm{C}(14)$ & $17(3)$ & $24(3)$ & $39(3)$ & $-7(2)$ & $-1(2)$ & $-8(2)$ \\
\hline$C(15)$ & $22(3)$ & $18(3)$ & $32(3)$ & $-10(2)$ & $-4(2)$ & $-8(2)$ \\
\hline $\mathrm{N}(21)$ & $25(2)$ & $17(2)$ & $28(2)$ & $-6(2)$ & $-3(2)$ & $-9(2)$ \\
\hline $\mathrm{N}(22)$ & $32(2)$ & $13(2)$ & $29(2)$ & $-4(2)$ & $-6(2)$ & $-10(2)$ \\
\hline $\mathrm{C}(23)$ & $20(3)$ & $17(3)$ & $31(3)$ & $-2(2)$ & $-10(2)$ & $-8(2)$ \\
\hline$C(24)$ & $26(3)$ & $14(2)$ & $36(3)$ & $-9(2)$ & $-4(2)$ & $-3(2)$ \\
\hline$C(25)$ & $17(2)$ & $16(2)$ & $31(3)$ & $-9(2)$ & $-5(2)$ & $-3(2)$ \\
\hline $\mathrm{N}(31)$ & $20(2)$ & $23(2)$ & $27(2)$ & $-7(2)$ & $-3(2)$ & $-6(2)$ \\
\hline $\mathrm{N}(32)$ & $20(2)$ & $18(2)$ & $29(2)$ & $-9(2)$ & $2(2)$ & $-5(2)$ \\
\hline$C(33)$ & $19(2)$ & $23(3)$ & $22(3)$ & $-2(2)$ & $-3(2)$ & $-6(2)$ \\
\hline $\mathrm{C}(34)$ & $29(3)$ & $27(3)$ & $21(3)$ & $3(2)$ & $-7(2)$ & $-11(2)$ \\
\hline$C(35)$ & $22(3)$ & $25(3)$ & $29(3)$ & $-4(2)$ & $-4(2)$ & $-10(2)$ \\
\hline $\mathrm{O}(40)$ & $30(2)$ & $22(2)$ & $55(2)$ & $-20(2)$ & $-16(2)$ & $-2(2)$ \\
\hline $\mathrm{C}(41)$ & $43(3)$ & $29(3)$ & $56(4)$ & $-20(3)$ & $-18(3)$ & $-8(3)$ \\
\hline $\mathrm{C}(42)$ & $74(5)$ & $41(4)$ & $62(5)$ & $-21(3)$ & $-30(4)$ & $6(3)$ \\
\hline $\mathrm{C}(43)$ & $45(3)$ & $40(3)$ & $45(4)$ & $-4(3)$ & $-16(3)$ & $-21(3)$ \\
\hline $\mathrm{C}(44)$ & $44(4)$ & $34(3)$ & $68(4)$ & $-20(3)$ & $-27(3)$ & $-4(3)$ \\
\hline $\mathrm{N}(51)$ & $34(3)$ & $31(3)$ & $35(3)$ & $-7(2)$ & $-8(2)$ & $-14(2)$ \\
\hline $\mathrm{N}(52)$ & $32(3)$ & $37(3)$ & $37(3)$ & $-6(2)$ & $0(2)$ & $-19(2)$ \\
\hline $\mathrm{C}(52)$ & $42(3)$ & $28(3)$ & $31(3)$ & $-4(2)$ & $-1(3)$ & $-9(3)$ \\
\hline $\mathrm{C}(53)$ & $45(4)$ & $43(3)$ & $42(4)$ & $-20(3)$ & $14(3)$ & $-27(3)$ \\
\hline $\mathrm{C}(54)$ & $87(5)$ & $73(5)$ & $24(3)$ & $-9(3)$ & $6(3)$ & $-54(4)$ \\
\hline
\end{tabular}




\begin{tabular}{|c|c|c|c|c|c|c|}
\hline$C(55)$ & $65(4)$ & $33(3)$ & 29(3) & $-4(3)$ & $-10(3)$ & $-28(3)$ \\
\hline $\mathrm{N}(61)$ & $24(2)$ & $26(2)$ & $34(3)$ & $-9(2)$ & $-9(2)$ & $-5(2)$ \\
\hline $\mathrm{N}(62)$ & $26(2)$ & $33(2)$ & $33(3)$ & $-15(2)$ & $-4(2)$ & $-7(2)$ \\
\hline$C(63)$ & $37(3)$ & $35(3)$ & $32(3)$ & $-10(3)$ & $-10(3)$ & $-14(3)$ \\
\hline$C(64)$ & $40(3)$ & $38(3)$ & $38(3)$ & $-15(3)$ & $-12(3)$ & $-17(3)$ \\
\hline$C(65)$ & $28(3)$ & $30(3)$ & $30(3)$ & $-8(2)$ & $-8(2)$ & $-11(2)$ \\
\hline $\mathrm{N}(71)$ & $26(2)$ & $34(2)$ & $26(2)$ & $-11(2)$ & $-1(2)$ & $-13(2)$ \\
\hline $\mathrm{N}(72)$ & $33(3)$ & $34(3)$ & $28(3)$ & $-11(2)$ & $-4(2)$ & $-12(2)$ \\
\hline$C(73)$ & $36(3)$ & $30(3)$ & $37(3)$ & $-15(3)$ & $-8(3)$ & $-5(3)$ \\
\hline$C(74)$ & $27(3)$ & $28(3)$ & $44(4)$ & $-16(3)$ & $-8(3)$ & $2(2)$ \\
\hline$C(75)$ & $30(3)$ & $24(3)$ & $34(3)$ & $-15(2)$ & $8(2)$ & $-11(2)$ \\
\hline $\mathrm{O}(80)$ & $72(3)$ & $26(2)$ & $35(2)$ & $-3(2)$ & $-9(2)$ & $-16(2)$ \\
\hline $\mathrm{C}(81)$ & $109(6)$ & $28(4)$ & $57(5)$ & $-14(3)$ & $-7(4)$ & $-11(4)$ \\
\hline $\mathrm{C}(82)$ & $169(10)$ & $42(5)$ & $87(6)$ & $-8(4)$ & $-15(7)$ & $-25(5)$ \\
\hline $\mathrm{C}(84)$ & $110(6)$ & $31(4)$ & $55(5)$ & $-2(3)$ & $4(4)$ & $-21(4)$ \\
\hline $\mathrm{C}(131)$ & $29(3)$ & $24(3)$ & $38(3)$ & $-8(2)$ & $-2(2)$ & $-13(2)$ \\
\hline$C(132)$ & $32(3)$ & $33(3)$ & $42(3)$ & $3(3)$ & $-7(3)$ & $-13(3)$ \\
\hline$C(133)$ & $42(3)$ & $30(3)$ & $64(4)$ & $5(3)$ & $-12(3)$ & $-21(3)$ \\
\hline$C(134)$ & $57(4)$ & $38(3)$ & $34(3)$ & $-5(3)$ & $0(3)$ & $-14(3)$ \\
\hline$C(151)$ & 23(3) & $21(3)$ & $38(3)$ & $-4(2)$ & $-10(2)$ & $-7(2)$ \\
\hline$C(152)$ & $34(3)$ & $22(3)$ & 44(3) & $-5(3)$ & $-6(3)$ & $-6(2)$ \\
\hline$C(153)$ & $36(3)$ & $33(3)$ & $36(3)$ & $-4(3)$ & $-10(3)$ & $-10(3)$ \\
\hline$C(154)$ & 27(3) & $42(3)$ & $53(4)$ & $-5(3)$ & $-13(3)$ & $-11(3)$ \\
\hline$C(231)$ & $35(3)$ & $16(3)$ & $33(3)$ & $0(2)$ & $-3(2)$ & $-16(2)$ \\
\hline$C(232)$ & $42(3)$ & $19(3)$ & $46(4)$ & $0(2)$ & $-11(3)$ & $-18(2)$ \\
\hline$C(233)$ & $40(3)$ & $28(3)$ & $35(3)$ & $-1(2)$ & $-5(3)$ & $-20(3)$ \\
\hline$C(234)$ & $33(3)$ & $30(3)$ & $47(4)$ & $-10(3)$ & $-4(3)$ & $-15(3)$ \\
\hline$C(251)$ & $26(3)$ & $16(2)$ & $32(3)$ & $-11(2)$ & $2(2)$ & $-6(2)$ \\
\hline$C(253)$ & $47(3)$ & $38(3)$ & $35(3)$ & $-19(3)$ & $7(3)$ & $-23(3)$ \\
\hline$C(254)$ & $30(3)$ & $31(3)$ & 49(4) & $-20(3)$ & $-2(3)$ & $-8(2)$ \\
\hline$C(331)$ & $29(3)$ & $19(3)$ & $34(3)$ & $2(2)$ & $-6(2)$ & $-12(2)$ \\
\hline$C(332)$ & $52(4)$ & $33(3)$ & 43(4) & $0(3)$ & $-6(3)$ & $-24(3)$ \\
\hline$C(333)$ & 41(3) & $30(3)$ & $42(4)$ & $-6(3)$ & $-12(3)$ & $-8(3)$ \\
\hline$C(334)$ & $37(3)$ & $24(3)$ & $35(3)$ & $-7(2)$ & $-4(3)$ & $-9(2)$ \\
\hline$C(351)$ & $39(3)$ & $24(3)$ & $28(3)$ & $-7(2)$ & $-4(3)$ & $-6(2)$ \\
\hline$C(352)$ & $151(8)$ & $51(5)$ & $79(6)$ & $-42(4)$ & $26(5)$ & $-55(5)$ \\
\hline
\end{tabular}




\begin{tabular}{lllllll}
$\mathrm{C}(353)$ & $61(5)$ & $61(5)$ & $75(5)$ & $-36(4)$ & $-13(4)$ & $10(4)$ \\
$\mathrm{C}(354)$ & $101(6)$ & $50(4)$ & $46(4)$ & $-24(3)$ & $-7(4)$ & $-14(4)$ \\
$\mathrm{C}(531)$ & $54(4)$ & $62(4)$ & $54(4)$ & $-32(4)$ & $16(3)$ & $-39(4)$ \\
$\mathrm{C}(532)$ & $69(5)$ & $86(5)$ & $87(5)$ & $-48(4)$ & $31(4)$ & $-63(4)$ \\
$\mathrm{C}(533)$ & $37(4)$ & $79(5)$ & $91(6)$ & $-45(5)$ & $9(4)$ & $-35(4)$ \\
$\mathrm{C}(534)$ & $74(5)$ & $90(6)$ & $83(5)$ & $-53(5)$ & $47(4)$ & $-61(5)$ \\
$\mathrm{C}(551)$ & $68(4)$ & $43(4)$ & $29(3)$ & $-3(3)$ & $-16(3)$ & $-23(3)$ \\
$\mathrm{C}(552)$ & $128(8)$ & $112(8)$ & $158(9)$ & $-73(7)$ & $-71(7)$ & $-21(6)$ \\
$\mathrm{C}(553)$ & $140(8)$ & $75(6)$ & $84(6)$ & $38(5)$ & $-59(6)$ & $-67(6)$ \\
$\mathrm{C}(554)$ & $79(6)$ & $110(7)$ & $73(6)$ & $20(5)$ & $-47(5)$ & $-46(5)$ \\
$\mathrm{C}(631)$ & $40(3)$ & $41(3)$ & $53(4)$ & $-25(3)$ & $-13(3)$ & $-10(3)$ \\
$\mathrm{C}(632)$ & $48(4)$ & $72(5)$ & $82(5)$ & $-60(4)$ & $-4(4)$ & $-6(4)$ \\
$\mathrm{C}(633)$ & $51(4)$ & $61(4)$ & $48(4)$ & $-23(3)$ & $-1(3)$ & $-12(3)$ \\
$\mathrm{C}(634)$ & $38(3)$ & $40(4)$ & $56(4)$ & $-18(3)$ & $-8(3)$ & $0(3)$ \\
$\mathrm{C}(651)$ & $35(3)$ & $46(3)$ & $37(3)$ & $-14(3)$ & $-5(3)$ & $-19(3)$ \\
$\mathrm{C}(652)$ & $38(3)$ & $49(4)$ & $60(4)$ & $-25(3)$ & $-11(3)$ & $-13(3)$ \\
$\mathrm{C}(653)$ & $55(4)$ & $68(5)$ & $81(5)$ & $-28(4)$ & $5(4)$ & $-41(4)$ \\
$\mathrm{C}(654)$ & $30(3)$ & $105(6)$ & $53(4)$ & $-3(4)$ & $-10(3)$ & $-17(4)$ \\
$\mathrm{C}(731)$ & $48(4)$ & $37(3)$ & $38(4)$ & $-11(3)$ & $-17(3)$ & $3(3)$ \\
$\mathrm{C}(732)$ & $77(5)$ & $49(4)$ & $37(4)$ & $-9(3)$ & $-5(4)$ & $-7(4)$ \\
$\mathrm{C}(733)$ & $79(5)$ & $59(4)$ & $41(4)$ & $-19(3)$ & $-23(4)$ & $-11(4)$ \\
$\mathrm{C}(734)$ & $70(5)$ & $83(5)$ & $44(4)$ & $-15(4)$ & $-22(4)$ & $22(4)$ \\
$\mathrm{C}(751)$ & $37(3)$ & $30(3)$ & $41(3)$ & $-18(3)$ & $5(3)$ & $-17(3)$ \\
$\mathrm{C}(752)$ & $81(5)$ & $58(4)$ & $49(4)$ & $-34(3)$ & $23(4)$ & $-46(4)$ \\
$\mathrm{C}(753)$ & $63(4)$ & $53(4)$ & $38(4)$ & $-11(3)$ & $7(3)$ & $-18(3)$ \\
$\mathrm{C}(754)$ & $49(4)$ & $70(5)$ & $63(5)$ & $-17(4)$ & $9(3)$ & $-34(4)$ \\
\hline & & & & & \\
\hline
\end{tabular}


Table 5. Hydrogen coordinates $\left(\times 10^{4}\right)$ and isotropic displacement parameters $\left(\AA^{2} \times 10^{3}\right)$ for compound 4.

\begin{tabular}{|c|c|c|c|c|}
\hline & $\mathrm{x}$ & $\mathrm{y}$ & $\mathrm{z}$ & $\mathrm{U}(\mathrm{eq})$ \\
\hline $\mathrm{H}(1)$ & $4810(50)$ & $5520(50)$ & $4180(20)$ & $50(20)$ \\
\hline $\mathrm{H}(2)$ & $-1360(50)$ & $1090(50)$ & $9800(20)$ & $50(20)$ \\
\hline $\mathrm{H}(14)$ & -220 & 6465 & 5632 & 33 \\
\hline $\mathrm{H}(24)$ & 6623 & 323 & 4910 & 31 \\
\hline $\mathrm{H}(34)$ & 7198 & 921 & 7600 & 32 \\
\hline $\mathrm{H}(41 \mathrm{~A})$ & 3250 & 2447 & 4569 & 49 \\
\hline $\mathrm{H}(41 \mathrm{~B})$ & 4404 & 2537 & 4310 & 49 \\
\hline $\mathrm{H}(42 \mathrm{~A})$ & 3227 & 2600 & 3681 & 76 \\
\hline $\mathrm{H}(42 \mathrm{~B})$ & 3929 & 3313 & 3495 & 76 \\
\hline $\mathrm{H}(43 \mathrm{~A})$ & 1671 & 3985 & 3815 & 49 \\
\hline $\mathrm{H}(43 \mathrm{~B})$ & 2347 & 4676 & 3455 & 49 \\
\hline $\mathrm{H}(44 \mathrm{~A})$ & 2166 & 5296 & 4218 & 57 \\
\hline $\mathrm{H}(44 \mathrm{~B})$ & 1580 & 4535 & 4585 & 57 \\
\hline $\mathrm{H}(52 \mathrm{~A})$ & 6036 & 3102 & 3611 & 54 \\
\hline $\mathrm{H}(52 \mathrm{~B})$ & 7246 & 3047 & 3370 & 54 \\
\hline $\mathrm{H}(52 \mathrm{C})$ & 6568 & 3575 & 3918 & 54 \\
\hline $\mathrm{H}(54)$ & 2259 & 1316 & 7693 & 67 \\
\hline $\mathrm{H}(64)$ & 350 & 2866 & 11524 & 42 \\
\hline $\mathrm{H}(74)$ & -6120 & 3756 & 10686 & 42 \\
\hline $\mathrm{H}(81 \mathrm{~A})$ & -400 & 3620 & 10179 & 85 \\
\hline $\mathrm{H}(81 \mathrm{~B})$ & -1463 & 3545 & 10072 & 85 \\
\hline $\mathrm{H}(82 \mathrm{~A})$ & -1008 & 5212 & 9689 & 129 \\
\hline $\mathrm{H}(82 \mathrm{~B})$ & -1775 & 4941 & 9415 & 129 \\
\hline $\mathrm{H}(83 \mathrm{~A})$ & -419 & 4896 & 8663 & 95 \\
\hline $\mathrm{H}(83 \mathrm{~B})$ & 503 & 4485 & 9048 & 95 \\
\hline $\mathrm{H}(84 \mathrm{~A})$ & 814 & 3010 & 8789 & 87 \\
\hline $\mathrm{H}(84 \mathrm{~B})$ & -448 & 3350 & 8765 & 87 \\
\hline $\mathrm{H}(85 \mathrm{~A})$ & -1414 & 4425 & 8799 & 74 \\
\hline $\mathrm{H}(85 \mathrm{~B})$ & -491 & 4902 & 8635 & 74 \\
\hline $\mathrm{H}(13 \mathrm{~A})$ & 3068 & 3781 & 6040 & 56 \\
\hline
\end{tabular}




\begin{tabular}{|c|c|c|c|c|}
\hline $\mathrm{H}(13 \mathrm{~B})$ & 2695 & 3879 & 6690 & 56 \\
\hline $\mathrm{H}(13 \mathrm{C})$ & 3068 & 4742 & 6263 & 56 \\
\hline $\mathrm{H}(13 \mathrm{D})$ & 230 & 4581 & 6189 & 67 \\
\hline $\mathrm{H}(13 \mathrm{E})$ & 1033 & 3694 & 6587 & 67 \\
\hline $\mathrm{H}(13 \mathrm{~F})$ & 1381 & 3757 & 5924 & 67 \\
\hline $\mathrm{H}(13 \mathrm{G})$ & 1242 & 6042 & 6704 & 70 \\
\hline $\mathrm{H}(13 \mathrm{H})$ & 929 & 5086 & 7056 & 70 \\
\hline $\mathrm{H}(13 \mathrm{I})$ & 145 & 5976 & 6651 & 70 \\
\hline $\mathrm{H}(15 \mathrm{~A})$ & 959 & 8767 & 4460 & 53 \\
\hline $\mathrm{H}(15 \mathrm{~B})$ & -41 & 8963 & 4971 & 53 \\
\hline $\mathrm{H}(15 \mathrm{C})$ & -268 & 9372 & 4344 & 53 \\
\hline $\mathrm{H}(15 \mathrm{D})$ & 1375 & 7431 & 3865 & 53 \\
\hline $\mathrm{H}(15 \mathrm{E})$ & 174 & 8087 & 3718 & 53 \\
\hline $\mathrm{H}(15 \mathrm{~F})$ & 567 & 6854 & 3951 & 53 \\
\hline $\mathrm{H}(15 \mathrm{G})$ & -1043 & 7198 & 4741 & 61 \\
\hline $\mathrm{H}(15 \mathrm{H})$ & -1446 & 8413 & 4468 & 61 \\
\hline $\mathrm{H}(15 \mathrm{I})$ & -1302 & 8081 & 5109 & 61 \\
\hline $\mathrm{H}(23 \mathrm{~A})$ & 5345 & -483 & 5554 & 51 \\
\hline $\mathrm{H}(23 \mathrm{~B})$ & 5013 & -628 & 6219 & 51 \\
\hline $\mathrm{H}(23 \mathrm{C})$ & 6139 & -515 & 5931 & 51 \\
\hline $\mathrm{H}(23 \mathrm{D})$ & 5521 & 1035 & 6469 & 49 \\
\hline $\mathrm{H}(23 \mathrm{E})$ & 4437 & 825 & 6733 & 49 \\
\hline $\mathrm{H}(23 \mathrm{~F})$ & 4348 & 1973 & 6422 & 49 \\
\hline $\mathrm{H}(23 \mathrm{G})$ & 3265 & 2045 & 5731 & 53 \\
\hline $\mathrm{H}(23 \mathrm{H})$ & 3216 & 975 & 6099 & 53 \\
\hline $\mathrm{H}(23 \mathrm{I})$ & 3639 & 1028 & 5440 & 53 \\
\hline $\mathrm{H}(25 \mathrm{~A})$ & 7632 & 392 & 3985 & 57 \\
\hline $\mathrm{H}(25 \mathrm{~B})$ & 7936 & 1078 & 3414 & 57 \\
\hline $\mathrm{H}(25 \mathrm{C})$ & 6700 & 1199 & 3633 & 57 \\
\hline $\mathrm{H}(25 \mathrm{D})$ & 8158 & 2376 & 4382 & 54 \\
\hline $\mathrm{H}(25 \mathrm{E})$ & 8793 & 1774 & 3853 & 54 \\
\hline $\mathrm{H}(25 \mathrm{~F})$ & 8544 & 1126 & 4450 & 54 \\
\hline $\mathrm{H}(33 \mathrm{~A})$ & 7163 & -880 & 7458 & 62 \\
\hline $\mathrm{H}(33 \mathrm{~B})$ & 7822 & -1805 & 7084 & 62 \\
\hline $\mathrm{H}(33 \mathrm{C})$ & 6766 & -821 & 6900 & 62 \\
\hline $\mathrm{H}(33 \mathrm{D})$ & 9607 & -477 & 6800 & 58 \\
\hline
\end{tabular}




\begin{tabular}{|c|c|c|c|c|}
\hline $\mathrm{H}(33 \mathrm{E})$ & 9563 & -1616 & 6984 & 58 \\
\hline $\mathrm{H}(33 \mathrm{~F})$ & 8937 & -750 & 7394 & 58 \\
\hline $\mathrm{H}(33 \mathrm{G})$ & 7815 & -394 & 5964 & 50 \\
\hline $\mathrm{H}(33 \mathrm{H})$ & 8794 & -1467 & 6148 & 50 \\
\hline $\mathrm{H}(33 \mathrm{I})$ & 8989 & -400 & 5934 & 50 \\
\hline $\mathrm{H}(35 \mathrm{~A})$ & 5924 & 4721 & 7189 & 137 \\
\hline $\mathrm{H}(35 \mathrm{~B})$ & 6420 & 4153 & 6644 & 137 \\
\hline $\mathrm{H}(35 \mathrm{C})$ & 7133 & 3820 & 7123 & 137 \\
\hline $\mathrm{H}(35 \mathrm{D})$ & 4441 & 3171 & 7437 & 110 \\
\hline $\mathrm{H}(35 \mathrm{E})$ & 4690 & 3889 & 6867 & 110 \\
\hline $\mathrm{H}(35 \mathrm{~F})$ & 4336 & 4330 & 7447 & 110 \\
\hline $\mathrm{H}(35 \mathrm{G})$ & 6730 & 2749 & 7992 & 104 \\
\hline $\mathrm{H}(35 \mathrm{H})$ & 5767 & 2359 & 8078 & 104 \\
\hline $\mathrm{H}(35 \mathrm{I})$ & 5484 & 3556 & 8123 & 104 \\
\hline $\mathrm{H}(53 \mathrm{~A})$ & 2801 & 2105 & 9101 & 107 \\
\hline $\mathrm{H}(53 \mathrm{~B})$ & 2902 & 2671 & 8468 & 107 \\
\hline $\mathrm{H}(53 \mathrm{C})$ & 3948 & 2127 & 8770 & 107 \\
\hline $\mathrm{H}(53 \mathrm{D})$ & 3729 & 101 & 9305 & 94 \\
\hline $\mathrm{H}(53 \mathrm{E})$ & 4847 & 191 & 8975 & 94 \\
\hline $\mathrm{H}(53 \mathrm{~F})$ & 4421 & -553 & 8800 & 94 \\
\hline $\mathrm{H}(53 \mathrm{G})$ & 4916 & 1105 & 7950 & 115 \\
\hline $\mathrm{H}(53 \mathrm{H})$ & 3817 & 1623 & 7692 & 115 \\
\hline $\mathrm{H}(53 \mathrm{I})$ & 4434 & 368 & 7814 & 115 \\
\hline $\mathrm{H}(55 \mathrm{~A})$ & 1086 & 321 & 7380 & 185 \\
\hline $\mathrm{H}(55 \mathrm{~B})$ & -144 & 410 & 7506 & 185 \\
\hline $\mathrm{H}(55 \mathrm{C})$ & 674 & -348 & 7928 & 185 \\
\hline $\mathrm{H}(55 \mathrm{D})$ & 437 & 2304 & 7350 & 138 \\
\hline $\mathrm{H}(55 \mathrm{E})$ & -496 & 2839 & 7844 & 138 \\
\hline $\mathrm{H}(55 \mathrm{~F})$ & -765 & 2350 & 7417 & 138 \\
\hline $\mathrm{H}(55 \mathrm{G})$ & -1482 & 1333 & 8219 & 126 \\
\hline $\mathrm{H}(55 \mathrm{H})$ & -1167 & 1821 & 8628 & 126 \\
\hline $\mathrm{H}(55 \mathrm{I})$ & -739 & 570 & 8673 & 126 \\
\hline $\mathrm{H}(63 \mathrm{~A})$ & -1171 & 3329 & 12297 & 99 \\
\hline $\mathrm{H}(63 \mathrm{~B})$ & -2366 & 4248 & 12294 & 99 \\
\hline $\mathrm{H}(63 \mathrm{C})$ & -1379 & 4319 & 11810 & 99 \\
\hline $\mathrm{H}(63 \mathrm{D})$ & -2733 & 2222 & 11907 & 84 \\
\hline
\end{tabular}




\begin{tabular}{|c|c|c|c|c|}
\hline $\mathrm{H}(63 \mathrm{E})$ & -3139 & 2996 & 12360 & 84 \\
\hline $\mathrm{H}(63 \mathrm{~F})$ & -1980 & 2017 & 12348 & 84 \\
\hline $\mathrm{H}(63 \mathrm{G})$ & -2377 & 4495 & 11069 & 73 \\
\hline $\mathrm{H}(63 \mathrm{H})$ & -3392 & 4513 & 11554 & 73 \\
\hline $\mathrm{H}(63 \mathrm{I})$ & -2936 & 3695 & 11121 & 73 \\
\hline $\mathrm{H}(65 \mathrm{~A})$ & 2135 & 214 & 10430 & 70 \\
\hline $\mathrm{H}(65 \mathrm{~B})$ & 3222 & 434 & 10276 & 70 \\
\hline $\mathrm{H}(65 \mathrm{C})$ & 2187 & 1217 & 9988 & 70 \\
\hline $\mathrm{H}(65 \mathrm{D})$ & 2091 & 2747 & 10275 & 93 \\
\hline $\mathrm{H}(65 \mathrm{E})$ & 3164 & 2047 & 10547 & 93 \\
\hline $\mathrm{H}(65 \mathrm{~F})$ & 2077 & 2769 & 10912 & 93 \\
\hline $\mathrm{H}(65 \mathrm{G})$ & 2198 & 1160 & 11620 & 102 \\
\hline $\mathrm{H}(65 \mathrm{H})$ & 3280 & 495 & 11230 & 102 \\
\hline $\mathrm{H}(65 \mathrm{I})$ & 2283 & 132 & 11427 & 102 \\
\hline $\mathrm{H}(73 \mathrm{~A})$ & -3615 & 4188 & 9519 & 91 \\
\hline $\mathrm{H}(73 \mathrm{~B})$ & -3342 & 3544 & 9020 & 91 \\
\hline $\mathrm{H}(73 \mathrm{C})$ & -2778 & 2977 & 9560 & 91 \\
\hline $\mathrm{H}(73 \mathrm{D})$ & -3478 & 1583 & 9629 & 91 \\
\hline $\mathrm{H}(73 \mathrm{E})$ & -4073 & 2231 & 9105 & 91 \\
\hline $\mathrm{H}(73 \mathrm{~F})$ & -4760 & 1914 & 9683 & 91 \\
\hline $\mathrm{H}(73 \mathrm{G})$ & -6050 & 3826 & 9714 & 122 \\
\hline $\mathrm{H}(73 \mathrm{H})$ & -5378 & 4079 & 9116 & 122 \\
\hline $\mathrm{H}(73 \mathrm{I})$ & -5549 & 4690 & 9613 & 122 \\
\hline $\mathrm{H}(75 \mathrm{~A})$ & -4992 & 3920 & 11976 & 88 \\
\hline $\mathrm{H}(75 \mathrm{~B})$ & -6159 & 4073 & 12355 & 88 \\
\hline $\mathrm{H}(75 \mathrm{C})$ & -6080 & 4593 & 11713 & 88 \\
\hline $\mathrm{H}(75 \mathrm{D})$ & -5067 & 1396 & 12131 & 83 \\
\hline $\mathrm{H}(75 \mathrm{E})$ & -5623 & 2128 & 12611 & 83 \\
\hline $\mathrm{H}(75 \mathrm{~F})$ & -4433 & 1993 & 12270 & 83 \\
\hline $\mathrm{H}(75 \mathrm{G})$ & -7173 & 3737 & 11569 & 89 \\
\hline $\mathrm{H}(75 \mathrm{H})$ & -7244 & 3214 & 12211 & 89 \\
\hline $\mathrm{H}(75 \mathrm{I})$ & -6810 & 2488 & 11733 & 89 \\
\hline
\end{tabular}


Table for Compound 5: $\left[\left\{\operatorname{Sr}_{2}(\mathbf{O M e s})_{2}(\mu \text {-OMes })_{2}\left(\eta^{2} \text {-en }\right)_{3}\left(\eta^{1} \text {-en }\right)\left(\mathrm{H}_{2} \mathrm{O}\right)\right\}_{2}(\mu\right.$-en $\left.)\right] \cdot 3 \mathrm{en}$ 
Table 1. Crystal data and structure refinement for compound 5.

Identification code

Empirical formula

Formula weight

Temperature

Wavelength

Crystal system

Space group

Unit cell dimensions

Volume

Z

Density (calculated)

Absorption coefficient

$\mathrm{F}(000)$

Crystal size

Theta range for data collection

Index ranges

Reflections collected

Independent reflections

Completeness to theta $=25.00^{\circ}$

Absorption correction

Max. and min. transmission

Refinement method

Data / restraints / parameters

Goodness-of-fit on $\mathrm{F}^{2}$

Final $\mathrm{R}$ indices [I $>2 \operatorname{sigma}(\mathrm{I})]$

$\mathrm{R}$ indices (all data)

Largest diff. peak and hole $\mathrm{sad}$

C95 H184 N23 O10 Sr4

2159.13

93(2) K

$0.71073 \AA$

Monoclinic

$\mathrm{P} 2(1) / \mathrm{c}$

$\mathrm{a}=25.559(6) \AA$

$\alpha=90^{\circ}$.

$\mathrm{b}=19.512(5) \AA$

$\beta=111.880(5)^{\circ}$.

$\mathrm{c}=24.723(6) \AA$

$\gamma=90^{\circ}$.

11441(5) $\AA^{3}$

4

$1.253 \mathrm{Mg} / \mathrm{m}^{3}$

$1.915 \mathrm{~mm}^{-1}$

4588

$0.72 \times 0.64 \times 0.30 \mathrm{~mm}^{3}$

1.78 to $25.00^{\circ}$.

$-30<=\mathrm{h}<=30,-23<=\mathrm{k}<=23,-29<=1<=29$

72977

$17385[\mathrm{R}(\mathrm{int})=0.0919]$

$86.2 \%$

Semi-empirical from equivalents

0.5973 and 0.3393

Full-matrix least-squares on $\mathrm{F}^{2}$

17385 / 4 / 1222

0.876

$\mathrm{R} 1=0.0478, \mathrm{wR} 2=0.1113$

$\mathrm{R} 1=0.0864, \mathrm{wR} 2=0.1164$

0.466 and -0.460 e. $\AA^{-3}$ 


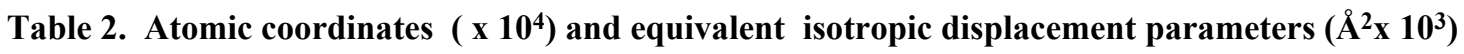
for compound 5. $U(e q)$ is defined as one third of the trace of the orthogonalized $U^{i j}$ tensor.

\begin{tabular}{|c|c|c|c|c|}
\hline & $\mathrm{x}$ & $\mathrm{y}$ & $\mathrm{z}$ & $\mathrm{U}(\mathrm{eq})$ \\
\hline $\operatorname{Sr}(1)$ & $4766(1)$ & $1466(1)$ & $8299(1)$ & $17(1)$ \\
\hline $\operatorname{Sr}(3)$ & $9047(1)$ & $7236(1)$ & $6639(1)$ & $19(1)$ \\
\hline $\operatorname{Sr}(2)$ & $5955(1)$ & 2789(1) & $8510(1)$ & $17(1)$ \\
\hline $\operatorname{Sr}(4)$ & $9216(1)$ & $5785(1)$ & $7789(1)$ & $20(1)$ \\
\hline $\mathrm{O}(1)$ & $5445(1)$ & $1822(2)$ & $7849(1)$ & $18(1)$ \\
\hline $\mathrm{C}(1)$ & $5442(2)$ & 1544(3) & $7355(2)$ & $17(1)$ \\
\hline$C(2)$ & $5300(2)$ & 1941(3) & $6841(2)$ & $18(1)$ \\
\hline$C(3)$ & $5314(2)$ & $1652(3)$ & $6332(2)$ & $25(1)$ \\
\hline$C(4)$ & $5454(2)$ & $970(3)$ & $6300(2)$ & $30(1)$ \\
\hline$C(5)$ & $5570(2)$ & $570(3)$ & $6797(2)$ & $21(1)$ \\
\hline$C(6)$ & $5568(2)$ & $841(2)$ & $7328(2)$ & $16(1)$ \\
\hline$C(7)$ & $5123(2)$ & 2681(3) & $6853(2)$ & $27(1)$ \\
\hline $\mathrm{C}(8)$ & $5488(3)$ & $669(3)$ & $5757(2)$ & $39(2)$ \\
\hline $\mathrm{C}(9)$ & $5683(2)$ & $386(3)$ & $7842(2)$ & $29(1)$ \\
\hline $\mathrm{O}(2)$ & $5019(1)$ & $2687(2)$ & $8600(1)$ & $20(1)$ \\
\hline$C(10)$ & $4697(2)$ & $3040(3)$ & $8814(2)$ & $20(1)$ \\
\hline$C(11)$ & $4782(2)$ & $3000(3)$ & $9416(2)$ & $23(1)$ \\
\hline$C(12)$ & $4419(2)$ & $3354(3)$ & $9619(2)$ & $27(1)$ \\
\hline$C(13)$ & $3973(2)$ & $3756(3)$ & $9261(2)$ & $28(1)$ \\
\hline$C(14)$ & $3902(2)$ & $3782(3)$ & $8675(2)$ & $25(1)$ \\
\hline$C(15)$ & $4240(2)$ & $3438(2)$ & $8445(2)$ & $21(1)$ \\
\hline$C(16)$ & $5249(2)$ & $2570(3)$ & $9825(2)$ & $28(1)$ \\
\hline$C(17)$ & $3600(3)$ & $4149(3)$ & 9501(3) & $42(2)$ \\
\hline$C(18)$ & $4125(2)$ & $3454(3)$ & $7799(2)$ & $31(1)$ \\
\hline $\mathrm{O}(3)$ & $6507(1)$ & $2042(2)$ & $9298(2)$ & $28(1)$ \\
\hline$C(19)$ & $7038(2)$ & 1937(3) & $9638(2)$ & $27(1)$ \\
\hline $\mathrm{C}(20)$ & $7279(2)$ & 2299(3) & $10169(2)$ & $29(1)$ \\
\hline $\mathrm{C}(21)$ & $7833(2)$ & 2181(3) & $10526(3)$ & $36(1)$ \\
\hline$C(22)$ & $8168(2)$ & $1735(3)$ & $10378(3)$ & $41(2)$ \\
\hline$C(23)$ & $7942(2)$ & 1386(3) & $9857(3)$ & $36(2)$ \\
\hline$C(24)$ & $7386(2)$ & $1474(3)$ & $9487(2)$ & $30(1)$ \\
\hline
\end{tabular}




\begin{tabular}{|c|c|c|c|c|}
\hline$C(25)$ & $6926(3)$ & 2808(3) & $10333(2)$ & $38(2)$ \\
\hline$C(26)$ & $8790(3)$ & $1606(4)$ & 10781(3) & $67(2)$ \\
\hline $\mathrm{C}(27)$ & $7134(2)$ & 1093(3) & $8923(3)$ & $39(2)$ \\
\hline $\mathrm{O}(4)$ & $4315(1)$ & $361(2)$ & $7990(2)$ & $26(1)$ \\
\hline $\mathrm{C}(28)$ & $4051(2)$ & $-158(3)$ & $8107(2)$ & $22(1)$ \\
\hline C(29) & $3471(2)$ & $-259(3)$ & $7792(2)$ & $20(1)$ \\
\hline$C(30)$ & $3195(2)$ & $-815(3)$ & 7921(2) & $26(1)$ \\
\hline $\mathrm{C}(31)$ & $3462(2)$ & $-1282(3)$ & $8349(2)$ & $26(1)$ \\
\hline$C(32)$ & $4032(2)$ & $-1188(3)$ & $8652(2)$ & $25(1)$ \\
\hline C(33) & $4330(2)$ & $-650(3)$ & $8543(2)$ & $22(1)$ \\
\hline$C(34)$ & $3160(2)$ & $234(3)$ & $7302(2)$ & $27(1)$ \\
\hline $\mathrm{C}(35)$ & $3160(3)$ & $-1894(3)$ & $8466(2)$ & $38(2)$ \\
\hline $\mathrm{O}(5)$ & $5682(2)$ & $1127(2)$ & $9105(2)$ & $24(1)$ \\
\hline $\mathrm{N}(1)$ & $5907(2)$ & $3889(2)$ & $9228(2)$ & $25(1)$ \\
\hline $\mathrm{N}(2)$ & $5657(2)$ & $4043(2)$ & $7952(2)$ & $30(1)$ \\
\hline$C(36)$ & $4958(2)$ & $-589(3)$ & $8863(2)$ & $30(1)$ \\
\hline$C(37)$ & $5594(2)$ & $4460(3)$ & $8876(2)$ & $31(1)$ \\
\hline $\mathrm{N}(3)$ & $7031(2)$ & $3344(2)$ & $8957(2)$ & $25(1)$ \\
\hline $\mathrm{N}(4)$ & $6592(2)$ & $2696(2)$ & $7816(2)$ & $30(1)$ \\
\hline$C(38)$ & $5789(2)$ & $4617(3)$ & $8382(2)$ & $32(1)$ \\
\hline$C(39)$ & $7288(2)$ & $3456(3)$ & $8525(2)$ & $25(1)$ \\
\hline $\mathrm{N}(5)$ & $3726(2)$ & $1842(2)$ & $8298(2)$ & $27(1)$ \\
\hline $\mathrm{N}(6)$ & $4519(2)$ & $1058(2)$ & $9260(2)$ & $26(1)$ \\
\hline $\mathrm{C}(40)$ & $7198(2)$ & $2828(3)$ & $8139(2)$ & $27(1)$ \\
\hline $\mathrm{C}(41)$ & $3558(2)$ & 1394(3) & $8688(2)$ & $26(1)$ \\
\hline $\mathrm{O}(6)$ & $9653(1)$ & $6196(2)$ & $7097(2)$ & $24(1)$ \\
\hline $\mathrm{C}(42)$ & $4013(2)$ & $1387(3)$ & $9288(2)$ & $26(1)$ \\
\hline $\mathrm{C}(46)$ & $10125(2)$ & $5970(3)$ & $7040(2)$ & $24(1)$ \\
\hline $\mathrm{C}(47)$ & $10158(2)$ & $5299(3)$ & $6839(2)$ & $25(1)$ \\
\hline $\mathrm{C}(48)$ & $10632(2)$ & $5107(3)$ & $6746(2)$ & $35(1)$ \\
\hline $\mathrm{C}(49)$ & $11097(2)$ & $5531(3)$ & $6859(3)$ & $40(2)$ \\
\hline $\mathrm{C}(50)$ & $11070(2)$ & $6163(3)$ & $7082(2)$ & $34(1)$ \\
\hline $\mathrm{C}(51)$ & $10597(2)$ & $6395(3)$ & $7184(2)$ & $26(1)$ \\
\hline$C(52)$ & $9670(2)$ & 4811(3) & $6724(2)$ & $35(1)$ \\
\hline $\mathrm{C}(53)$ & $11606(3)$ & $5283(4)$ & $6731(3)$ & $67(2)$ \\
\hline $\mathrm{O}(7)$ & $8969(1)$ & $7017(2)$ & $7610(1)$ & $22(1)$ \\
\hline
\end{tabular}




\begin{tabular}{|c|c|c|c|c|}
\hline$C(54)$ & $10598(2)$ & $7086(3)$ & $7451(2)$ & $37(2)$ \\
\hline $\mathrm{C}(55)$ & $8765(2)$ & $7400(3)$ & $7935(2)$ & $23(1)$ \\
\hline$C(56)$ & $8180(2)$ & $7422(3)$ & $7811(2)$ & $23(1)$ \\
\hline $\mathrm{C}(57)$ & 7984(2) & $7828(3)$ & $8156(2)$ & $32(1)$ \\
\hline $\mathrm{C}(58)$ & $8323(3)$ & $8212(3)$ & $8611(3)$ & $38(2)$ \\
\hline C(59) & $8902(2)$ & $8177(3)$ & $8738(2)$ & $27(1)$ \\
\hline$C(60)$ & $9128(2)$ & $7776(3)$ & $8409(2)$ & $22(1)$ \\
\hline $\mathrm{C}(61)$ & $7774(2)$ & $7007(3)$ & $7312(2)$ & $32(1)$ \\
\hline$C(62)$ & $8102(3)$ & $8630(3)$ & 8994(3) & $52(2)$ \\
\hline $\mathrm{O}(8)$ & $8200(1)$ & $6619(2)$ & $6084(1)$ & $26(1)$ \\
\hline$C(63)$ & $9748(2)$ & $7762(3)$ & $8560(2)$ & $33(1)$ \\
\hline$C(64)$ & $7796(2)$ & $6441(3)$ & $5581(2)$ & $20(1)$ \\
\hline$C(65)$ & $7861(2)$ & $5883(3)$ & $5257(2)$ & $22(1)$ \\
\hline$C(66)$ & $7423(2)$ & $5681(3)$ & $4744(2)$ & $22(1)$ \\
\hline$C(67)$ & $6923(2)$ & $6040(3)$ & $4526(2)$ & $26(1)$ \\
\hline$C(68)$ & $6867(2)$ & $6611(3)$ & $4837(2)$ & $24(1)$ \\
\hline $\mathrm{C}(69)$ & $7290(2)$ & $6827(3)$ & $5350(2)$ & $23(1)$ \\
\hline$C(70)$ & $8416(2)$ & $5506(3)$ & $5455(2)$ & $28(1)$ \\
\hline $\mathrm{C}(71)$ & $6455(2)$ & $5832(3)$ & $3962(2)$ & $41(2)$ \\
\hline $\mathrm{O}(9)$ & $9548(1)$ & $4642(2)$ & $8172(1)$ & $26(1)$ \\
\hline$C(72)$ & $7215(2)$ & $7468(3)$ & $5656(2)$ & $30(1)$ \\
\hline$C(73)$ & $9378(2)$ & $4136(3)$ & $8421(2)$ & $26(1)$ \\
\hline$C(74)$ & $9629(2)$ & $4030(3)$ & $9025(2)$ & $25(1)$ \\
\hline$C(75)$ & $9446(2)$ & $3496(3)$ & $9278(2)$ & $33(1)$ \\
\hline$C(76)$ & $9021(2)$ & $3049(3)$ & $8966(3)$ & $34(1)$ \\
\hline$C(77)$ & $8780(2)$ & $3160(3)$ & $8369(3)$ & $32(1)$ \\
\hline $\mathrm{C}(78)$ & $8934(2)$ & $3684(3)$ & $8085(2)$ & $26(1)$ \\
\hline$C(79)$ & $10099(2)$ & $4496(3)$ & $9377(2)$ & $34(1)$ \\
\hline $\mathrm{C}(80)$ & $8858(3)$ & $2445(3)$ & $9265(3)$ & $50(2)$ \\
\hline $\mathrm{C}(81)$ & $8667(2)$ & $3775(3)$ & $7439(2)$ & $33(1)$ \\
\hline $\mathrm{O}(10)$ & $8353(2)$ & $5556(2)$ & $6854(2)$ & $30(1)$ \\
\hline $\mathrm{N}(7)$ & $8385(2)$ & $8323(2)$ & $6737(2)$ & $26(1)$ \\
\hline $\mathrm{N}(8)$ & $9602(2)$ & $8485(2)$ & $7017(2)$ & $38(1)$ \\
\hline$C(82)$ & $8716(2)$ & 8903(3) & $7074(2)$ & $33(1)$ \\
\hline $\mathrm{C}(83)$ & $9198(2)$ & 9064(3) & $6881(2)$ & $32(1)$ \\
\hline $\mathrm{N}(9)$ & $8623(2)$ & $7831(2)$ & $5535(2)$ & $27(1)$ \\
\hline
\end{tabular}




\begin{tabular}{|c|c|c|c|c|}
\hline $\mathrm{N}(10)$ & $9709(2)$ & $7159(2)$ & $5967(2)$ & $36(1)$ \\
\hline $\mathrm{C}(84)$ & $8998(2)$ & $7845(3)$ & $5222(2)$ & $31(1)$ \\
\hline $\mathrm{C}(85)$ & $9365(3)$ & $7232(3)$ & $5343(2)$ & $40(2)$ \\
\hline $\mathrm{N}(11)$ & $9223(2)$ & $6155(2)$ & $8900(2)$ & $31(1)$ \\
\hline $\mathrm{N}(12)$ & $8344(2)$ & $5324(2)$ & $8083(2)$ & $34(1)$ \\
\hline$C(86)$ & $8843(2)$ & $5730(3)$ & $9073(2)$ & $33(1)$ \\
\hline$C(87)$ & $8286(2)$ & $5666(3)$ & $8586(2)$ & $38(2)$ \\
\hline $\mathrm{N}(1 \mathrm{~S})$ & $4076(2)$ & $1578(2)$ & $7092(2)$ & $26(1)$ \\
\hline $\mathrm{N}(2 \mathrm{~S})$ & $2709(2)$ & 2027(3) & $5821(3)$ & $79(2)$ \\
\hline $\mathrm{C}(1 \mathrm{~S})$ & $3577(2)$ & 1975(3) & $6738(2)$ & $34(1)$ \\
\hline$C(2 S)$ & $3239(3)$ & $1658(4)$ & $6156(3)$ & $60(2)$ \\
\hline $\mathrm{N}(3 \mathrm{~S})$ & $10382(2)$ & $5831(2)$ & $8464(2)$ & $36(1)$ \\
\hline $\mathrm{N}(4 \mathrm{~S})$ & $11590(2)$ & $6315(3)$ & $9837(2)$ & $67(2)$ \\
\hline$C(3 S)$ & $10699(5)$ & $6206(5)$ & $9015(5)$ & $28(3)$ \\
\hline$C(4 S)$ & $11301(5)$ & $5947(5)$ & $9269(5)$ & $43(4)$ \\
\hline $\mathrm{N}\left(3 \mathrm{~S}^{\prime}\right)$ & $10382(2)$ & $5831(2)$ & $8464(2)$ & $36(1)$ \\
\hline $\mathrm{N}\left(4 \mathrm{~S}^{\prime}\right)$ & $11590(2)$ & $6315(3)$ & $9837(2)$ & $67(2)$ \\
\hline$C\left(3 S^{\prime}\right)$ & $10970(8)$ & $6071(10)$ & $8865(9)$ & $43(6)$ \\
\hline$C\left(4 S^{\prime}\right)$ & 10942(9) & $6150(10)$ & $9422(9)$ & $48(7)$ \\
\hline $\mathrm{N}(5 \mathrm{~S})$ & $7248(2)$ & $10495(3)$ & $4928(2)$ & $54(2)$ \\
\hline $\mathrm{N}(6 \mathrm{~S})$ & $7840(2)$ & $9207(3)$ & $5504(2)$ & $45(1)$ \\
\hline $\mathrm{C}(5 \mathrm{~S})$ & $7330(4)$ & 9899(4) & $4625(3)$ & $75(3)$ \\
\hline$C(6 S)$ & $7868(3)$ & $9477(4)$ & $4985(3)$ & $68(2)$ \\
\hline $\mathrm{N}(7 \mathrm{~S})$ & $7696(3)$ & $545(3)$ & $6447(3)$ & $71(2)$ \\
\hline $\mathrm{N}(8 \mathrm{~S})$ & $6849(2)$ & $1288(3)$ & $7275(2)$ & $62(2)$ \\
\hline$C(7 S)$ & $7259(3)$ & $577(4)$ & 6693(3) & $55(2)$ \\
\hline$C(8 S)$ & $7255(3)$ & $1254(3)$ & 7004(3) & $41(2)$ \\
\hline $\mathrm{N}(9 \mathrm{~S})$ & $7615(2)$ & $4558(2)$ & $6081(2)$ & $34(1)$ \\
\hline $\mathrm{N}(10 \mathrm{~S})$ & $6059(2)$ & $4689(2)$ & $5120(2)$ & $38(1)$ \\
\hline $\mathrm{C}(9 \mathrm{~S})$ & $7047(2)$ & $4845(3)$ & $5842(2)$ & $22(1)$ \\
\hline $\mathrm{C}(10 \mathrm{~S})$ & $6644(2)$ & $4445(3)$ & $5335(2)$ & $23(1)$ \\
\hline $\mathrm{N}(11 \mathrm{~S})$ & $-217(4)$ & $844(4)$ & $9669(4)$ & $143(4)$ \\
\hline $\mathrm{C}(11 \mathrm{~S})$ & $176(4)$ & $309(4)$ & $9934(4)$ & $93(3)$ \\
\hline
\end{tabular}


Table 3. Bond lengths $[\AA ̊]$ and angles $\left[^{\circ}\right]$ for compound 5.

\begin{tabular}{|c|c|c|c|}
\hline $\operatorname{Sr}(1)-O(4)$ & $2.429(3)$ & $\mathrm{C}(10)-\mathrm{C}(15)$ & $1.416(7)$ \\
\hline $\operatorname{Sr}(1)-O(1)$ & $2.483(3)$ & $C(10)-C(11)$ & $1.424(7)$ \\
\hline $\operatorname{Sr}(1)-O(2)$ & $2.507(3)$ & $\mathrm{C}(11)-\mathrm{C}(12)$ & $1.389(7)$ \\
\hline $\operatorname{Sr}(1)-O(5)$ & $2.532(4)$ & $C(11)-C(16)$ & $1.502(7)$ \\
\hline $\operatorname{Sr}(3)-O(8)$ & $2.409(3)$ & $\mathrm{C}(12)-\mathrm{C}(13)$ & $1.396(8)$ \\
\hline $\operatorname{Sr}(3)-O(7)$ & $2.520(3)$ & $\mathrm{C}(13)-\mathrm{C}(14)$ & $1.392(7)$ \\
\hline $\operatorname{Sr}(3)-O(6)$ & $2.546(3)$ & $\mathrm{C}(13)-\mathrm{C}(17)$ & $1.507(7)$ \\
\hline $\operatorname{Sr}(3)-N(7)$ & $2.781(4)$ & $\mathrm{C}(14)-\mathrm{C}(15)$ & $1.373(7)$ \\
\hline $\operatorname{Sr}(3)-N(10)$ & $2.783(4)$ & $C(15)-C(18)$ & $1.512(7)$ \\
\hline $\operatorname{Sr}(3)-N(9)$ & $2.787(4)$ & $\mathrm{O}(3)-\mathrm{C}(19)$ & $1.319(6)$ \\
\hline $\operatorname{Sr}(3)-\mathrm{N}(8)$ & $2.799(4)$ & $C(19)-C(24)$ & $1.412(7)$ \\
\hline $\operatorname{Sr}(2)-O(3)$ & $2.421(3)$ & $\mathrm{C}(19)-\mathrm{C}(20)$ & $1.412(8)$ \\
\hline $\mathrm{Sr}(2)-\mathrm{O}(2)$ & $2.492(3)$ & $C(20)-C(21)$ & $1.381(7)$ \\
\hline $\operatorname{Sr}(2)-O(1)$ & $2.519(3)$ & $\mathrm{C}(20)-\mathrm{C}(25)$ & $1.496(7)$ \\
\hline $\mathrm{Sr}(2)-\mathrm{N}(2)$ & $2.771(4)$ & $C(21)-C(22)$ & $1.361(8)$ \\
\hline $\operatorname{Sr}(2)-N(1)$ & $2.817(4)$ & $\mathrm{C}(22)-\mathrm{C}(23)$ & $1.381(8)$ \\
\hline $\mathrm{Sr}(4)-\mathrm{O}(9)$ & $2.447(3)$ & $C(22)-C(26)$ & $1.549(8)$ \\
\hline $\operatorname{Sr}(4)-O(7)$ & $2.483(3)$ & $\mathrm{C}(23)-\mathrm{C}(24)$ & $1.384(8)$ \\
\hline $\operatorname{Sr}(4)-O(6)$ & $2.496(3)$ & $C(24)-C(27)$ & $1.500(8)$ \\
\hline $\operatorname{Sr}(4)-O(10)$ & $2.570(4)$ & $\mathrm{O}(4)-\mathrm{C}(28)$ & $1.307(6)$ \\
\hline $\operatorname{Sr}(4)-N(12)$ & $2.743(4)$ & $\mathrm{C}(28)-\mathrm{C}(29)$ & $1.408(7)$ \\
\hline $\operatorname{Sr}(4)-N(3 S)$ & $2.822(4)$ & $\mathrm{C}(28)-\mathrm{C}(33)$ & $1.422(7)$ \\
\hline $\operatorname{Sr}(4)-N(11)$ & $2.833(4)$ & $\mathrm{C}(29)-\mathrm{C}(30)$ & $1.394(7)$ \\
\hline $\mathrm{O}(1)-\mathrm{C}(1)$ & $1.333(5)$ & C(29)-C(34) & $1.518(7)$ \\
\hline $\mathrm{C}(1)-\mathrm{C}(2)$ & $1.414(6)$ & $\mathrm{C}(30)-\mathrm{C}(31)$ & $1.368(7)$ \\
\hline$C(1)-C(6)$ & $1.416(7)$ & $\mathrm{C}(31)-\mathrm{C}(32)$ & $1.380(8)$ \\
\hline $\mathrm{C}(2)-\mathrm{C}(3)$ & $1.393(7)$ & $\mathrm{C}(31)-\mathrm{C}(35)$ & $1.509(7)$ \\
\hline $\mathrm{C}(2)-\mathrm{C}(7)$ & $1.517(7)$ & $\mathrm{C}(32)-\mathrm{C}(33)$ & $1.382(7)$ \\
\hline$C(3)-C(4)$ & $1.388(7)$ & $\mathrm{C}(33)-\mathrm{C}(36)$ & $1.508(7)$ \\
\hline$C(4)-C(5)$ & $1.392(7)$ & $\mathrm{N}(1)-\mathrm{C}(37)$ & $1.457(6)$ \\
\hline $\mathrm{C}(4)-\mathrm{C}(8)$ & $1.496(7)$ & $\mathrm{N}(2)-\mathrm{C}(38)$ & $1.493(6)$ \\
\hline$C(5)-C(6)$ & $1.415(6)$ & $\mathrm{C}(37)-\mathrm{C}(38)$ & $1.512(7)$ \\
\hline$C(6)-C(9)$ & $1.489(6)$ & $\mathrm{N}(3)-\mathrm{C}(39)$ & $1.465(6)$ \\
\hline $\mathrm{O}(2)-\mathrm{C}(10)$ & $1.326(5)$ & $\mathrm{N}(4)-\mathrm{C}(40)$ & $1.477(6)$ \\
\hline
\end{tabular}




\begin{tabular}{|c|c|c|c|}
\hline$C(39)-C(40)$ & $1.517(7)$ & $C(73)-C(78)$ & $1.433(7)$ \\
\hline $\mathrm{N}(5)-\mathrm{C}(41)$ & $1.480(6)$ & $C(74)-C(75)$ & $1.384(7)$ \\
\hline $\mathrm{N}(6)-\mathrm{C}(42)$ & $1.469(6)$ & $\mathrm{C}(74)-\mathrm{C}(79)$ & $1.499(8)$ \\
\hline$C(41)-C(42)$ & $1.506(7)$ & $C(75)-C(76)$ & $1.382(8)$ \\
\hline $\mathrm{O}(6)-\mathrm{C}(46)$ & $1.340(6)$ & $C(76)-C(77)$ & $1.389(8)$ \\
\hline $\mathrm{C}(46)-\mathrm{C}(51)$ & $1.397(7)$ & $C(76)-C(80)$ & $1.530(8)$ \\
\hline$C(46)-C(47)$ & $1.414(7)$ & $C(77)-C(78)$ & $1.378(7)$ \\
\hline C(47)-C(48) & $1.367(7)$ & $\mathrm{C}(78)-\mathrm{C}(81)$ & $1.494(7)$ \\
\hline $\mathrm{C}(47)-\mathrm{C}(52)$ & $1.508(7)$ & $\mathrm{N}(7)-\mathrm{C}(82)$ & $1.471(7)$ \\
\hline $\mathrm{C}(48)-\mathrm{C}(49)$ & $1.388(8)$ & $\mathrm{N}(8)-\mathrm{C}(83)$ & $1.482(7)$ \\
\hline $\mathrm{C}(49)-\mathrm{C}(50)$ & $1.363(8)$ & $\mathrm{C}(82)-\mathrm{C}(83)$ & $1.511(7)$ \\
\hline $\mathrm{C}(49)-\mathrm{C}(53)$ & $1.529(8)$ & $\mathrm{N}(9)-\mathrm{C}(84)$ & $1.439(6)$ \\
\hline$C(50)-C(51)$ & $1.399(7)$ & $\mathrm{N}(10)-\mathrm{C}(85)$ & $1.469(7)$ \\
\hline $\mathrm{C}(51)-\mathrm{C}(54)$ & $1.501(7)$ & C(84)-C(85) & $1.482(8)$ \\
\hline $\mathrm{O}(7)-\mathrm{C}(55)$ & $1.336(6)$ & $\mathrm{N}(11)-\mathrm{C}(86)$ & $1.458(6)$ \\
\hline$C(55)-C(60)$ & $1.401(7)$ & $\mathrm{N}(12)-\mathrm{C}(87)$ & $1.466(6)$ \\
\hline$C(55)-C(56)$ & $1.410(7)$ & $\mathrm{C}(86)-\mathrm{C}(87)$ & $1.489(7)$ \\
\hline$C(56)-C(57)$ & $1.387(7)$ & $\mathrm{N}(1 \mathrm{~S})-\mathrm{C}(1 \mathrm{~S})$ & $1.470(7)$ \\
\hline$C(56)-C(61)$ & $1.517(7)$ & $\mathrm{N}(2 \mathrm{~S})-\mathrm{C}(2 \mathrm{~S})$ & $1.484(9)$ \\
\hline$C(57)-C(58)$ & $1.361(8)$ & $C(1 S)-C(2 S)$ & $1.509(8)$ \\
\hline $\mathrm{C}(58)-\mathrm{C}(59)$ & $1.393(8)$ & $\mathrm{N}(3 \mathrm{~S})-\mathrm{C}(3 \mathrm{~S})$ & $1.491(12)$ \\
\hline $\mathrm{C}(58)-\mathrm{C}(62)$ & $1.510(7)$ & $\mathrm{N}(4 \mathrm{~S})-\mathrm{C}(4 \mathrm{~S})$ & $1.503(12)$ \\
\hline$C(59)-C(60)$ & $1.401(7)$ & $C(3 S)-C(4 S)$ & $1.516(18)$ \\
\hline$C(60)-C(63)$ & $1.486(7)$ & $C\left(3 S^{\prime}\right)-C\left(4 S^{\prime}\right)$ & $1.41(3)$ \\
\hline $\mathrm{O}(8)-\mathrm{C}(64)$ & $1.333(6)$ & $\mathrm{N}(5 \mathrm{~S})-\mathrm{C}(5 \mathrm{~S})$ & $1.440(7)$ \\
\hline$C(64)-C(65)$ & $1.399(7)$ & $\mathrm{N}(6 \mathrm{~S})-\mathrm{C}(6 \mathrm{~S})$ & $1.411(7)$ \\
\hline C(64)-C(69) & $1.420(7)$ & $C(5 S)-C(6 S)$ & $1.565(10)$ \\
\hline$C(65)-C(66)$ & $1.400(7)$ & $\mathrm{N}(7 \mathrm{~S})-\mathrm{C}(7 \mathrm{~S})$ & $1.458(8)$ \\
\hline$C(65)-C(70)$ & $1.507(7)$ & $\mathrm{N}(8 \mathrm{~S})-\mathrm{C}(8 \mathrm{~S})$ & $1.432(7)$ \\
\hline$C(66)-C(67)$ & $1.379(7)$ & $\mathrm{C}(7 \mathrm{~S})-\mathrm{C}(8 \mathrm{~S})$ & $1.529(8)$ \\
\hline C(67)-C(68) & $1.390(7)$ & $\mathrm{N}(9 \mathrm{~S})-\mathrm{C}(9 \mathrm{~S})$ & $1.460(6)$ \\
\hline$C(67)-C(71)$ & $1.515(7)$ & $\mathrm{N}(10 \mathrm{~S})-\mathrm{C}(10 \mathrm{~S})$ & $1.468(6)$ \\
\hline C(68)-C(69) & $1.390(7)$ & $\mathrm{C}(9 \mathrm{~S})-\mathrm{C}(10 \mathrm{~S})$ & $1.508(7)$ \\
\hline $\mathrm{C}(69)-\mathrm{C}(72)$ & $1.512(7)$ & $\mathrm{N}(11 \mathrm{~S})-\mathrm{C}(11 \mathrm{~S})$ & $1.427(11)$ \\
\hline $\mathrm{O}(9)-\mathrm{C}(73)$ & $1.320(6)$ & $\mathrm{C}(11 \mathrm{~S})-\mathrm{C}(11 \mathrm{~S}) \# 1$ & $1.608(16)$ \\
\hline$C(73)-C(74)$ & $1.402(7)$ & & \\
\hline
\end{tabular}




\begin{tabular}{|c|c|c|c|}
\hline $\mathrm{O}(4)-\mathrm{Sr}(1)-\mathrm{O}(1)$ & $115.89(10)$ & $\mathrm{N}(2)-\mathrm{Sr}(2)-\mathrm{N}(1)$ & $64.70(12)$ \\
\hline $\mathrm{O}(4)-\operatorname{Sr}(1)-\mathrm{O}(2)$ & $167.33(11)$ & $\mathrm{O}(9)-\mathrm{Sr}(4)-\mathrm{O}(7)$ & $168.31(11)$ \\
\hline $\mathrm{O}(1)-\mathrm{Sr}(1)-\mathrm{O}(2)$ & $73.83(10)$ & $\mathrm{O}(9)-\mathrm{Sr}(4)-\mathrm{O}(6)$ & $112.32(11)$ \\
\hline $\mathrm{O}(4)-\mathrm{Sr}(1)-\mathrm{O}(5)$ & $101.92(12)$ & $\mathrm{O}(7)-\mathrm{Sr}(4)-\mathrm{O}(6)$ & $73.54(11)$ \\
\hline $\mathrm{O}(1)-\mathrm{Sr}(1)-\mathrm{O}(5)$ & $80.27(11)$ & $\mathrm{O}(9)-\mathrm{Sr}(4)-\mathrm{O}(10)$ & $104.30(12)$ \\
\hline $\mathrm{O}(2)-\mathrm{Sr}(1)-\mathrm{O}(5)$ & $87.41(11)$ & $\mathrm{O}(7)-\operatorname{Sr}(4)-\mathrm{O}(10)$ & $86.13(12)$ \\
\hline $\mathrm{O}(8)-\mathrm{Sr}(3)-\mathrm{O}(7)$ & $94.81(11)$ & $\mathrm{O}(6)-\mathrm{Sr}(4)-\mathrm{O}(10)$ & $83.85(12)$ \\
\hline $\mathrm{O}(8)-\mathrm{Sr}(3)-\mathrm{O}(6)$ & $97.09(12)$ & $\mathrm{O}(9)-\mathrm{Sr}(4)-\mathrm{N}(12)$ & $78.23(13)$ \\
\hline $\mathrm{O}(7)-\mathrm{Sr}(3)-\mathrm{O}(6)$ & $72.08(10)$ & $\mathrm{O}(7)-\operatorname{Sr}(4)-\mathrm{N}(12)$ & $100.76(12)$ \\
\hline $\mathrm{O}(8)-\mathrm{Sr}(3)-\mathrm{N}(7)$ & $89.03(12)$ & $\mathrm{O}(6)-\mathrm{Sr}(4)-\mathrm{N}(12)$ & $154.44(12)$ \\
\hline $\mathrm{O}(7)-\mathrm{Sr}(3)-\mathrm{N}(7)$ & $77.22(11)$ & $\mathrm{O}(10)-\mathrm{Sr}(4)-\mathrm{N}(12)$ & $70.81(13)$ \\
\hline $\mathrm{O}(6)-\mathrm{Sr}(3)-\mathrm{N}(7)$ & $149.07(12)$ & $\mathrm{O}(9)-\mathrm{Sr}(4)-\mathrm{N}(3 \mathrm{~S})$ & $70.30(13)$ \\
\hline $\mathrm{O}(8)-\mathrm{Sr}(3)-\mathrm{N}(10)$ & $104.55(13)$ & $\mathrm{O}(7)-\mathrm{Sr}(4)-\mathrm{N}(3 \mathrm{~S})$ & $102.55(13)$ \\
\hline $\mathrm{O}(7)-\mathrm{Sr}(3)-\mathrm{N}(10)$ & $147.38(12)$ & $\mathrm{O}(6)-\mathrm{Sr}(4)-\mathrm{N}(3 \mathrm{~S})$ & $75.70(12)$ \\
\hline $\mathrm{O}(6)-\mathrm{Sr}(3)-\mathrm{N}(10)$ & $79.58(12)$ & $\mathrm{O}(10)-\mathrm{Sr}(4)-\mathrm{N}(3 \mathrm{~S})$ & $154.25(13)$ \\
\hline $\mathrm{N}(7)-\operatorname{Sr}(3)-\mathrm{N}(10)$ & $128.20(13)$ & $\mathrm{N}(12)-\mathrm{Sr}(4)-\mathrm{N}(3 \mathrm{~S})$ & $129.53(13)$ \\
\hline $\mathrm{O}(8)-\mathrm{Sr}(3)-\mathrm{N}(9)$ & $73.25(12)$ & $\mathrm{O}(9)-\operatorname{Sr}(4)-\mathrm{N}(11)$ & $88.85(12)$ \\
\hline $\mathrm{O}(7)-\mathrm{Sr}(3)-\mathrm{N}(9)$ & $149.70(11)$ & $\mathrm{O}(7)-\operatorname{Sr}(4)-\mathrm{N}(11)$ & $80.50(12)$ \\
\hline $\mathrm{O}(6)-\mathrm{Sr}(3)-\mathrm{N}(9)$ & $135.91(11)$ & $\mathrm{O}(6)-\operatorname{Sr}(4)-\mathrm{N}(11)$ & $137.68(12)$ \\
\hline $\mathrm{N}(7)-\operatorname{Sr}(3)-\mathrm{N}(9)$ & $74.87(12)$ & $\mathrm{O}(10)-\mathrm{Sr}(4)-\mathrm{N}(11)$ & $127.51(13)$ \\
\hline $\mathrm{N}(10)-\mathrm{Sr}(3)-\mathrm{N}(9)$ & $62.58(13)$ & $\mathrm{N}(12)-\mathrm{Sr}(4)-\mathrm{N}(11)$ & $62.49(12)$ \\
\hline $\mathrm{O}(8)-\mathrm{Sr}(3)-\mathrm{N}(8)$ & $149.39(13)$ & $\mathrm{N}(3 \mathrm{~S})-\mathrm{Sr}(4)-\mathrm{N}(11)$ & $78.13(13)$ \\
\hline $\mathrm{O}(7)-\mathrm{Sr}(3)-\mathrm{N}(8)$ & $92.23(13)$ & $\mathrm{C}(1)-\mathrm{O}(1)-\operatorname{Sr}(1)$ & $122.0(3)$ \\
\hline $\mathrm{O}(6)-\mathrm{Sr}(3)-\mathrm{N}(8)$ & $113.41(13)$ & $\mathrm{C}(1)-\mathrm{O}(1)-\operatorname{Sr}(2)$ & $136.3(3)$ \\
\hline$N(7)-\operatorname{Sr}(3)-N(8)$ & $63.60(13)$ & $\mathrm{Sr}(1)-\mathrm{O}(1)-\mathrm{Sr}(2)$ & $101.41(10)$ \\
\hline $\mathrm{N}(10)-\mathrm{Sr}(3)-\mathrm{N}(8)$ & $84.35(14)$ & $\mathrm{O}(1)-\mathrm{C}(1)-\mathrm{C}(2)$ & $120.8(4)$ \\
\hline $\mathrm{N}(9)-\mathrm{Sr}(3)-\mathrm{N}(8)$ & $85.85(14)$ & $\mathrm{O}(1)-\mathrm{C}(1)-\mathrm{C}(6)$ & $120.9(4)$ \\
\hline $\mathrm{O}(3)-\mathrm{Sr}(2)-\mathrm{O}(2)$ & $99.89(11)$ & $C(2)-C(1)-C(6)$ & $118.3(4)$ \\
\hline $\mathrm{O}(3)-\mathrm{Sr}(2)-\mathrm{O}(1)$ & $94.36(12)$ & $C(3)-C(2)-C(1)$ & $120.3(5)$ \\
\hline $\mathrm{O}(2)-\mathrm{Sr}(2)-\mathrm{O}(1)$ & $73.47(10)$ & $C(3)-C(2)-C(7)$ & $120.8(4)$ \\
\hline $\mathrm{O}(3)-\mathrm{Sr}(2)-\mathrm{N}(2)$ & $154.37(13)$ & $\mathrm{C}(1)-\mathrm{C}(2)-\mathrm{C}(7)$ & $118.9(4)$ \\
\hline $\mathrm{O}(2)-\mathrm{Sr}(2)-\mathrm{N}(2)$ & $91.13(12)$ & $\mathrm{C}(4)-\mathrm{C}(3)-\mathrm{C}(2)$ & $122.3(5)$ \\
\hline $\mathrm{O}(1)-\mathrm{Sr}(2)-\mathrm{N}(2)$ & $111.00(12)$ & $C(3)-C(4)-C(5)$ & $117.5(4)$ \\
\hline $\mathrm{O}(3)-\mathrm{Sr}(2)-\mathrm{N}(1)$ & $95.84(13)$ & $\mathrm{C}(3)-\mathrm{C}(4)-\mathrm{C}(8)$ & $121.9(5)$ \\
\hline $\mathrm{O}(2)-\mathrm{Sr}(2)-\mathrm{N}(1)$ & $74.72(11)$ & $\mathrm{C}(5)-\mathrm{C}(4)-\mathrm{C}(8)$ & $120.6(5)$ \\
\hline $\mathrm{O}(1)-\mathrm{Sr}(2)-\mathrm{N}(1)$ & $147.81(11)$ & $\mathrm{C}(4)-\mathrm{C}(5)-\mathrm{C}(6)$ & $122.4(5)$ \\
\hline
\end{tabular}




\begin{tabular}{|c|c|c|c|}
\hline$C(5)-C(6)-C(1)$ & $119.0(4)$ & $\mathrm{O}(4)-\mathrm{C}(28)-\mathrm{C}(29)$ & $120.8(5)$ \\
\hline$C(5)-C(6)-C(9)$ & $120.0(4)$ & $\mathrm{O}(4)-\mathrm{C}(28)-\mathrm{C}(33)$ & $122.4(5)$ \\
\hline$C(1)-C(6)-C(9)$ & $120.9(4)$ & $\mathrm{C}(29)-\mathrm{C}(28)-\mathrm{C}(33)$ & $116.8(4)$ \\
\hline $\mathrm{C}(10)-\mathrm{O}(2)-\mathrm{Sr}(2)$ & $138.9(3)$ & $\mathrm{C}(30)-\mathrm{C}(29)-\mathrm{C}(28)$ & $120.0(5)$ \\
\hline $\mathrm{C}(10)-\mathrm{O}(2)-\mathrm{Sr}(1)$ & $118.9(3)$ & $\mathrm{C}(30)-\mathrm{C}(29)-\mathrm{C}(34)$ & $121.2(5)$ \\
\hline $\mathrm{Sr}(2)-\mathrm{O}(2)-\operatorname{Sr}(1)$ & $101.51(11)$ & $\mathrm{C}(28)-\mathrm{C}(29)-\mathrm{C}(34)$ & $118.8(4)$ \\
\hline $\mathrm{O}(2)-\mathrm{C}(10)-\mathrm{C}(15)$ & $120.9(4)$ & $C(31)-C(30)-C(29)$ & $123.1(5)$ \\
\hline $\mathrm{O}(2)-\mathrm{C}(10)-\mathrm{C}(11)$ & $121.0(5)$ & $\mathrm{C}(30)-\mathrm{C}(31)-\mathrm{C}(32)$ & $116.9(5)$ \\
\hline$C(15)-C(10)-C(11)$ & $118.1(4)$ & $\mathrm{C}(30)-\mathrm{C}(31)-\mathrm{C}(35)$ & $122.1(5)$ \\
\hline$C(12)-C(11)-C(10)$ & $119.2(5)$ & $\mathrm{C}(32)-\mathrm{C}(31)-\mathrm{C}(35)$ & $120.9(5)$ \\
\hline$C(12)-C(11)-C(16)$ & $120.3(4)$ & $\mathrm{C}(31)-\mathrm{C}(32)-\mathrm{C}(33)$ & $122.8(5)$ \\
\hline$C(10)-C(11)-C(16)$ & $120.5(4)$ & $\mathrm{C}(32)-\mathrm{C}(33)-\mathrm{C}(28)$ & $120.3(5)$ \\
\hline $\mathrm{C}(11)-\mathrm{C}(12)-\mathrm{C}(13)$ & $123.1(5)$ & $\mathrm{C}(32)-\mathrm{C}(33)-\mathrm{C}(36)$ & $120.9(5)$ \\
\hline$C(14)-C(13)-C(12)$ & $116.1(4)$ & $\mathrm{C}(28)-\mathrm{C}(33)-\mathrm{C}(36)$ & $118.7(5)$ \\
\hline$C(14)-C(13)-C(17)$ & $122.3(5)$ & $\mathrm{C}(37)-\mathrm{N}(1)-\operatorname{Sr}(2)$ & $110.4(3)$ \\
\hline$C(12)-C(13)-C(17)$ & $121.6(5)$ & $\mathrm{C}(38)-\mathrm{N}(2)-\operatorname{Sr}(2)$ & $111.2(3)$ \\
\hline$C(15)-C(14)-C(13)$ & $123.7(5)$ & $\mathrm{N}(1)-\mathrm{C}(37)-\mathrm{C}(38)$ & $111.4(4)$ \\
\hline$C(14)-C(15)-C(10)$ & $119.8(5)$ & $\mathrm{N}(2)-\mathrm{C}(38)-\mathrm{C}(37)$ & $111.6(4)$ \\
\hline$C(14)-C(15)-C(18)$ & $121.5(5)$ & $\mathrm{N}(3)-\mathrm{C}(39)-\mathrm{C}(40)$ & $109.4(4)$ \\
\hline$C(10)-C(15)-C(18)$ & $118.7(4)$ & N(4)-C(40)-C(39) & $111.3(4)$ \\
\hline $\mathrm{C}(19)-\mathrm{O}(3)-\mathrm{Sr}(2)$ & $139.4(3)$ & $\mathrm{N}(5)-\mathrm{C}(41)-\mathrm{C}(42)$ & $110.0(4)$ \\
\hline $\mathrm{O}(3)-\mathrm{C}(19)-\mathrm{C}(24)$ & $122.0(5)$ & $\mathrm{C}(46)-\mathrm{O}(6)-\mathrm{Sr}(4)$ & $128.0(3)$ \\
\hline $\mathrm{O}(3)-\mathrm{C}(19)-\mathrm{C}(20)$ & $120.3(5)$ & $\mathrm{C}(46)-\mathrm{O}(6)-\mathrm{Sr}(3)$ & $129.6(3)$ \\
\hline$C(24)-C(19)-C(20)$ & $117.7(5)$ & $\mathrm{Sr}(4)-\mathrm{O}(6)-\mathrm{Sr}(3)$ & $102.02(11)$ \\
\hline$C(21)-C(20)-C(19)$ & $119.9(5)$ & $\mathrm{N}(6)-\mathrm{C}(42)-\mathrm{C}(41)$ & $109.1(4)$ \\
\hline$C(21)-C(20)-C(25)$ & $121.0(5)$ & $\mathrm{O}(6)-\mathrm{C}(46)-\mathrm{C}(51)$ & $120.3(5)$ \\
\hline$C(19)-C(20)-C(25)$ & 119.1(5) & $\mathrm{O}(6)-\mathrm{C}(46)-\mathrm{C}(47)$ & $121.0(5)$ \\
\hline$C(22)-C(21)-C(20)$ & $122.2(6)$ & $C(51)-C(46)-C(47)$ & $118.7(5)$ \\
\hline $\mathrm{C}(21)-\mathrm{C}(22)-\mathrm{C}(23)$ & $118.7(5)$ & $C(48)-C(47)-C(46)$ & $119.0(5)$ \\
\hline $\mathrm{C}(21)-\mathrm{C}(22)-\mathrm{C}(26)$ & $121.8(6)$ & $\mathrm{C}(48)-\mathrm{C}(47)-\mathrm{C}(52)$ & $121.2(5)$ \\
\hline $\mathrm{C}(23)-\mathrm{C}(22)-\mathrm{C}(26)$ & $119.5(6)$ & $C(46)-C(47)-C(52)$ & $119.8(4)$ \\
\hline $\mathrm{C}(22)-\mathrm{C}(23)-\mathrm{C}(24)$ & $121.6(6)$ & $\mathrm{C}(47)-\mathrm{C}(48)-\mathrm{C}(49)$ & $123.4(5)$ \\
\hline $\mathrm{C}(23)-\mathrm{C}(24)-\mathrm{C}(19)$ & $119.9(5)$ & $C(50)-C(49)-C(48)$ & $116.8(5)$ \\
\hline$C(23)-C(24)-C(27)$ & $122.1(5)$ & $C(50)-C(49)-C(53)$ & $123.1(6)$ \\
\hline$C(19)-C(24)-C(27)$ & $118.0(5)$ & $\mathrm{C}(48)-\mathrm{C}(49)-\mathrm{C}(53)$ & $120.1(6)$ \\
\hline $\mathrm{C}(28)-\mathrm{O}(4)-\mathrm{Sr}(1)$ & $147.4(3)$ & $C(49)-C(50)-C(51)$ & $122.8(5)$ \\
\hline
\end{tabular}




\begin{tabular}{|c|c|c|c|}
\hline$C(46)-C(51)-C(50)$ & 119.1(5) & $\mathrm{O}(9)-\mathrm{C}(73)-\mathrm{C}(74)$ & $120.5(5)$ \\
\hline$C(46)-C(51)-C(54)$ & $120.4(5)$ & $\mathrm{O}(9)-\mathrm{C}(73)-\mathrm{C}(78)$ & $121.1(5)$ \\
\hline $\mathrm{C}(50)-\mathrm{C}(51)-\mathrm{C}(54)$ & $120.6(5)$ & $\mathrm{C}(74)-\mathrm{C}(73)-\mathrm{C}(78)$ & $118.4(5)$ \\
\hline $\mathrm{C}(55)-\mathrm{O}(7)-\mathrm{Sr}(4)$ & $124.4(3)$ & $C(75)-C(74)-C(73)$ & $119.7(5)$ \\
\hline $\mathrm{C}(55)-\mathrm{O}(7)-\mathrm{Sr}(3)$ & $131.7(3)$ & $\mathrm{C}(75)-\mathrm{C}(74)-\mathrm{C}(79)$ & $121.8(5)$ \\
\hline $\mathrm{Sr}(4)-\mathrm{O}(7)-\mathrm{Sr}(3)$ & $103.14(11)$ & $\mathrm{C}(73)-\mathrm{C}(74)-\mathrm{C}(79)$ & $118.6(5)$ \\
\hline $\mathrm{O}(7)-\mathrm{C}(55)-\mathrm{C}(60)$ & $120.6(4)$ & $C(76)-C(75)-C(74)$ & $123.4(5)$ \\
\hline $\mathrm{O}(7)-\mathrm{C}(55)-\mathrm{C}(56)$ & $120.4(5)$ & $C(75)-C(76)-C(77)$ & $116.1(5)$ \\
\hline $\mathrm{C}(60)-\mathrm{C}(55)-\mathrm{C}(56)$ & $118.9(4)$ & $C(75)-C(76)-C(80)$ & $121.1(6)$ \\
\hline $\mathrm{C}(57)-\mathrm{C}(56)-\mathrm{C}(55)$ & $118.7(5)$ & $\mathrm{C}(77)-\mathrm{C}(76)-\mathrm{C}(80)$ & $122.7(6)$ \\
\hline $\mathrm{C}(57)-\mathrm{C}(56)-\mathrm{C}(61)$ & $120.8(5)$ & $\mathrm{C}(78)-\mathrm{C}(77)-\mathrm{C}(76)$ & $124.0(5)$ \\
\hline $\mathrm{C}(55)-\mathrm{C}(56)-\mathrm{C}(61)$ & $120.5(4)$ & $\mathrm{C}(77)-\mathrm{C}(78)-\mathrm{C}(73)$ & $118.4(5)$ \\
\hline $\mathrm{C}(58)-\mathrm{C}(57)-\mathrm{C}(56)$ & $123.9(5)$ & $\mathrm{C}(77)-\mathrm{C}(78)-\mathrm{C}(81)$ & $121.9(5)$ \\
\hline $\mathrm{C}(57)-\mathrm{C}(58)-\mathrm{C}(59)$ & $117.1(5)$ & $\mathrm{C}(73)-\mathrm{C}(78)-\mathrm{C}(81)$ & $119.7(5)$ \\
\hline $\mathrm{C}(57)-\mathrm{C}(58)-\mathrm{C}(62)$ & $122.9(6)$ & $\mathrm{C}(82)-\mathrm{N}(7)-\operatorname{Sr}(3)$ & $113.3(3)$ \\
\hline $\mathrm{C}(59)-\mathrm{C}(58)-\mathrm{C}(62)$ & $119.9(6)$ & $\mathrm{C}(83)-\mathrm{N}(8)-\operatorname{Sr}(3)$ & $111.4(3)$ \\
\hline $\mathrm{C}(58)-\mathrm{C}(59)-\mathrm{C}(60)$ & $121.9(5)$ & $\mathrm{N}(7)-\mathrm{C}(82)-\mathrm{C}(83)$ & $110.1(4)$ \\
\hline$C(55)-C(60)-C(59)$ & $119.4(5)$ & $\mathrm{N}(8)-\mathrm{C}(83)-\mathrm{C}(82)$ & $110.6(4)$ \\
\hline $\mathrm{C}(55)-\mathrm{C}(60)-\mathrm{C}(63)$ & $120.9(4)$ & $\mathrm{C}(84)-\mathrm{N}(9)-\mathrm{Sr}(3)$ & $116.1(3)$ \\
\hline$C(59)-C(60)-C(63)$ & $119.7(5)$ & $\mathrm{C}(85)-\mathrm{N}(10)-\mathrm{Sr}(3)$ & $111.2(3)$ \\
\hline $\mathrm{C}(64)-\mathrm{O}(8)-\mathrm{Sr}(3)$ & $151.7(3)$ & $\mathrm{N}(9)-\mathrm{C}(84)-\mathrm{C}(85)$ & $112.0(4)$ \\
\hline $\mathrm{O}(8)-\mathrm{C}(64)-\mathrm{C}(65)$ & $121.4(5)$ & $\mathrm{N}(10)-\mathrm{C}(85)-\mathrm{C}(84)$ & $112.2(4)$ \\
\hline $\mathrm{O}(8)-\mathrm{C}(64)-\mathrm{C}(69)$ & $120.7(4)$ & $\mathrm{C}(86)-\mathrm{N}(11)-\mathrm{Sr}(4)$ & $112.1(3)$ \\
\hline$C(65)-C(64)-C(69)$ & $117.8(5)$ & $\mathrm{C}(87)-\mathrm{N}(12)-\mathrm{Sr}(4)$ & $115.1(3)$ \\
\hline$C(64)-C(65)-C(66)$ & $120.8(5)$ & $\mathrm{N}(11)-\mathrm{C}(86)-\mathrm{C}(87)$ & $110.6(4)$ \\
\hline$C(64)-C(65)-C(70)$ & $119.5(5)$ & $\mathrm{N}(12)-\mathrm{C}(87)-\mathrm{C}(86)$ & $110.7(4)$ \\
\hline$C(66)-C(65)-C(70)$ & $119.7(5)$ & $\mathrm{N}(1 \mathrm{~S})-\mathrm{C}(1 \mathrm{~S})-\mathrm{C}(2 \mathrm{~S})$ & $114.4(5)$ \\
\hline$C(67)-C(66)-C(65)$ & $121.6(5)$ & $\mathrm{N}(2 \mathrm{~S})-\mathrm{C}(2 \mathrm{~S})-\mathrm{C}(1 \mathrm{~S})$ & $114.3(6)$ \\
\hline$C(66)-C(67)-C(68)$ & $117.6(5)$ & $\mathrm{C}(3 \mathrm{~S})-\mathrm{N}(3 \mathrm{~S})-\mathrm{Sr}(4)$ & $130.5(5)$ \\
\hline$C(66)-C(67)-C(71)$ & $121.4(5)$ & $\mathrm{N}(3 \mathrm{~S})-\mathrm{C}(3 \mathrm{~S})-\mathrm{C}(4 \mathrm{~S})$ & $109.6(9)$ \\
\hline$C(68)-C(67)-C(71)$ & $121.0(5)$ & $\mathrm{N}(4 \mathrm{~S})-\mathrm{C}(4 \mathrm{~S})-\mathrm{C}(3 \mathrm{~S})$ & $107.3(9)$ \\
\hline$C(67)-C(68)-C(69)$ & $122.8(5)$ & $\mathrm{N}(5 \mathrm{~S})-\mathrm{C}(5 \mathrm{~S})-\mathrm{C}(6 \mathrm{~S})$ & $113.3(6)$ \\
\hline $\mathrm{C}(68)-\mathrm{C}(69)-\mathrm{C}(64)$ & $119.3(5)$ & $\mathrm{N}(6 \mathrm{~S})-\mathrm{C}(6 \mathrm{~S})-\mathrm{C}(5 \mathrm{~S})$ & $112.5(6)$ \\
\hline $\mathrm{C}(68)-\mathrm{C}(69)-\mathrm{C}(72)$ & $120.5(5)$ & $\mathrm{N}(7 \mathrm{~S})-\mathrm{C}(7 \mathrm{~S})-\mathrm{C}(8 \mathrm{~S})$ & $113.4(6)$ \\
\hline$C(64)-C(69)-C(72)$ & $120.2(5)$ & $\mathrm{N}(8 \mathrm{~S})-\mathrm{C}(8 \mathrm{~S})-\mathrm{C}(7 \mathrm{~S})$ & $114.7(5)$ \\
\hline $\mathrm{C}(73)-\mathrm{O}(9)-\mathrm{Sr}(4)$ & $137.4(3)$ & N(9S)-C(9S)-C(10S) & $113.9(4)$ \\
\hline
\end{tabular}


Symmetry transformations used to generate equivalent atoms:

\#1 -x,-y,-z+2 
Table 4. Anisotropic displacement parameters $\left(\AA^{2} \times 10^{3}\right)$ for compound 5. The anisotropic displacement factor exponent takes the form: $-2 \pi^{2}\left[h^{2} a^{* 2} U^{11}+\ldots+2 h k^{*} b^{*} U^{12}\right]$

\begin{tabular}{|c|c|c|c|c|c|c|}
\hline & $\mathrm{U}^{11}$ & $\mathrm{U}^{22}$ & $\mathrm{U}^{33}$ & $\mathrm{U}^{23}$ & $\mathrm{U}^{13}$ & $\mathrm{U}^{12}$ \\
\hline $\operatorname{Sr}(1)$ & $16(1)$ & $18(1)$ & $16(1)$ & $2(1)$ & $6(1)$ & $-1(1)$ \\
\hline $\operatorname{Sr}(3)$ & $18(1)$ & 19(1) & $18(1)$ & $-1(1)$ & $5(1)$ & $-1(1)$ \\
\hline $\operatorname{Sr}(2)$ & $18(1)$ & $18(1)$ & $15(1)$ & $0(1)$ & $5(1)$ & $-2(1)$ \\
\hline $\operatorname{Sr}(4)$ & $19(1)$ & $23(1)$ & $16(1)$ & $1(1)$ & $5(1)$ & $-2(1)$ \\
\hline $\mathrm{O}(1)$ & $19(2)$ & $21(2)$ & $14(2)$ & $-3(2)$ & $5(2)$ & $-7(2)$ \\
\hline $\mathrm{C}(1)$ & $9(2)$ & $26(3)$ & $15(3)$ & $-1(2)$ & $3(2)$ & $-5(2)$ \\
\hline$C(2)$ & 11(3) & $24(3)$ & $13(3)$ & $4(2)$ & $-2(2)$ & $-2(2)$ \\
\hline$C(3)$ & $35(3)$ & $23(3)$ & $22(3)$ & $7(2)$ & $17(3)$ & $3(2)$ \\
\hline$C(4)$ & $36(3)$ & $33(4)$ & $24(3)$ & $-4(3)$ & $13(3)$ & 4(3) \\
\hline$C(5)$ & $24(3)$ & $17(3)$ & $18(3)$ & $0(2)$ & $4(2)$ & $5(2)$ \\
\hline$C(6)$ & $13(2)$ & 19(3) & $12(3)$ & $3(2)$ & $1(2)$ & $1(2)$ \\
\hline$C(7)$ & $40(3)$ & $23(3)$ & $19(3)$ & $5(2)$ & $11(3)$ & 2(3) \\
\hline$C(8)$ & $54(4)$ & $37(4)$ & $24(3)$ & $5(3)$ & $11(3)$ & 19(3) \\
\hline$C(9)$ & $40(3)$ & $25(3)$ & $25(3)$ & $3(2)$ & $13(3)$ & $-1(3)$ \\
\hline $\mathrm{O}(2)$ & $19(2)$ & $23(2)$ & $21(2)$ & $-2(2)$ & $11(2)$ & $-3(2)$ \\
\hline$C(10)$ & $19(3)$ & $20(3)$ & $19(3)$ & $-7(2)$ & $6(2)$ & $-7(2)$ \\
\hline $\mathrm{C}(11)$ & $24(3)$ & 21(3) & $21(3)$ & $0(2)$ & $5(2)$ & $-3(2)$ \\
\hline$C(12)$ & $41(3)$ & $18(3)$ & $29(3)$ & $-4(2)$ & $23(3)$ & $-10(3)$ \\
\hline$C(13)$ & $35(3)$ & $15(3)$ & $44(4)$ & $-8(3)$ & $27(3)$ & $-4(2)$ \\
\hline$C(14)$ & $25(3)$ & $15(3)$ & $34(3)$ & $8(2)$ & $10(3)$ & $3(2)$ \\
\hline$C(15)$ & $22(3)$ & $16(3)$ & $26(3)$ & $4(2)$ & $8(2)$ & $-1(2)$ \\
\hline$C(16)$ & $34(3)$ & $24(3)$ & $29(3)$ & $-3(3)$ & $15(3)$ & $-1(3)$ \\
\hline$C(17)$ & $51(4)$ & $30(4)$ & $57(4)$ & $4(3)$ & $35(4)$ & $7(3)$ \\
\hline$C(18)$ & $32(3)$ & $27(3)$ & $29(3)$ & $0(3)$ & $7(3)$ & $-3(3)$ \\
\hline $\mathrm{O}(3)$ & $18(2)$ & $33(2)$ & $27(2)$ & $10(2)$ & $0(2)$ & $-2(2)$ \\
\hline C(19) & $20(3)$ & $32(3)$ & $26(3)$ & $16(3)$ & $4(3)$ & $-5(2)$ \\
\hline$C(20)$ & $29(3)$ & $21(3)$ & $31(3)$ & $14(3)$ & $5(3)$ & $-6(2)$ \\
\hline $\mathrm{C}(21)$ & $31(3)$ & 31(4) & $34(3)$ & 11(3) & $-2(3)$ & $-12(3)$ \\
\hline$C(22)$ & $23(3)$ & 41(4) & $45(4)$ & $26(3)$ & $-4(3)$ & $-8(3)$ \\
\hline$C(23)$ & $37(4)$ & $22(3)$ & $54(4)$ & $18(3)$ & $24(3)$ & $7(3)$ \\
\hline$C(24)$ & $24(3)$ & 27(3) & $38(4)$ & $12(3)$ & $10(3)$ & $-4(3)$ \\
\hline
\end{tabular}




\begin{tabular}{|c|c|c|c|c|c|c|}
\hline$C(25)$ & $49(4)$ & $32(4)$ & $30(3)$ & $9(3)$ & $12(3)$ & $0(3)$ \\
\hline$C(26)$ & $31(4)$ & $70(6)$ & $74(5)$ & $26(4)$ & $-9(4)$ & $-11(4)$ \\
\hline$C(27)$ & $37(4)$ & $38(4)$ & $42(4)$ & $17(3)$ & $14(3)$ & $6(3)$ \\
\hline $\mathrm{O}(4)$ & $26(2)$ & $18(2)$ & $35(2)$ & $1(2)$ & $13(2)$ & $-4(2)$ \\
\hline$C(28)$ & $30(3)$ & $18(3)$ & $22(3)$ & $-9(2)$ & $16(3)$ & $-6(2)$ \\
\hline$C(29)$ & $27(3)$ & $17(3)$ & $21(3)$ & $-6(2)$ & $15(3)$ & $-1(2)$ \\
\hline$C(30)$ & $26(3)$ & $30(3)$ & $28(3)$ & $-15(3)$ & $17(3)$ & $-8(3)$ \\
\hline$C(31)$ & $40(4)$ & 21(3) & $29(3)$ & $-5(3)$ & $25(3)$ & $-6(3)$ \\
\hline$C(32)$ & 41(4) & $19(3)$ & $19(3)$ & $2(2)$ & $14(3)$ & $8(3)$ \\
\hline$C(33)$ & $18(3)$ & $26(3)$ & $18(3)$ & $-11(2)$ & $3(2)$ & $0(2)$ \\
\hline$C(34)$ & $28(3)$ & $25(3)$ & $27(3)$ & $-1(3)$ & $11(3)$ & $-3(2)$ \\
\hline$C(35)$ & $55(4)$ & $31(4)$ & $42(4)$ & $-10(3)$ & $32(3)$ & $-15(3)$ \\
\hline $\mathrm{O}(5)$ & $23(2)$ & $24(2)$ & $23(2)$ & $2(2)$ & $6(2)$ & $-7(2)$ \\
\hline $\mathrm{N}(1)$ & $35(3)$ & 21(3) & $24(2)$ & $-4(2)$ & $17(2)$ & $0(2)$ \\
\hline $\mathrm{N}(2)$ & $50(3)$ & $19(3)$ & $21(2)$ & $7(2)$ & $15(2)$ & $-10(2)$ \\
\hline$C(36)$ & $23(3)$ & $30(3)$ & $26(3)$ & $10(3)$ & $-2(3)$ & $7(2)$ \\
\hline$C(37)$ & $36(3)$ & $16(3)$ & $41(4)$ & $0(3)$ & $15(3)$ & $5(3)$ \\
\hline $\mathrm{N}(3)$ & $23(2)$ & $32(3)$ & $19(2)$ & $-9(2)$ & $7(2)$ & $-18(2)$ \\
\hline $\mathrm{N}(4)$ & $21(2)$ & $43(3)$ & $27(3)$ & $-18(2)$ & $11(2)$ & $-7(2)$ \\
\hline$C(38)$ & $38(3)$ & $17(3)$ & $42(4)$ & $4(3)$ & $13(3)$ & $-3(3)$ \\
\hline C(39) & $23(3)$ & $27(3)$ & $26(3)$ & $3(3)$ & $10(3)$ & $-3(2)$ \\
\hline $\mathrm{N}(5)$ & $14(2)$ & $36(3)$ & $30(3)$ & $5(2)$ & $8(2)$ & $-6(2)$ \\
\hline $\mathrm{N}(6)$ & $17(2)$ & $50(3)$ & $10(2)$ & $7(2)$ & $4(2)$ & $7(2)$ \\
\hline $\mathrm{C}(40)$ & $18(3)$ & $37(3)$ & $24(3)$ & $2(3)$ & $6(2)$ & $-4(2)$ \\
\hline $\mathrm{C}(41)$ & $31(3)$ & $22(3)$ & $31(3)$ & $4(3)$ & $17(3)$ & $0(2)$ \\
\hline $\mathrm{O}(6)$ & $22(2)$ & $23(2)$ & $29(2)$ & $3(2)$ & $10(2)$ & $1(2)$ \\
\hline$C(42)$ & $35(3)$ & $20(3)$ & $34(3)$ & $1(3)$ & $26(3)$ & $-3(2)$ \\
\hline$C(46)$ & $25(3)$ & $32(3)$ & $14(3)$ & $6(2)$ & $4(2)$ & $2(2)$ \\
\hline$C(47)$ & $20(3)$ & $27(3)$ & $21(3)$ & $3(2)$ & $1(2)$ & $1(2)$ \\
\hline C(48) & $41(4)$ & $35(4)$ & $25(3)$ & $-3(3)$ & $8(3)$ & $12(3)$ \\
\hline C(49) & $33(4)$ & $42(4)$ & $47(4)$ & $0(3)$ & $18(3)$ & $7(3)$ \\
\hline$C(50)$ & $17(3)$ & $37(4)$ & $43(4)$ & $7(3)$ & $5(3)$ & $-7(3)$ \\
\hline$C(51)$ & $22(3)$ & $27(3)$ & $23(3)$ & $6(2)$ & $1(2)$ & $-3(2)$ \\
\hline$C(52)$ & $29(3)$ & $33(3)$ & $37(4)$ & $1(3)$ & $6(3)$ & $2(3)$ \\
\hline$C(53)$ & $51(5)$ & $80(6)$ & $76(5)$ & $-25(5)$ & $32(4)$ & $4(4)$ \\
\hline $\mathrm{O}(7)$ & $18(2)$ & $26(2)$ & $20(2)$ & $-4(2)$ & $4(2)$ & $3(2)$ \\
\hline
\end{tabular}




\begin{tabular}{|c|c|c|c|c|c|c|}
\hline$C(54)$ & $33(3)$ & $33(4)$ & $41(4)$ & 1(3) & $12(3)$ & $-3(3)$ \\
\hline $\mathrm{C}(55)$ & $29(3)$ & $20(3)$ & $17(3)$ & $4(2)$ & $8(2)$ & $-4(2)$ \\
\hline$C(56)$ & $17(3)$ & $29(3)$ & $23(3)$ & $0(2)$ & $9(2)$ & $1(2)$ \\
\hline$C(57)$ & $28(3)$ & $37(4)$ & $35(3)$ & $0(3)$ & $18(3)$ & $2(3)$ \\
\hline$C(58)$ & $46(4)$ & $38(4)$ & $38(4)$ & $-6(3)$ & $24(3)$ & $3(3)$ \\
\hline $\mathrm{C}(59)$ & $40(4)$ & $30(3)$ & $8(3)$ & $-2(2)$ & $4(3)$ & $-6(3)$ \\
\hline $\mathrm{C}(60)$ & $23(3)$ & $24(3)$ & $12(3)$ & $3(2)$ & $0(2)$ & $-3(2)$ \\
\hline$C(61)$ & $26(3)$ & $40(4)$ & $27(3)$ & $-5(3)$ & $8(3)$ & $-5(3)$ \\
\hline$C(62)$ & $61(5)$ & $59(5)$ & $42(4)$ & $-12(3)$ & $25(4)$ & $6(4)$ \\
\hline $\mathrm{O}(8)$ & $26(2)$ & $27(2)$ & $21(2)$ & $-4(2)$ & $3(2)$ & $-8(2)$ \\
\hline$C(63)$ & $33(3)$ & $33(3)$ & $30(3)$ & $-2(3)$ & $9(3)$ & $-5(3)$ \\
\hline$C(64)$ & $27(3)$ & $20(3)$ & $14(3)$ & $2(2)$ & $7(2)$ & $-6(2)$ \\
\hline$C(65)$ & $22(3)$ & $27(3)$ & $17(3)$ & $4(2)$ & $6(2)$ & $-4(2)$ \\
\hline $\mathrm{C}(66)$ & $29(3)$ & $24(3)$ & $15(3)$ & $-8(2)$ & $8(2)$ & $-7(2)$ \\
\hline$C(67)$ & $23(3)$ & $36(3)$ & $18(3)$ & $-1(3)$ & $8(2)$ & $-9(3)$ \\
\hline$C(68)$ & $22(3)$ & $28(3)$ & $23(3)$ & $4(2)$ & $9(3)$ & $-3(2)$ \\
\hline $\mathrm{C}(69)$ & 21(3) & $27(3)$ & $20(3)$ & $1(2)$ & $7(2)$ & $-10(2)$ \\
\hline$C(70)$ & $31(3)$ & $28(3)$ & $23(3)$ & $-2(2)$ & $7(3)$ & $-4(3)$ \\
\hline$C(71)$ & $33(3)$ & $61(4)$ & $21(3)$ & $-6(3)$ & $2(3)$ & $-12(3)$ \\
\hline $\mathrm{O}(9)$ & $29(2)$ & $23(2)$ & $25(2)$ & $6(2)$ & $7(2)$ & $-3(2)$ \\
\hline $\mathrm{C}(72)$ & $30(3)$ & $30(3)$ & $26(3)$ & $3(3)$ & $6(3)$ & $0(3)$ \\
\hline$C(73)$ & $30(3)$ & $24(3)$ & $28(3)$ & $5(3)$ & $15(3)$ & $7(3)$ \\
\hline$C(74)$ & $20(3)$ & $30(3)$ & $26(3)$ & $5(3)$ & $10(3)$ & $10(2)$ \\
\hline$C(75)$ & $37(4)$ & $39(4)$ & $27(3)$ & $13(3)$ & $18(3)$ & $13(3)$ \\
\hline$C(76)$ & $32(3)$ & $29(3)$ & $49(4)$ & $8(3)$ & $23(3)$ & $2(3)$ \\
\hline$C(77)$ & $29(3)$ & $21(3)$ & $50(4)$ & $5(3)$ & $21(3)$ & $2(3)$ \\
\hline$C(78)$ & $22(3)$ & $27(3)$ & $28(3)$ & $5(3)$ & $6(3)$ & $6(2)$ \\
\hline$C(79)$ & $43(4)$ & $37(4)$ & $20(3)$ & $5(3)$ & $9(3)$ & $5(3)$ \\
\hline$C(80)$ & $59(4)$ & 41(4) & $61(5)$ & $17(3)$ & $34(4)$ & $-5(3)$ \\
\hline $\mathrm{C}(81)$ & $36(3)$ & $31(3)$ & $26(3)$ & $4(3)$ & $5(3)$ & $-2(3)$ \\
\hline $\mathrm{O}(10)$ & $38(3)$ & $23(2)$ & $18(2)$ & $3(2)$ & $-2(2)$ & $-8(2)$ \\
\hline $\mathrm{N}(7)$ & $31(3)$ & $16(2)$ & $41(3)$ & $-10(2)$ & $24(2)$ & $-6(2)$ \\
\hline N(8) & $37(3)$ & $21(3)$ & $59(3)$ & $-2(2)$ & $20(3)$ & $-12(2)$ \\
\hline $\mathrm{C}(82)$ & $46(4)$ & $25(3)$ & $28(3)$ & $-2(3)$ & $14(3)$ & $4(3)$ \\
\hline $\mathrm{C}(83)$ & $35(3)$ & $17(3)$ & $33(3)$ & $2(3)$ & $2(3)$ & $-10(3)$ \\
\hline N(9) & $31(3)$ & $29(3)$ & $20(2)$ & $17(2)$ & $7(2)$ & $9(2)$ \\
\hline
\end{tabular}




\begin{tabular}{|c|c|c|c|c|c|c|}
\hline $\mathrm{N}(10)$ & $40(3)$ & $45(3)$ & $32(3)$ & $7(2)$ & $24(3)$ & $21(2)$ \\
\hline$C(84)$ & $36(3)$ & $35(4)$ & $18(3)$ & $9(3)$ & $5(3)$ & $-4(3)$ \\
\hline $\mathrm{C}(85)$ & $50(4)$ & $46(4)$ & $31(4)$ & $-9(3)$ & $25(3)$ & $-9(3)$ \\
\hline $\mathrm{N}(11)$ & $38(3)$ & $41(3)$ & $12(2)$ & $4(2)$ & $6(2)$ & $-8(2)$ \\
\hline $\mathrm{N}(12)$ & $18(2)$ & $64(4)$ & $22(3)$ & $-14(2)$ & $9(2)$ & $-7(2)$ \\
\hline$C(86)$ & $34(3)$ & $41(4)$ & $24(3)$ & $-4(3)$ & $10(3)$ & $-9(3)$ \\
\hline C(87) & $23(3)$ & $68(5)$ & $22(3)$ & $-8(3)$ & $7(3)$ & $-3(3)$ \\
\hline $\mathrm{N}(1 \mathrm{~S})$ & $27(3)$ & $21(3)$ & $24(2)$ & $-9(2)$ & $1(2)$ & $-4(2)$ \\
\hline $\mathrm{N}(2 \mathrm{~S})$ & $57(4)$ & $84(5)$ & $57(4)$ & $24(4)$ & $-25(3)$ & $-25(4)$ \\
\hline $\mathrm{C}(1 \mathrm{~S})$ & $30(3)$ & $39(4)$ & $25(3)$ & $3(3)$ & $4(3)$ & $-1(3)$ \\
\hline $\mathrm{C}(2 \mathrm{~S})$ & $73(5)$ & $54(5)$ & 29(4) & $14(3)$ & $-7(4)$ & $-12(4)$ \\
\hline $\mathrm{N}(3 \mathrm{~S})$ & $23(3)$ & $48(3)$ & $24(3)$ & $-1(2)$ & $-6(2)$ & $-8(2)$ \\
\hline $\mathrm{N}(4 \mathrm{~S})$ & $50(4)$ & $79(5)$ & $47(4)$ & $-8(3)$ & $-12(3)$ & $-23(3)$ \\
\hline $\mathrm{C}(3 \mathrm{~S})$ & $15(6)$ & $36(6)$ & $28(8)$ & $3(5)$ & $1(6)$ & $4(5)$ \\
\hline $\mathrm{C}(4 \mathrm{~S})$ & $20(7)$ & $41(7)$ & $47(8)$ & $-5(5)$ & $-10(6)$ & $16(5)$ \\
\hline $\mathrm{N}\left(3 \mathrm{~S}^{\prime}\right)$ & $23(3)$ & $48(3)$ & $24(3)$ & $-1(2)$ & $-6(2)$ & $-8(2)$ \\
\hline $\mathrm{N}\left(4 \mathrm{~S}^{\prime}\right)$ & $50(4)$ & $79(5)$ & $47(4)$ & $-8(3)$ & $-12(3)$ & $-23(3)$ \\
\hline$C\left(3 S^{\prime}\right)$ & $19(12)$ & $52(12)$ & $56(15)$ & $20(10)$ & $10(11)$ & $4(9)$ \\
\hline$C\left(4 S^{\prime}\right)$ & $28(12)$ & $57(13)$ & $34(13)$ & $9(10)$ & $-15(10)$ & $-22(10)$ \\
\hline $\mathrm{N}(5 \mathrm{~S})$ & $77(4)$ & $36(3)$ & $35(3)$ & $4(3)$ & $5(3)$ & $39(3)$ \\
\hline $\mathrm{N}(6 \mathrm{~S})$ & $32(3)$ & $45(3)$ & $63(4)$ & $28(3)$ & $22(3)$ & $18(2)$ \\
\hline $\mathrm{C}(5 \mathrm{~S})$ & $144(8)$ & $46(5)$ & $25(4)$ & $9(3)$ & $18(5)$ & $43(5)$ \\
\hline $\mathrm{C}(6 \mathrm{~S})$ & $110(7)$ & $48(5)$ & $69(5)$ & $21(4)$ & $61(5)$ & $35(5)$ \\
\hline $\mathrm{N}(7 \mathrm{~S})$ & $97(5)$ & $58(4)$ & $81(5)$ & $-5(3)$ & $61(4)$ & $23(4)$ \\
\hline $\mathrm{N}(8 \mathrm{~S})$ & 64(4) & $55(4)$ & $88(5)$ & $-41(3)$ & $50(4)$ & $-28(3)$ \\
\hline $\mathrm{C}(7 \mathrm{~S})$ & $58(5)$ & $63(5)$ & $47(4)$ & $-14(4)$ & $25(4)$ & $-1(4)$ \\
\hline $\mathrm{C}(8 \mathrm{~S})$ & 41(4) & $42(4)$ & $40(4)$ & $3(3)$ & $15(3)$ & 11(3) \\
\hline $\mathrm{N}(9 \mathrm{~S})$ & $22(2)$ & $31(3)$ & $32(3)$ & $-12(2)$ & $-7(2)$ & $-7(2)$ \\
\hline $\mathrm{N}(10 \mathrm{~S})$ & $28(3)$ & $50(3)$ & $26(3)$ & $-28(2)$ & $-3(2)$ & $0(2)$ \\
\hline $\mathrm{C}(9 \mathrm{~S})$ & $27(3)$ & $17(3)$ & $20(3)$ & $-4(2)$ & $8(2)$ & $-5(2)$ \\
\hline $\mathrm{C}(10 \mathrm{~S})$ & $24(3)$ & $25(3)$ & $17(3)$ & $-3(2)$ & $5(2)$ & $6(2)$ \\
\hline $\mathrm{N}(11 \mathrm{~S})$ & $157(9)$ & $35(5)$ & $183(10)$ & $-1(5)$ & $3(8)$ & $-20(5)$ \\
\hline $\mathrm{C}(11 \mathrm{~S})$ & 131(9) & $54(6)$ & $75(6)$ & $-9(5)$ & $14(6)$ & $-20(6)$ \\
\hline
\end{tabular}


Table 5. Hydrogen coordinates $\left(\times 10^{4}\right)$ and isotropic displacement parameters $\left(\AA^{2} \times 10^{3}\right)$ for compound 5.

\begin{tabular}{|c|c|c|c|c|}
\hline & $\mathrm{x}$ & $\mathrm{y}$ & $\mathrm{z}$ & $\mathrm{U}(\mathrm{eq})$ \\
\hline $\mathrm{H}(3 \mathrm{~B})$ & 5224 & 1931 & 5994 & 30 \\
\hline $\mathrm{H}(5 \mathrm{C})$ & 5653 & 98 & 6781 & 25 \\
\hline $\mathrm{H}(7 \mathrm{~A})$ & 5042 & 2889 & 6469 & 41 \\
\hline $\mathrm{H}(7 \mathrm{~B})$ & 5428 & 2935 & 7147 & 41 \\
\hline $\mathrm{H}(7 \mathrm{C})$ & 4784 & 2698 & 6949 & 41 \\
\hline $\mathrm{H}(8 \mathrm{~A})$ & 5592 & 184 & 5823 & 59 \\
\hline $\mathrm{H}(8 \mathrm{~B})$ & 5773 & 915 & 5656 & 59 \\
\hline $\mathrm{H}(8 \mathrm{C})$ & 5120 & 711 & 5437 & 59 \\
\hline $\mathrm{H}(9 \mathrm{~A})$ & 5666 & 653 & 8171 & 44 \\
\hline $\mathrm{H}(9 \mathrm{~B})$ & 6059 & 183 & 7950 & 44 \\
\hline $\mathrm{H}(9 \mathrm{C})$ & 5400 & 20 & 7746 & 44 \\
\hline $\mathrm{H}(12 \mathrm{~A})$ & 4478 & 3320 & 10021 & 32 \\
\hline $\mathrm{H}(14 \mathrm{~A})$ & 3602 & 4052 & 8419 & 30 \\
\hline $\mathrm{H}(16 \mathrm{~A})$ & 5459 & 2354 & 9610 & 42 \\
\hline $\mathrm{H}(16 \mathrm{~B})$ & 5090 & 2214 & 9998 & 42 \\
\hline $\mathrm{H}(16 \mathrm{C})$ & 5504 & 2860 & 10135 & 42 \\
\hline $\mathrm{H}(17 \mathrm{~A})$ & 3723 & 4065 & 9921 & 63 \\
\hline $\mathrm{H}(17 \mathrm{~B})$ & 3209 & 3998 & 9307 & 63 \\
\hline $\mathrm{H}(17 \mathrm{C})$ & 3627 & 4640 & 9432 & 63 \\
\hline $\mathrm{H}(18 \mathrm{~A})$ & 3804 & 3756 & 7602 & 46 \\
\hline $\mathrm{H}(18 \mathrm{~B})$ & 4039 & 2990 & 7639 & 46 \\
\hline $\mathrm{H}(18 \mathrm{C})$ & 4459 & 3627 & 7738 & 46 \\
\hline $\mathrm{H}(21 \mathrm{~A})$ & 7986 & 2419 & 10886 & 43 \\
\hline $\mathrm{H}(23 \mathrm{~A})$ & 8174 & 1077 & 9749 & 43 \\
\hline $\mathrm{H}(25 \mathrm{~A})$ & 7147 & 3005 & 10715 & 57 \\
\hline $\mathrm{H}(25 \mathrm{~B})$ & 6592 & 2579 & 10352 & 57 \\
\hline $\mathrm{H}(25 \mathrm{C})$ & 6809 & 3173 & 10040 & 57 \\
\hline $\mathrm{H}(26 \mathrm{~A})$ & 8960 & 1278 & 10596 & 100 \\
\hline $\mathrm{H}(26 \mathrm{~B})$ & 8803 & 1420 & 11155 & 100 \\
\hline $\mathrm{H}(26 \mathrm{C})$ & 8999 & 2039 & 10849 & 100 \\
\hline
\end{tabular}




\begin{tabular}{|c|c|c|c|c|}
\hline $\mathrm{H}(27 \mathrm{~A})$ & 7418 & 785 & 8877 & 59 \\
\hline $\mathrm{H}(27 \mathrm{~B})$ & 7010 & 1420 & 8599 & 59 \\
\hline $\mathrm{H}(27 \mathrm{C})$ & 6811 & 824 & 8924 & 59 \\
\hline $\mathrm{H}(30 \mathrm{~A})$ & 2804 & -874 & 7702 & 31 \\
\hline $\mathrm{H}(32 \mathrm{~A})$ & 4228 & -1508 & 8948 & 30 \\
\hline $\mathrm{H}(34 \mathrm{~A})$ & 2762 & 102 & 7128 & 40 \\
\hline $\mathrm{H}(34 \mathrm{~B})$ & 3327 & 217 & 7005 & 40 \\
\hline $\mathrm{H}(34 \mathrm{C})$ & 3190 & 700 & 7458 & 40 \\
\hline $\mathrm{H}(35 \mathrm{~A})$ & 2760 & -1874 & 8214 & 57 \\
\hline $\mathrm{H}(35 \mathrm{~B})$ & 3197 & -1891 & 8876 & 57 \\
\hline $\mathrm{H}(35 \mathrm{C})$ & 3326 & -2316 & 8386 & 57 \\
\hline $\mathrm{H}(5 \mathrm{~K})$ & $5700(20)$ & $860(20)$ & 9364(19) & 28 \\
\hline $\mathrm{H}(5 \mathrm{~J})$ & $5880(20)$ & $1450(20)$ & $9140(20)$ & 28 \\
\hline $\mathrm{H}(1 \mathrm{~A})$ & 6172 & 3685 & 9140 & 30 \\
\hline $\mathrm{H}(1 \mathrm{~B})$ & 5832 & 3749 & 9530 & 30 \\
\hline $\mathrm{H}(2 \mathrm{~A})$ & 5486 & 3672 & 8007 & 36 \\
\hline $\mathrm{H}(2 \mathrm{~B})$ & 5750 & 4074 & 7645 & 36 \\
\hline $\mathrm{H}(36 \mathrm{~A})$ & 5090 & -959 & 9150 & 44 \\
\hline $\mathrm{H}(36 \mathrm{~B})$ & 5046 & -146 & 9063 & 44 \\
\hline $\mathrm{H}(36 \mathrm{C})$ & 5148 & -622 & 8585 & 44 \\
\hline $\mathrm{H}(37 \mathrm{~A})$ & 5645 & 4871 & 9125 & 37 \\
\hline $\mathrm{H}(37 \mathrm{~B})$ & 5187 & 4346 & 8714 & 37 \\
\hline $\mathrm{H}(3 \mathrm{~A})$ & 6859 & 2954 & 8961 & 30 \\
\hline $\mathrm{H}(3 \mathrm{C})$ & 7049 & 3665 & 9213 & 30 \\
\hline $\mathrm{H}(4 \mathrm{~A})$ & 6338 & 2969 & 7862 & 36 \\
\hline $\mathrm{H}(4 \mathrm{~B})$ & 6485 & 2344 & 7577 & 36 \\
\hline $\mathrm{H}(38 \mathrm{~A})$ & 5601 & 5040 & 8181 & 39 \\
\hline $\mathrm{H}(38 \mathrm{~B})$ & 6200 & 4699 & 8542 & 39 \\
\hline $\mathrm{H}(39 \mathrm{~A})$ & 7697 & 3544 & 8724 & 30 \\
\hline $\mathrm{H}(39 \mathrm{~B})$ & 7115 & 3862 & 8283 & 30 \\
\hline $\mathrm{H}(5 \mathrm{~A})$ & 4053 & 2056 & 8433 & 32 \\
\hline $\mathrm{H}(5 \mathrm{~B})$ & 3500 & 1895 & 7932 & 32 \\
\hline $\mathrm{H}(6 \mathrm{~A})$ & 4518 & 904 & 8925 & 31 \\
\hline $\mathrm{H}(6 \mathrm{~B})$ & 4823 & 1015 & 9578 & 31 \\
\hline $\mathrm{H}(40 \mathrm{~A})$ & 7387 & 2896 & 7859 & 33 \\
\hline $\mathrm{H}(40 \mathrm{~B})$ & 7371 & 2424 & 8383 & 33 \\
\hline
\end{tabular}




\begin{tabular}{|c|c|c|c|c|}
\hline $\mathrm{H}(41 \mathrm{~A})$ & 3493 & 922 & 8529 & 31 \\
\hline $\mathrm{H}(41 \mathrm{~B})$ & 3201 & 1563 & 8710 & 31 \\
\hline $\mathrm{H}(42 \mathrm{~A})$ & 4101 & 1862 & 9435 & 31 \\
\hline $\mathrm{H}(42 \mathrm{~B})$ & 3881 & 1131 & 9559 & 31 \\
\hline $\mathrm{H}(48 \mathrm{~A})$ & 10644 & 4662 & 6597 & 42 \\
\hline $\mathrm{H}(50 \mathrm{~A})$ & 11386 & 6459 & 7172 & 41 \\
\hline $\mathrm{H}(52 \mathrm{~A})$ & 9365 & 5043 & 6803 & 52 \\
\hline $\mathrm{H}(52 \mathrm{~B})$ & 9795 & 4410 & 6978 & 52 \\
\hline $\mathrm{H}(52 \mathrm{C})$ & 9533 & 4664 & 6316 & 52 \\
\hline $\mathrm{H}(53 \mathrm{~A})$ & 11536 & 4816 & 6574 & 100 \\
\hline $\mathrm{H}(53 \mathrm{~B})$ & 11942 & 5285 & 7091 & 100 \\
\hline $\mathrm{H}(53 \mathrm{C})$ & 11667 & 5588 & 6445 & 100 \\
\hline $\mathrm{H}(54 \mathrm{~A})$ & 10960 & 7313 & 7523 & 55 \\
\hline $\mathrm{H}(54 \mathrm{~B})$ & 10543 & 7032 & 7820 & 55 \\
\hline $\mathrm{H}(54 \mathrm{C})$ & 10292 & 7366 & 7183 & 55 \\
\hline $\mathrm{H}(57 \mathrm{~A})$ & 7589 & 7839 & 8069 & 38 \\
\hline $\mathrm{H}(59 \mathrm{~A})$ & 9150 & 8433 & 9058 & 33 \\
\hline $\mathrm{H}(61 \mathrm{~A})$ & 7388 & 7084 & 7289 & 47 \\
\hline $\mathrm{H}(61 \mathrm{~B})$ & 7866 & 6519 & 7379 & 47 \\
\hline $\mathrm{H}(61 \mathrm{C})$ & 7807 & 7149 & 6945 & 47 \\
\hline $\mathrm{H}(62 \mathrm{~A})$ & 8417 & 8857 & 9299 & 78 \\
\hline $\mathrm{H}(62 \mathrm{~B})$ & 7906 & 8328 & 9174 & 78 \\
\hline $\mathrm{H}(62 \mathrm{C})$ & 7838 & 8977 & 8757 & 78 \\
\hline $\mathrm{H}(63 \mathrm{~A})$ & 9836 & 7458 & 8290 & 49 \\
\hline $\mathrm{H}(63 \mathrm{~B})$ & 9934 & 7593 & 8960 & 49 \\
\hline $\mathrm{H}(63 \mathrm{C})$ & 9882 & 8226 & 8531 & 49 \\
\hline $\mathrm{H}(66 \mathrm{~A})$ & 7471 & 5288 & 4541 & 27 \\
\hline $\mathrm{H}(68 \mathrm{~A})$ & 6525 & 6865 & 4692 & 29 \\
\hline $\mathrm{H}(70 \mathrm{~A})$ & 8672 & 5702 & 5824 & 42 \\
\hline $\mathrm{H}(70 \mathrm{~B})$ & 8583 & 5551 & 5159 & 42 \\
\hline $\mathrm{H}(70 \mathrm{C})$ & 8353 & 5020 & 5512 & 42 \\
\hline $\mathrm{H}(71 \mathrm{~A})$ & 6571 & 5423 & 3805 & 61 \\
\hline $\mathrm{H}(71 \mathrm{~B})$ & 6380 & 6207 & 3680 & 61 \\
\hline $\mathrm{H}(71 \mathrm{C})$ & 6113 & 5730 & 4037 & 61 \\
\hline $\mathrm{H}(72 \mathrm{~A})$ & 6843 & 7668 & 5439 & 45 \\
\hline $\mathrm{H}(72 \mathrm{~B})$ & 7509 & 7800 & 5675 & 45 \\
\hline
\end{tabular}




\begin{tabular}{|c|c|c|c|c|}
\hline $\mathrm{H}(72 \mathrm{C})$ & 7243 & 7353 & 6052 & 45 \\
\hline $\mathrm{H}(75 \mathrm{~A})$ & 9621 & 3434 & 9688 & 39 \\
\hline $\mathrm{H}(77 \mathrm{~A})$ & 8492 & 2855 & 8142 & 38 \\
\hline $\mathrm{H}(79 \mathrm{~A})$ & 10232 & 4361 & 9788 & 51 \\
\hline $\mathrm{H}(79 \mathrm{~B})$ & 9962 & 4970 & 9335 & 51 \\
\hline $\mathrm{H}(79 \mathrm{C})$ & 10410 & 4462 & 9237 & 51 \\
\hline $\mathrm{H}(80 \mathrm{~B})$ & 9086 & 2454 & 9683 & 76 \\
\hline $\mathrm{H}(80 \mathrm{~A})$ & 8924 & 2014 & 9096 & 76 \\
\hline $\mathrm{H}(80 \mathrm{C})$ & 8458 & 2480 & 9206 & 76 \\
\hline $\mathrm{H}(81 \mathrm{~A})$ & 8368 & 3433 & 7277 & 50 \\
\hline $\mathrm{H}(81 \mathrm{~B})$ & 8953 & 3715 & 7267 & 50 \\
\hline $\mathrm{H}(81 \mathrm{C})$ & 8505 & 4236 & 7351 & 50 \\
\hline $\mathrm{H}(10 \mathrm{~J})$ & $8190(20)$ & $5860(20)$ & $6660(20)$ & 32 \\
\hline $\mathrm{H}(10 \mathrm{~K})$ & $8160(20)$ & $5290(30)$ & $6650(20)$ & 32 \\
\hline $\mathrm{H}(7 \mathrm{D})$ & 8482 & 8126 & 6468 & 31 \\
\hline $\mathrm{H}(7 \mathrm{E})$ & 8090 & 8174 & 6803 & 31 \\
\hline $\mathrm{H}(8 \mathrm{D})$ & 9540 & 8114 & 7187 & 46 \\
\hline $\mathrm{H}(8 \mathrm{E})$ & 9903 & 8511 & 6927 & 46 \\
\hline $\mathrm{H}(82 \mathrm{~A})$ & 8866 & 8787 & 7494 & 40 \\
\hline $\mathrm{H}(82 \mathrm{~B})$ & 8470 & 9310 & 7017 & 40 \\
\hline $\mathrm{H}(83 \mathrm{~A})$ & 9049 & 9153 & 6456 & 38 \\
\hline $\mathrm{H}(83 \mathrm{~B})$ & 9395 & 9482 & 7082 & 38 \\
\hline $\mathrm{H}(9 \mathrm{D})$ & 8632 & 7484 & 5765 & 33 \\
\hline $\mathrm{H}(9 \mathrm{E})$ & 8384 & 8171 & 5496 & 33 \\
\hline $\mathrm{H}(10 \mathrm{E})$ & 9681 & 7462 & 6218 & 43 \\
\hline $\mathrm{H}(10 \mathrm{~F})$ & 9944 & 6811 & 6089 & 43 \\
\hline $\mathrm{H}(84 \mathrm{~A})$ & 9235 & 8261 & 5333 & 37 \\
\hline $\mathrm{H}(84 \mathrm{~B})$ & 8773 & 7870 & 4798 & 37 \\
\hline $\mathrm{H}(85 \mathrm{~A})$ & 9128 & 6818 & 5208 & 48 \\
\hline $\mathrm{H}(85 \mathrm{~B})$ & 9616 & 7265 & 5122 & 48 \\
\hline $\mathrm{H}(11 \mathrm{~A})$ & 9108 & 6332 & 8547 & 37 \\
\hline $\mathrm{H}(11 \mathrm{~B})$ & 9568 & 6234 & 9147 & 37 \\
\hline $\mathrm{H}(12 \mathrm{~B})$ & 8678 & 5188 & 8100 & 41 \\
\hline $\mathrm{H}(12 \mathrm{C})$ & 8045 & 5253 & 7765 & 41 \\
\hline $\mathrm{H}(86 \mathrm{~A})$ & 8792 & 5936 & 9416 & 40 \\
\hline $\mathrm{H}(86 \mathrm{~B})$ & 9011 & 5269 & 9185 & 40 \\
\hline
\end{tabular}




\begin{tabular}{|c|c|c|c|c|}
\hline $\mathrm{H}(87 \mathrm{~A})$ & 8026 & 5399 & 8718 & 46 \\
\hline $\mathrm{H}(87 \mathrm{~B})$ & 8122 & 6127 & 8469 & 46 \\
\hline $\mathrm{H}(92 \mathrm{~A})$ & 4155 & 1189 & 6959 & 32 \\
\hline $\mathrm{H}(92 \mathrm{~B})$ & 4296 & 1729 & 7438 & 32 \\
\hline $\mathrm{H}(93 \mathrm{~A})$ & 2617 & 2398 & 5967 & 95 \\
\hline $\mathrm{H}(93 \mathrm{~B})$ & 2488 & 1875 & 5477 & 95 \\
\hline $\mathrm{H}(43 \mathrm{~A})$ & 3701 & 2438 & 6671 & 40 \\
\hline $\mathrm{H}(43 \mathrm{~B})$ & 3329 & 2032 & 6962 & 40 \\
\hline $\mathrm{H}(44 \mathrm{~A})$ & 3477 & 1640 & 5918 & 71 \\
\hline $\mathrm{H}(44 \mathrm{~B})$ & 3145 & 1180 & 6220 & 71 \\
\hline $\mathrm{H}(3 \mathrm{SA})$ & 10552 & 5513 & 8337 & 43 \\
\hline $\mathrm{H}(3 \mathrm{SB})$ & 10025 & 5927 & 8267 & 43 \\
\hline $\mathrm{H}(4 \mathrm{SA})$ & 11402 & 6616 & 9959 & 81 \\
\hline $\mathrm{H}(4 \mathrm{SB})$ & 11946 & 6228 & 10047 & 81 \\
\hline $\mathrm{H}(3 \mathrm{SC})$ & 10515 & 6134 & 9299 & 34 \\
\hline $\mathrm{H}(3 \mathrm{SD})$ & 10697 & 6703 & 8935 & 34 \\
\hline $\mathrm{H}(4 \mathrm{SC})$ & 11306 & 5446 & 9334 & 51 \\
\hline $\mathrm{H}(4 \mathrm{SD})$ & 11496 & 6043 & 8998 & 51 \\
\hline $\mathrm{H}(3 \mathrm{SE})$ & 10116 & 5773 & 8605 & 43 \\
\hline $\mathrm{H}(3 \mathrm{SF})$ & 10311 & 5751 & 8093 & 43 \\
\hline $\mathrm{H}(4 \mathrm{SE})$ & 11852 & 6334 & 9687 & 81 \\
\hline $\mathrm{H}(4 \mathrm{SF})$ & 11679 & 6385 & 10212 & 81 \\
\hline $\mathrm{H}(3 \mathrm{SG})$ & 11258 & 5726 & 8877 & 52 \\
\hline $\mathrm{H}(3 \mathrm{SH})$ & 11066 & 6511 & 8726 & 52 \\
\hline $\mathrm{H}(4 \mathrm{SG})$ & 10808 & 5725 & 9547 & 57 \\
\hline $\mathrm{H}(4 \mathrm{SH})$ & 10690 & 6534 & 9425 & 57 \\
\hline $\mathrm{H}(5 \mathrm{SC})$ & 7490 & 10589 & 5281 & 65 \\
\hline $\mathrm{H}(5 \mathrm{SD})$ & 6957 & 10765 & 4760 & 65 \\
\hline $\mathrm{H}(6 \mathrm{SC})$ & 7542 & 9285 & 5591 & 55 \\
\hline $\mathrm{H}(6 \mathrm{SD})$ & 8121 & 8960 & 5739 & 55 \\
\hline $\mathrm{H}(5 \mathrm{SA})$ & 7360 & 10046 & 4254 & 90 \\
\hline $\mathrm{H}(5 \mathrm{SB})$ & 6995 & 9599 & 4527 & 90 \\
\hline $\mathrm{H}(6 \mathrm{SA})$ & 7913 & 9095 & 4743 & 82 \\
\hline $\mathrm{H}(6 \mathrm{SB})$ & 8204 & 9776 & 5085 & 82 \\
\hline $\mathrm{H}(7 \mathrm{SC})$ & 7925 & 896 & 6487 & 85 \\
\hline $\mathrm{H}(7 \mathrm{SD})$ & 7732 & 176 & 6260 & 85 \\
\hline
\end{tabular}




\begin{tabular}{lrrrr} 
H(8SC) & 6627 & 935 & 7255 & 75 \\
$\mathrm{H}(8 \mathrm{SD})$ & 6821 & 1661 & 7463 & 75 \\
$\mathrm{H}(7 \mathrm{SB})$ & 7316 & 196 & 6973 & 66 \\
$\mathrm{H}(7 \mathrm{SA})$ & 6887 & 512 & 6375 & 66 \\
$\mathrm{H}(8 \mathrm{SB})$ & 7635 & 1330 & 7305 & 49 \\
$\mathrm{H}(8 \mathrm{SA})$ & 7179 & 1631 & 6717 & 49 \\
$\mathrm{H}(9 \mathrm{SA})$ & 7695 & 4184 & 5928 & 40 \\
$\mathrm{H}(9 \mathrm{SB})$ & 7877 & 4759 & 6377 & 40 \\
$\mathrm{H}(10 \mathrm{~A})$ & 5966 & 5044 & 5285 & 46 \\
$\mathrm{H}(10 B)$ & 5800 & 4481 & 4825 & 46 \\
$\mathrm{H}(9 \mathrm{SC})$ & 7069 & 5321 & 5714 & 26 \\
$\mathrm{H}(9 \mathrm{SD})$ & 6894 & 4863 & 6155 & 26 \\
$\mathrm{H}(10 \mathrm{C})$ & 6782 & 4461 & 5011 & 28 \\
$\mathrm{H}(10 \mathrm{D})$ & 6649 & 3960 & 5454 & 28 \\
$\mathrm{H}(11 \mathrm{~J})$ & -576 & 790 & 9610 & 171 \\
$\mathrm{H}(11 \mathrm{I})$ & -100 & 1228 & 9565 & 171 \\
$\mathrm{H}(11 \mathrm{H})$ & 470 & 473 & 10300 & 112 \\
$\mathrm{H}(11 \mathrm{G})$ & 361 & 154 & 9668 & 112 \\
& & & & \\
\hline
\end{tabular}


Table for Compound 6: Mg(pmdta)(OMes) 
Table 1. Crystal data and structure refinement for compound 6.

Identification code

Empirical formula

Formula weight

Crystal system

Space group

Unit cell dimensions

Volume

Z

Density (calculated)

Absorption coefficient

Crystal size

$\mathrm{F}(000)$ wt015

$\mathrm{C}_{27} \mathrm{H}_{45} \mathrm{Mg} \mathrm{N}_{3} \mathrm{O}_{2}$

467.97

Monoclinic

$\mathrm{P} 2(1) / \mathrm{c}$

$\mathrm{a}=12.7034(6) \AA$

$\alpha=90^{\circ}$

$\mathrm{b}=13.6576(7) \AA$

$\beta=96.199(1)^{\circ}$.

$\mathrm{c}=15.6115(8) \AA$

$\gamma=90^{\circ}$.

2692.7(2) $\AA^{3}$

4

$1.154 \mathrm{~g} / \mathrm{cm}^{3}$

$0.093 \mathrm{~mm}^{-1}$

$0.70 \times 0.45 \times 0.36 \mathrm{~mm}^{3}$

1024 


\section{Data Collection:}

Diffractometer

Theta range for data collection

Index ranges

Scan Type

Temperature

Wavelength

Reflections collected

Independent reflections

Completeness to theta $=28.29^{\circ}$

Absorption correction

Max. and min. transmission
CCD area detector

1.99 to $28.29^{\circ}$.

$-16<=\mathrm{h}<=16,-18<=\mathrm{k}<=18,-20<=\mathrm{l}<=20$

phi and omega scans

95(2) K

$0.71073 \AA$

42124

$6683[\mathrm{R}(\mathrm{int})=0.0381]$

$99.9 \%$

Semi-empirical from equivalents

0.9672 and 0.9376

\section{Solution and Refinement:}

Solution

Refinement method

Data / restraints / parameters

Goodness-of-fit on $\mathrm{F}^{2}$

Final $R$ indices [I $>2 \operatorname{sigma}(\mathrm{I})]$

$\mathrm{R}$ indices (all data)

Largest diff. peak and hole direct methods

Full-matrix least-squares on $\mathrm{F}^{2}$

$6683 / 0 / 316$

1.035

$\mathrm{R} 1=0.0414, \mathrm{wR} 2=0.1047$

$\mathrm{R} 1=0.0512, \mathrm{wR} 2=0.1107$

0.484 and -0.382 e. $\AA^{-3}$ 
Table 2. Atomic coordinates ( $\left.x 1^{4}\right)$ and equivalent isotropic displacement parameters $\left(\AA^{2} \times 10^{3}\right)$ for compound $6 . \mathrm{U}(\mathrm{eq})$ is defined as one third of the trace of the orthogonalized $U^{i j}$ tensor.

\begin{tabular}{|c|c|c|c|c|}
\hline & $\mathrm{x}$ & $\mathrm{y}$ & $\mathrm{z}$ & $\mathrm{U}(\mathrm{eq})$ \\
\hline $\operatorname{Mg}(1)$ & $3399(1)$ & $600(1)$ & $2166(1)$ & $14(1)$ \\
\hline $\mathrm{O}(1)$ & $4810(1)$ & 1127(1) & $2454(1)$ & $18(1)$ \\
\hline $\mathrm{C}(1)$ & $5725(1)$ & $1296(1)$ & $2146(1)$ & $16(1)$ \\
\hline$C(2)$ & $6689(1)$ & $945(1)$ & 2592(1) & $18(1)$ \\
\hline$C(3)$ & $7643(1)$ & 1090(1) & $2247(1)$ & $21(1)$ \\
\hline$C(4)$ & $7712(1)$ & 1606(1) & $1486(1)$ & $21(1)$ \\
\hline$C(5)$ & $6773(1)$ & 1991(1) & 1078(1) & 19(1) \\
\hline$C(6)$ & 5791(1) & 1852(1) & $1386(1)$ & $17(1)$ \\
\hline$C(7)$ & $6669(1)$ & $446(1)$ & $3452(1)$ & $24(1)$ \\
\hline $\mathrm{C}(8)$ & $8757(1)$ & 1769(1) & 1133(1) & $30(1)$ \\
\hline $\mathrm{C}(9)$ & $4807(1)$ & $2300(1)$ & $919(1)$ & $20(1)$ \\
\hline $\mathrm{O}(2)$ & $2623(1)$ & 1322(1) & $1280(1)$ & $23(1)$ \\
\hline $\mathrm{C}(10)$ & 1959(1) & 1916(1) & $828(1)$ & $18(1)$ \\
\hline $\mathrm{C}(11)$ & 1581(1) & 2779(1) & 1194(1) & $20(1)$ \\
\hline$C(12)$ & $838(1)$ & $3366(1)$ & $716(1)$ & $22(1)$ \\
\hline$C(13)$ & $434(1)$ & $3136(1)$ & $-122(1)$ & $22(1)$ \\
\hline$C(14)$ & $838(1)$ & 2309(1) & $-490(1)$ & $21(1)$ \\
\hline$C(15)$ & $1597(1)$ & 1709(1) & $-44(1)$ & $20(1)$ \\
\hline$C(16)$ & 1969(1) & 3032(1) & 2113(1) & $31(1)$ \\
\hline$C(17)$ & $-434(1)$ & $3742(1)$ & $-607(1)$ & $32(1)$ \\
\hline$C(18)$ & $2043(1)$ & $850(1)$ & $-488(1)$ & $29(1)$ \\
\hline $\mathrm{N}(1)$ & $3916(1)$ & $-769(1)$ & $1565(1)$ & $22(1)$ \\
\hline $\mathrm{N}(2)$ & 1935(1) & $-471(1)$ & $2267(1)$ & $26(1)$ \\
\hline $\mathrm{N}(3)$ & $3138(1)$ & $653(1)$ & $3570(1)$ & $22(1)$ \\
\hline$C(19)$ & $2956(1)$ & $-1355(1)$ & $1256(1)$ & $22(1)$ \\
\hline $\mathrm{C}(20)$ & $2265(2)$ & $-1470(1)$ & 1981(1) & $23(1)$ \\
\hline $\mathrm{C}\left(19^{\prime}\right)$ & $3142(4)$ & $-1606(3)$ & $1765(4)$ & $19(1)$ \\
\hline $\mathrm{C}\left(20^{\prime}\right)$ & 2021(4) & $-1223(4)$ & $1590(4)$ & $22(1)$ \\
\hline $\mathrm{C}(21)$ & 1743(1) & $-570(1)$ & $3176(1)$ & $30(1)$ \\
\hline $\mathrm{C}(22)$ & 2031(1) & $368(1)$ & $3655(1)$ & $29(1)$ \\
\hline$C(23)$ & $4368(2)$ & $-500(1)$ & 764(1) & $38(1)$ \\
\hline
\end{tabular}


C(24)

$\mathrm{C}(25)$

C(26)

C(27)
4743(1)

986(1)

3879(1)

3339(1)
$-1286(1)$

$-62(1)$

$-40(1)$

1635(1)
2108(1)

1775(1)

4047(1)

3953(1)
38(1)

38(1)

32(1)

30(1) 
Table 3. Bond lengths [̊̊] for compound 6.

\begin{tabular}{llll}
\hline $\mathrm{Mg}(1)-\mathrm{O}(2)$ & $1.8880(9)$ & $\mathrm{C}(12)-\mathrm{C}(13)$ & $1.3886(19)$ \\
$\mathrm{Mg}(1)-\mathrm{O}(1)$ & $1.9386(9)$ & $\mathrm{C}(13)-\mathrm{C}(14)$ & $1.3902(19)$ \\
$\mathrm{Mg}(1)-\mathrm{N}(1)$ & $2.2228(11)$ & $\mathrm{C}(13)-\mathrm{C}(17)$ & $1.5148(17)$ \\
$\mathrm{Mg}(1)-\mathrm{N}(3)$ & $2.2523(11)$ & $\mathrm{C}(14)-\mathrm{C}(15)$ & $1.3934(17)$ \\
$\mathrm{Mg}(1)-\mathrm{N}(2)$ & $2.3851(12)$ & $\mathrm{C}(15)-\mathrm{C}(18)$ & $1.5038(18)$ \\
$\mathrm{O}(1)-\mathrm{C}(1)$ & $1.3246(14)$ & $\mathrm{N}(1)-\mathrm{C}(24)$ & $1.4589(18)$ \\
$\mathrm{C}(1)-\mathrm{C}(6)$ & $1.4180(16)$ & $\mathrm{N}(1)-\mathrm{C}(23)$ & $1.4761(19)$ \\
$\mathrm{C}(1)-\mathrm{C}(2)$ & $\mathrm{N}(1)-\mathrm{C}(19)$ & $1.495(2)$ \\
$\mathrm{C}(2)-\mathrm{C}(3)$ & $1.4252(16)$ & $\left.\mathrm{N}(1)-\mathrm{C}(19)^{\prime}\right)$ & $1.561(5)$ \\
$\mathrm{C}(2)-\mathrm{C}(7)$ & $1.3919(17)$ & $\mathrm{N}(2)-\mathrm{C}(25)$ & $1.467(2)$ \\
$\mathrm{C}(3)-\mathrm{C}(4)$ & $1.5084(17)$ & $\mathrm{N}(2)-\mathrm{C}(21)$ & $1.4705(19)$ \\
$\mathrm{C}(4)-\mathrm{C}(5)$ & $1.3921(18)$ & $\mathrm{N}(2)-\mathrm{C}\left(20^{\prime}\right)$ & $1.487(6)$ \\
$\mathrm{C}(4)-\mathrm{C}(8)$ & $1.3938(18)$ & $\mathrm{N}(2)-\mathrm{C}(20)$ & $1.510(2)$ \\
$\mathrm{C}(5)-\mathrm{C}(6)$ & $1.5082(17)$ & $\mathrm{N}(3)-\mathrm{C}(22)$ & $1.4786(17)$ \\
$\mathrm{C}(6)-\mathrm{C}(9)$ & $1.3972(17)$ & $\mathrm{N}(3)-\mathrm{C}(26)$ & $1.4789(17)$ \\
$\mathrm{O}(2)-\mathrm{C}(10)$ & $1.5072(16)$ & $\mathrm{N}(3)-\mathrm{C}(27)$ & $1.4799(18)$ \\
$\mathrm{C}(10)-\mathrm{C}(11)$ & $1.3193(14)$ & $\mathrm{C}(19)-\mathrm{C}(20)$ & $1.513(3)$ \\
$\mathrm{C}(10)-\mathrm{C}(15)$ & $1.4155(17)$ & $\left.\mathrm{C}(19)^{\prime}\right)-\mathrm{C}\left(20^{\prime}\right)$ & $1.514(8)$ \\
$\mathrm{C}(11)-\mathrm{C}(12)$ & $1.4181(17)$ & $\mathrm{C}(21)-\mathrm{C}(22)$ & $1.509(2)$ \\
$\mathrm{C}(11)-\mathrm{C}(16)$ & $1.3924(17)$ & & \\
& $1.5055(18)$ & & \\
\hline
\end{tabular}


Table 4. Bond angles $\left[{ }^{\circ}\right]$ for compound 6.

\begin{tabular}{|c|c|c|c|}
\hline $\mathrm{O}(2)-\mathrm{Mg}(1)-\mathrm{O}(1)$ & 111.91(4) & $\mathrm{C}(12)-\mathrm{C}(13)-\mathrm{C}(17)$ & $121.46(12)$ \\
\hline $\mathrm{O}(2)-\mathrm{Mg}(1)-\mathrm{N}(1)$ & $106.52(5)$ & $\mathrm{C}(14)-\mathrm{C}(13)-\mathrm{C}(17)$ & $121.28(12)$ \\
\hline $\mathrm{O}(1)-\mathrm{Mg}(1)-\mathrm{N}(1)$ & $95.87(4)$ & $C(13)-C(14)-C(15)$ & $122.53(12)$ \\
\hline $\mathrm{O}(2)-\mathrm{Mg}(1)-\mathrm{N}(3)$ & $125.15(4)$ & $C(14)-C(15)-C(10)$ & $119.82(11)$ \\
\hline $\mathrm{O}(1)-\mathrm{Mg}(1)-\mathrm{N}(3)$ & $89.57(4)$ & $C(14)-C(15)-C(18)$ & $120.21(11)$ \\
\hline $\mathrm{N}(1)-\mathrm{Mg}(1)-\mathrm{N}(3)$ & $121.28(4)$ & $C(10)-C(15)-C(18)$ & $119.96(11)$ \\
\hline $\mathrm{O}(2)-\mathrm{Mg}(1)-\mathrm{N}(2)$ & $91.07(4)$ & $\mathrm{C}(24)-\mathrm{N}(1)-\mathrm{C}(23)$ & $106.89(12)$ \\
\hline $\mathrm{O}(1)-\mathrm{Mg}(1)-\mathrm{N}(2)$ & $157.01(4)$ & $\mathrm{C}(24)-\mathrm{N}(1)-\mathrm{C}(19)$ & $116.11(13)$ \\
\hline $\mathrm{N}(1)-\mathrm{Mg}(1)-\mathrm{N}(2)$ & $77.33(4)$ & $\mathrm{C}(23)-\mathrm{N}(1)-\mathrm{C}(19)$ & $103.79(12)$ \\
\hline $\mathrm{N}(3)-\mathrm{Mg}(1)-\mathrm{N}(2)$ & $75.93(4)$ & $\mathrm{C}(24)-\mathrm{N}(1)-\mathrm{C}\left(19^{\prime}\right)$ & $87.7(2)$ \\
\hline $\mathrm{C}(1)-\mathrm{O}(1)-\mathrm{Mg}(1)$ & $143.68(8)$ & $\mathrm{C}(23)-\mathrm{N}(1)-\mathrm{C}\left(19^{\prime}\right)$ & $131.5(2)$ \\
\hline $\mathrm{O}(1)-\mathrm{C}(1)-\mathrm{C}(6)$ & $122.08(10)$ & $\mathrm{C}(19)-\mathrm{N}(1)-\mathrm{C}\left(19^{\prime}\right)$ & $33.15(19)$ \\
\hline $\mathrm{O}(1)-\mathrm{C}(1)-\mathrm{C}(2)$ & $120.31(10)$ & $\mathrm{C}(24)-\mathrm{N}(1)-\mathrm{Mg}(1)$ & $112.85(9)$ \\
\hline$C(6)-C(1)-C(2)$ & $117.58(11)$ & $\mathrm{C}(23)-\mathrm{N}(1)-\mathrm{Mg}(1)$ & $107.94(8)$ \\
\hline $\mathrm{C}(3)-\mathrm{C}(2)-\mathrm{C}(1)$ & $120.07(11)$ & $\mathrm{C}(19)-\mathrm{N}(1)-\mathrm{Mg}(1)$ & $108.58(9)$ \\
\hline $\mathrm{C}(3)-\mathrm{C}(2)-\mathrm{C}(7)$ & $120.49(11)$ & $\mathrm{C}\left(19^{\prime}\right)-\mathrm{N}(1)-\mathrm{Mg}(1)$ & $108.06(19)$ \\
\hline $\mathrm{C}(1)-\mathrm{C}(2)-\mathrm{C}(7)$ & $119.41(11)$ & $\mathrm{C}(25)-\mathrm{N}(2)-\mathrm{C}(21)$ & $109.17(11)$ \\
\hline $\mathrm{C}(2)-\mathrm{C}(3)-\mathrm{C}(4)$ & $122.69(11)$ & $\mathrm{C}(25)-\mathrm{N}(2)-\mathrm{C}\left(20^{\prime}\right)$ & $90.5(3)$ \\
\hline $\mathrm{C}(3)-\mathrm{C}(4)-\mathrm{C}(5)$ & $116.83(11)$ & $\mathrm{C}(21)-\mathrm{N}(2)-\mathrm{C}\left(20^{\prime}\right)$ & $130.9(3)$ \\
\hline $\mathrm{C}(3)-\mathrm{C}(4)-\mathrm{C}(8)$ & $121.74(12)$ & $\mathrm{C}(25)-\mathrm{N}(2)-\mathrm{C}(20)$ & $115.26(13)$ \\
\hline $\mathrm{C}(5)-\mathrm{C}(4)-\mathrm{C}(8)$ & $121.40(12)$ & $\mathrm{C}(21)-\mathrm{N}(2)-\mathrm{C}(20)$ & $106.43(13)$ \\
\hline$C(4)-C(5)-C(6)$ & $122.87(11)$ & $\mathrm{C}\left(20^{\prime}\right)-\mathrm{N}(2)-\mathrm{C}(20)$ & $28.4(2)$ \\
\hline$C(5)-C(6)-C(1)$ & $119.80(11)$ & $\mathrm{C}(25)-\mathrm{N}(2)-\mathrm{Mg}(1)$ & $109.55(9)$ \\
\hline $\mathrm{C}(5)-\mathrm{C}(6)-\mathrm{C}(9)$ & $120.16(11)$ & $\mathrm{C}(21)-\mathrm{N}(2)-\mathrm{Mg}(1)$ & $109.38(8)$ \\
\hline $\mathrm{C}(1)-\mathrm{C}(6)-\mathrm{C}(9)$ & $120.04(10)$ & $\mathrm{C}\left(20^{\prime}\right)-\mathrm{N}(2)-\mathrm{Mg}(1)$ & $105.0(2)$ \\
\hline $\mathrm{C}(10)-\mathrm{O}(2)-\mathrm{Mg}(1)$ & $165.17(9)$ & $\mathrm{C}(20)-\mathrm{N}(2)-\mathrm{Mg}(1)$ & 106.90(9) \\
\hline $\mathrm{O}(2)-\mathrm{C}(10)-\mathrm{C}(11)$ & $121.44(11)$ & $\mathrm{C}(22)-\mathrm{N}(3)-\mathrm{C}(26)$ & $110.29(11)$ \\
\hline $\mathrm{O}(2)-\mathrm{C}(10)-\mathrm{C}(15)$ & $120.84(11)$ & $\mathrm{C}(22)-\mathrm{N}(3)-\mathrm{C}(27)$ & $109.02(11)$ \\
\hline $\mathrm{C}(11)-\mathrm{C}(10)-\mathrm{C}(15)$ & $117.72(11)$ & $\mathrm{C}(26)-\mathrm{N}(3)-\mathrm{C}(27)$ & $107.61(11)$ \\
\hline $\mathrm{C}(12)-\mathrm{C}(11)-\mathrm{C}(10)$ & $120.16(11)$ & $\mathrm{C}(22)-\mathrm{N}(3)-\mathrm{Mg}(1)$ & $108.76(8)$ \\
\hline $\mathrm{C}(12)-\mathrm{C}(11)-\mathrm{C}(16)$ & $120.81(12)$ & $\mathrm{C}(26)-\mathrm{N}(3)-\mathrm{Mg}(1)$ & $108.49(8)$ \\
\hline $\mathrm{C}(10)-\mathrm{C}(11)-\mathrm{C}(16)$ & $119.00(11)$ & $\mathrm{C}(27)-\mathrm{N}(3)-\mathrm{Mg}(1)$ & $112.67(8)$ \\
\hline$C(13)-C(12)-C(11)$ & $122.36(12)$ & $\mathrm{N}(1)-\mathrm{C}(19)-\mathrm{C}(20)$ & $109.49(14)$ \\
\hline $\mathrm{C}(12)-\mathrm{C}(13)-\mathrm{C}(14)$ & $117.24(11)$ & $\mathrm{N}(2)-\mathrm{C}(20)-\mathrm{C}(19)$ & $109.25(14)$ \\
\hline
\end{tabular}


$\mathrm{C}\left(20^{\prime}\right)-\mathrm{C}\left(19^{\prime}\right)-\mathrm{N}(1)$

$\mathrm{N}(2)-\mathrm{C}\left(20^{\prime}\right)-\mathrm{C}\left(19^{\prime}\right)$
108.1(4)

104.5(4)
$\mathrm{N}(2)-\mathrm{C}(21)-\mathrm{C}(22)$

$\mathrm{N}(3)-\mathrm{C}(22)-\mathrm{C}(21)$
110.13(11)

111.08(11) 
Table 5. Anisotropic displacement parameters $\left(\AA^{2} \times 10^{3}\right)$ for compound 6. The anisotropic displacement factor exponent takes the form: $-2 \pi^{2}\left[h^{2} a * 2 U^{11}+\ldots+2 h k^{*} b^{*} U^{12}\right]$

\begin{tabular}{|c|c|c|c|c|c|c|}
\hline & $\mathrm{U}^{11}$ & $\mathrm{U}^{22}$ & $\mathrm{U}^{33}$ & $\mathrm{U}^{23}$ & $\mathrm{U}^{13}$ & $\mathrm{U}^{12}$ \\
\hline $\operatorname{Mg}(1)$ & $15(1)$ & $15(1)$ & $13(1)$ & $0(1)$ & $1(1)$ & $1(1)$ \\
\hline $\mathrm{O}(1)$ & $15(1)$ & $23(1)$ & $16(1)$ & $1(1)$ & $2(1)$ & $-1(1)$ \\
\hline$C(1)$ & $17(1)$ & $16(1)$ & $15(1)$ & $-3(1)$ & $1(1)$ & $-2(1)$ \\
\hline$C(2)$ & $18(1)$ & $18(1)$ & $18(1)$ & $-1(1)$ & $-1(1)$ & $-1(1)$ \\
\hline$C(3)$ & $16(1)$ & $19(1)$ & $26(1)$ & $-3(1)$ & $-2(1)$ & $-1(1)$ \\
\hline$C(4)$ & $19(1)$ & $20(1)$ & $25(1)$ & $-5(1)$ & $5(1)$ & $-4(1)$ \\
\hline$C(5)$ & $23(1)$ & $18(1)$ & $17(1)$ & $-3(1)$ & $3(1)$ & $-5(1)$ \\
\hline$C(6)$ & 19(1) & $16(1)$ & $15(1)$ & $-3(1)$ & $0(1)$ & $-2(1)$ \\
\hline$C(7)$ & $21(1)$ & $30(1)$ & 21(1) & $5(1)$ & $-3(1)$ & $-1(1)$ \\
\hline$C(8)$ & 21(1) & $32(1)$ & $40(1)$ & $-2(1)$ & $10(1)$ & $-6(1)$ \\
\hline$C(9)$ & $22(1)$ & $22(1)$ & $17(1)$ & $1(1)$ & $0(1)$ & $-1(1)$ \\
\hline $\mathrm{O}(2)$ & 19(1) & $24(1)$ & $26(1)$ & $9(1)$ & $-4(1)$ & $1(1)$ \\
\hline$C(10)$ & $14(1)$ & $18(1)$ & $20(1)$ & $5(1)$ & $0(1)$ & $-2(1)$ \\
\hline $\mathrm{C}(11)$ & $20(1)$ & 21(1) & $18(1)$ & $4(1)$ & $3(1)$ & $-3(1)$ \\
\hline$C(12)$ & 21(1) & $18(1)$ & $27(1)$ & $4(1)$ & $7(1)$ & $1(1)$ \\
\hline$C(13)$ & $15(1)$ & $22(1)$ & $28(1)$ & $10(1)$ & $1(1)$ & $0(1)$ \\
\hline$C(14)$ & $20(1)$ & $23(1)$ & $20(1)$ & $5(1)$ & $-3(1)$ & $-4(1)$ \\
\hline$C(15)$ & 19(1) & $18(1)$ & 21(1) & $2(1)$ & $1(1)$ & $-2(1)$ \\
\hline$C(16)$ & $42(1)$ & $31(1)$ & $20(1)$ & $-1(1)$ & $2(1)$ & $-3(1)$ \\
\hline$C(17)$ & $22(1)$ & $32(1)$ & $42(1)$ & $14(1)$ & $-3(1)$ & $5(1)$ \\
\hline$C(18)$ & $37(1)$ & $24(1)$ & $25(1)$ & $-1(1)$ & $2(1)$ & $5(1)$ \\
\hline $\mathrm{N}(1)$ & $20(1)$ & $17(1)$ & $30(1)$ & $-5(1)$ & $4(1)$ & $0(1)$ \\
\hline $\mathrm{N}(2)$ & $23(1)$ & $24(1)$ & $32(1)$ & $-8(1)$ & $10(1)$ & $-6(1)$ \\
\hline $\mathrm{N}(3)$ & $18(1)$ & $30(1)$ & $17(1)$ & 1(1) & $3(1)$ & $1(1)$ \\
\hline$C(19)$ & $28(1)$ & $18(1)$ & 21(1) & $-3(1)$ & $3(1)$ & $-3(1)$ \\
\hline$C(20)$ & $27(1)$ & $16(1)$ & $25(1)$ & $-1(1)$ & $3(1)$ & $-4(1)$ \\
\hline $\mathrm{C}\left(19^{\prime}\right)$ & $27(3)$ & $12(2)$ & $17(2)$ & $0(2)$ & $0(2)$ & $-4(2)$ \\
\hline $\mathrm{C}\left(20^{\prime}\right)$ & $24(3)$ & $14(2)$ & $25(3)$ & $0(2)$ & $-5(2)$ & $-3(2)$ \\
\hline$C(21)$ & $23(1)$ & $29(1)$ & $39(1)$ & 11(1) & $9(1)$ & $-3(1)$ \\
\hline$C(22)$ & $20(1)$ & $45(1)$ & $22(1)$ & $2(1)$ & $8(1)$ & $1(1)$ \\
\hline$C(23)$ & $64(1)$ & $32(1)$ & $18(1)$ & $-4(1)$ & $4(1)$ & $15(1)$ \\
\hline
\end{tabular}




\begin{tabular}{lllllll}
$\mathrm{C}(24)$ & $57(1)$ & $28(1)$ & $27(1)$ & $3(1)$ & $0(1)$ & $22(1)$ \\
$\mathrm{C}(25)$ & $25(1)$ & $53(1)$ & $34(1)$ & $6(1)$ & $-3(1)$ & $-18(1)$ \\
$\mathrm{C}(26)$ & $26(1)$ & $46(1)$ & $23(1)$ & $12(1)$ & $2(1)$ & $5(1)$ \\
$\mathrm{C}(27)$ & $31(1)$ & $39(1)$ & $21(1)$ & $-9(1)$ & $2(1)$ & $0(1)$ \\
\hline
\end{tabular}


Table 6. Hydrogen coordinates ( $\left.x 1^{4}\right)$ and isotropic displacement parameters $\left(\AA^{2} \times 10^{3}\right)$ for compound 6.

\begin{tabular}{|c|c|c|c|c|}
\hline & $\mathrm{x}$ & $\mathrm{y}$ & $\mathrm{z}$ & $\mathrm{U}(\mathrm{eq})$ \\
\hline $\mathrm{H}(3)$ & 8272 & 827 & 2543 & 25 \\
\hline $\mathrm{H}(5)$ & 6801 & 2365 & 568 & 23 \\
\hline $\mathrm{H}(7 \mathrm{~A})$ & 7393 & 271 & 3683 & 36 \\
\hline $\mathrm{H}(7 \mathrm{~B})$ & 6236 & -149 & 3378 & 36 \\
\hline $\mathrm{H}(7 \mathrm{C})$ & 6366 & 890 & 3852 & 36 \\
\hline $\mathrm{H}(8 \mathrm{~A})$ & 8651 & 2194 & 625 & 46 \\
\hline $\mathrm{H}(8 \mathrm{~B})$ & 9046 & 1139 & 970 & 46 \\
\hline $\mathrm{H}(8 \mathrm{C})$ & 9254 & 2081 & 1574 & 46 \\
\hline $\mathrm{H}(9 \mathrm{~A})$ & 5006 & 2762 & 484 & 30 \\
\hline $\mathrm{H}(9 \mathrm{~B})$ & 4413 & 2646 & 1332 & 30 \\
\hline $\mathrm{H}(9 \mathrm{C})$ & 4362 & 1782 & 637 & 30 \\
\hline $\mathrm{H}(12)$ & 599 & 3944 & 972 & 26 \\
\hline $\mathrm{H}(14)$ & 587 & 2147 & -1068 & 25 \\
\hline $\mathrm{H}(16 \mathrm{~A})$ & 1756 & 3702 & 2237 & 46 \\
\hline $\mathrm{H}(16 \mathrm{~B})$ & 2743 & 2981 & 2198 & 46 \\
\hline $\mathrm{H}(16 \mathrm{C})$ & 1661 & 2576 & 2502 & 46 \\
\hline $\mathrm{H}(17 \mathrm{~A})$ & -216 & 3939 & -1165 & 48 \\
\hline $\mathrm{H}(17 \mathrm{~B})$ & -563 & 4328 & -270 & 48 \\
\hline $\mathrm{H}(17 \mathrm{C})$ & -1085 & 3353 & -699 & 48 \\
\hline $\mathrm{H}(18 \mathrm{~A})$ & 1697 & 802 & -1079 & 43 \\
\hline $\mathrm{H}(18 \mathrm{~B})$ & 1916 & 248 & -174 & 43 \\
\hline $\mathrm{H}(18 \mathrm{C})$ & 2806 & 942 & -501 & 43 \\
\hline $\mathrm{H}(19 \mathrm{~A})$ & 3171 & -2008 & 1061 & 27 \\
\hline $\mathrm{H}(19 \mathrm{~B})$ & 2554 & -1020 & 762 & 27 \\
\hline $\mathrm{H}(20 \mathrm{~A})$ & 1630 & -1862 & 1781 & 27 \\
\hline $\mathrm{H}(20 \mathrm{~B})$ & 2661 & -1818 & 2470 & 27 \\
\hline $\mathrm{H}(19 \mathrm{C})$ & 3239 & -2181 & 1395 & 23 \\
\hline $\mathrm{H}(19 \mathrm{D})$ & 3286 & -1809 & 2375 & 23 \\
\hline $\mathrm{H}(20 \mathrm{C})$ & 1900 & -928 & 1008 & 26 \\
\hline $\mathrm{H}(20 \mathrm{D})$ & 1500 & -1755 & 1635 & 26 \\
\hline
\end{tabular}




\begin{tabular}{lrrrr}
$\mathrm{H}(21 \mathrm{~A})$ & 2173 & -1115 & 3444 & 36 \\
$\mathrm{H}(21 \mathrm{~B})$ & 987 & -723 & 3211 & 36 \\
$\mathrm{H}(22 \mathrm{~A})$ & 1549 & 898 & 3425 & 34 \\
$\mathrm{H}(22 \mathrm{~B})$ & 1940 & 280 & 4273 & 34 \\
$\mathrm{H}(23 \mathrm{~A})$ & 4984 & -75 & 904 & 57 \\
$\mathrm{H}(23 \mathrm{~B})$ & 3833 & -153 & 379 & 57 \\
$\mathrm{H}(23 \mathrm{C})$ & 4586 & -1095 & 479 & 57 \\
$\mathrm{H}(24 \mathrm{~A})$ & 4922 & -1893 & 1822 & 57 \\
$\mathrm{H}(24 \mathrm{~B})$ & 4490 & -1440 & 2663 & 57 \\
$\mathrm{H}(24 \mathrm{C})$ & 5374 & -870 & 2202 & 57 \\
$\mathrm{H}(25 \mathrm{~A})$ & 382 & -496 & 1826 & 57 \\
$\mathrm{H}(25 \mathrm{~B})$ & 1106 & -4 & 1167 & 57 \\
$\mathrm{H}(25 \mathrm{C})$ & 837 & 586 & 2002 & 57 \\
$\mathrm{H}(26 \mathrm{~A})$ & 4609 & 163 & 3994 & 47 \\
$\mathrm{H}(26 \mathrm{~B})$ & 3763 & -699 & 3808 & 47 \\
$\mathrm{H}(26 \mathrm{C})$ & 3758 & -43 & 4656 & 47 \\
$\mathrm{H}(27 \mathrm{~A})$ & 2850 & 2109 & 3655 & 45 \\
$\mathrm{H}(27 \mathrm{~B})$ & 4070 & 1830 & 3894 & 45 \\
$\mathrm{H}(27 \mathrm{C})$ & 3231 & 1615 & 4565 & 45 \\
\hline & & & & \\
\hline
\end{tabular}

